\title{
Deterministic Chaos in Digital Cryptography
}

\author{
by Nikolai V. Ptitsyn
}

\author{
Supervisors: \\ Professor G. Evans \\ Professor V. M. Chernenky ${ }^{\dagger}$ \\ submitted in partial fulfillment of the requirements \\ for the award of a $\mathrm{PhD}$ degree at \\ De Montfort University \\ The Gateway, Leicester LE1 9BH, United Kingdom \\ Science and Engineering Research Centre \\ Institute of Simulation Sciences \\ Faculty of Computer Science and Engineering \\ tDepartment of Information Processing and Management Systems \\ Faculty of Information and Control Systems \\ Moscow State Technical University (n/a N. E. Bauman), Russia
}




\section{Abstract}

This thesis studies the application of deterministic chaos to digital cryptography. Cryptographic systems such as pseudo-random generators (PRNG), block ciphers and hash functions are regarded as a dynamic system $\langle X, f\rangle$, where $X$ is a state space (i.e. message space) and $f: X \rightarrow X$ is an iterated function. In both chaos theory and cryptography, the object of study is a dynamic system that performs an iterative nonlinear transformation of information in an apparently unpredictable but deterministic manner. In terms of chaos theory, the sensitivity to the initial conditions together with the mixing property ensures cryptographic confusion (statistical independence) and diffusion (uniform propagation of plaintext and key randomness into cihertext).

This synergetic relationship between the properties of chaotic and cryptographic systems is considered at both the theoretical and practical levels: The theoretical background upon which this relationship is based, includes discussions on chaos, ergodicity, complexity, randomness, unpredictability and entropy.

Two approaches to the finite-state implementation of chaotic systems (i.e. pseudo-chaos) are considered: (i) floating-point approximation of continuous-state chaos; (ii) binary pseudo-chaos. An overview is given of chaotic systems underpinning cryptographic algorithms along with their strengths and weaknesses. Though all conventional cryposystems are considered binary pseudo-chaos, neither chaos, nor pseudo-chaos are sufficient to guarantee cryptographic strength and security.

A dynamic system is said to have an analytical solution $x_{n}=\Phi\left(x_{0}\right)$ 
if any trajectory point $x_{n}$ can be computed directly from the initial conditions $x_{0}$, without performing $n$ iterations. A chaotic system with an analytical solution may have a unpredictable multi-valued map $x_{n+1}=$ $f\left(x_{n}\right)$. Their floating-point approximation is studied in the context of pseudo-random generators.

A cryptographic software system E-Larm ${ }^{\mathrm{TM}}$ implementing a multistream pseudo-chaotic generator is described. Several pseudo-chaotic systems including the logistic map, sine map, tangent- and logarithmfeedback maps, sawteeth and tent maps are evaluated by means of floatingpoint computations. Two types of partitioning are used to extract pseudo-random from the floating-point state variable: (i) combining the last significant bits of the floating-point number (for nonlinear maps); and (ii) threshold partitioning (for piecewise linear maps). Multi-round iterations are produced to decrease the bit dependence and increase nonlinearity. Relationships between pseudo-chaotic systems are introduced to avoid short cycles (each system influences periodically the states of other systems used in the encryption session).

An evaluation of cryptographic properties of E-Larm is given using graphical plots such as state distributions, phase-space portraits, spectral density Fourier transform, approximated entropy (APEN), cycle length histogram, as well as a variety of statistical tests from the National Institute of Standards and Technology (NIST) suite. Though E-Larm passes all tests recommended by NIST, an approach based on the floating-point approximation of chaos is inefficient in terms of the quality/performance ratio (compared with existing PRNG algorithms). Also no solution is known to control short cycles.

In conclusion, the role of chaos theory in cryptography is identified; disadvantages of floating-point pseudo-chaos are emphasized although binary pseudo-chaos is considered useful for cryptographic applications.

Keywords: chaos, cryptography, synergics, pseudo-chaos, randomness, complexity, unpredictability. 


\section{Acknowledgments}

First of all, I would like to thank my earlier supervisor, Professor Jonathan Blackledge, representing the British side in the partnership program between De Montfort and Moscow State Technical Universities for his ongoing support that has always been helpful and enjoyable.

I am grateful to my Russian supervisor Professor Valery Chernenky at MSTU who provided both valuable criticism and fresh ideas.

I wish to thank my Professor Gwynne Evans for taking generously my supervising at the end of the project.

I would like to acknowledge the members of Science and Engineering Research Centre at De Montfort University, especially Dr Martin Tuner and Dr Allan Evans for inspiring discussions and advice.

I wish to kindly thank Professor Michael Kapranov and Alexei Tomashevski of the Moscow Power Engineering Institute for their extended feedback.

I am deeply grateful to my parents and family for the variety of their support. My grand farther, Professor at Moscow Power Engineering Institute, contributed to my work giving academic and technical directions.

Finally, this work would not have been possible without research grants provided by Durand Technology Limited. 


\section{Notation}

$\begin{array}{ll}X & \text { state space } \\ X_{1}, X_{2}, \cdots & \text { subsets of X } \\ f & \text { iterated function } \\ x_{0} & \text { initial condition } \\ p & \text { parameter of an iterated function } \\ f^{n} & \text { composition of } n \text { iterations of } f \\ x_{n} & \text { system state after } n \text { iterations, i.e. } x_{n}=f^{n}\left(x_{0}\right) \\ \phi\left(x_{0}\right) & \text { trajectory starting at } x_{0} \\ \Phi\left(x_{0}\right) & \text { analytical (exact) solution of a dynamic system } \\ s & \text { symbol } \\ \alpha_{n} & n \text {-symbol string } \\ K(\alpha) & \text { algorithmic (Kolomogorov) complexity of } \alpha \\ c(\alpha) & \text { symbolic complexity of } \alpha \\ \mathcal{C}\left(x_{0}\right) & \text { complexity of trajectory staring at } x_{0} \\ \operatorname{Pr}(\alpha) & \text { probability of occurrence of } \alpha \\ \operatorname{Pr}(\alpha \mid \beta) & \text { probability of } \alpha \text { provided } \beta \text { has been obtained } \\ H_{n} & \text { entropy of } \alpha_{n} \\ h_{n}=h_{n+1 \mid n} & \text { conditional entropy of }(n+1) \text {-th symbol in } \alpha_{n} \\ h, h_{S h} & \text { entropy of the source (generator) } \\ h_{K S} & \text { Kolmogorov-Sinai entropy } \\ & \end{array}$




\section{IMAGING SERVICES NORTH}

Boston Spa, Wetherby

West Yorkshire, LS23 7BQ

www.bl.uk

PAGE MISSING IN

ORIGINAL 


\title{
Glossary of Terms
}

\author{
Cryptography \\ PRNG Pseudo-Random Number Generator \\ LFSR Linear Feedback Shift Register \\ NFSR Nonlinear Feedback Shift Register \\ LCG Linear Congruent Generator \\ SHA1G Secure Hash Algorithm 1 Generator \\ S-Box Substitution Box \\ P-Box Permutation Box \\ PKI Public Key Infrastructure \\ Mathematics \& Chaos Theory \\ PDF Probability Density Function \\ CDF Cumulative Distribution Function \\ KS Kolmogorov-Sinai (entropy) \\ APEN Approximated Entropy \\ FFT Fast Fourier Transform \\ Complexity Theory \\ $P \quad$ Polynomial-time Computations \\ NP Nondeterministic Polynomial Time Computations \\ BPP Bounded-away-error Probabilistic Polynomial Time Computations \\ Computer Science \\ CA Cellular Automata \\ FPA Floating-point Arithmetic \\ BA Binary Arithmetic \\ CPU Central Processing \\ NaN Not-a-Number (infinity or incomputable value) \\ Organizations and Standards \\ NIST National Institute of Standards and Technologies \\ IEEE Institute of Electronic and Electrical Engineers \\ FIPS Federal Information Processing Standard
}




\section{Contents}

$\begin{array}{ll}\text { Abstract } & 1\end{array}$

$\begin{array}{lc}\text { Acknowledgments } & 3\end{array}$

$\begin{array}{ll}\text { Notation } & 4\end{array}$

$\begin{array}{lc}\text { Glossary of Terms } & 6\end{array}$

$\begin{array}{ll}\text { Contents } & 7\end{array}$

$\begin{array}{ll}\text { List of Figures } & 11\end{array}$

1 Introduction $\quad 14$

1.1 The Information Society . . . . . . . . . . . . . . . . 14

1.2 Cryptography, Cryptanalysis and Cryptology . . . . . . . . . . 15

1.3 The Synergetic Approach to Cryptography . . . . . . . . . . . . . 17

1.4 Deterministic Chaos . . . . . . . . . . . . . . . . . . . . . 19

1.5 History of Chaos-based Cryptography . . . . . . . . . . . . 21

1.6 Original Contribution . . . . . . . . . . . . . . 23

2 Preliminaries $\quad 24$

2.1 Cryptography . . . . . . . . . . . . . . . . . 24

2.1.1 What is Digital Information? . . . . . . . . . . . 24

2.1 .2 Cryptographic System . . . . . . . . . . . . . 25

2.1.3 Encryption and Decryption . . . . . . . . . . . 26

2.1.4 Pseudo-Random Number Generators . . . . . . . . . . . . . 32 
2.1.5 Confusion and Diffusion . . . . . . . . . . . . 32

2.1 .6 Cryptanalysis . . . . . . . . . . . . . . . 33

2.2 Chaotic Dynamics . . . . . . . . . . . . . . . . 35

2.2 .1 Dynamic Systems . . . . . . . . . . . . . . 35

2.2 .2 Chaotic Systems . . . . . . . . . . . . . . 36

2.2 .3 Attractors . . . . . . . . . . . . . . 37

2.2 .4 Lyapunov Exponents . . . . . . . . . . . . . . . . 39

2.2 .5 Bifurcation . . . . . . . . . . . . . . 40

2.2 .6 Ergodic and Mixing Systems . . . . . . . . . . . . . . . 40

2.2 .7 Binary Chaos . . . . . . . . . . . . . . 41

2.3 Relationship between Chaos and Cryptography . . . . . . . . . 43

3 Randomness, Complexity and Chaos. $\quad 45$

3.1 Informal Overview . . . . . . . . . . . . . . . . 45

3.2 Complexity Theory Approach . . . . . . . . . . . . . . . 48

3.2 .1 Turing Machine . . . . . . . . . . . . . . . . . . . 48

3.2 .2 Algorithmic Complexity . . . . . . . . . . . . . . 50

3.2.3 Compressibility and Algorithmic Randomness . . . . . . . . 50

3.2 .4 Symbolic Complexity . . . . . . . . . . . . . . . . 51

3.3 Information Theory Approach . . . . . . . . . . . . . 51

3.3 .1 True Randomness . . . . . . . . . . . . . . . . . . . . 52

3.3 .2 Shannon Entropy . . . . . . . . . . . . . . . 52

3.3.3 Entropy-Complexity Relationship . . . . . . . . . . . . . 53

3.4 Entropy and Complexity of Chaotic Systems . . . . . . . . . . . . . 54

3.4.1 Partitioning and Symbolic Dynamics . . . . . . . . . . . . 54

3.4 .2 Kolmogorov-Sinai Entropy . . . . . . . . . . . . . . 54

3.4.3 Complexity of the Trajectory . . . . . . . . . . . . 55

3.5 Pseudo-Randomness . . . . . . . . . . . . . . . . 56

3.5.1 Probabilistic Ensembles . . . . . . . . . . . . . 56

3.5 .2 One-Way Function . . . . . . . . . . . . . . 57

3.5.3 Pseudo-Random Generators . . . . . . . . . . . . . . . . 59

3.6 Chaos-based Pseudo-Random Generators . . . . . . . . . . . . . . . 61 
4 Pseudo-Chaotic Cryptosystems $\quad 65$

4.1 Chaos and Pseudo-Chaos . . . . . . . . . . . . . . 65

4.2 Floating-point Approximations of Chaos . . . . . . . . . . . 68

4.2.1 Partitioning the State Space . . . . . . . . . . . . . 71

4.2 .2 Chebyshev Map . . . . . . . . . . . . . . . . . . 71

4.2 .3 Logistic Map . . . . . . . . . . . . . . . . . . 72

4.2 .4 Tent Map . . . . . . . . . . . . . . . . 76

4.2 .5 Other Chaotic Maps . . . . . . . . . . . . . . . . 78

4.2 .6 Multi-Stream Generators . . . . . . . . . . . . . . . . 79

4.2 .7 Iteration Counting Ciphers . . . . . . . . . . . . . . . 81

4.2.8 Multi-valued Chaotic Maps

with Analytical Solutions . . . . . . . . . . . . . 82

4.3 Binary Pseudo-Chaos . . . . . . . . . . . . . . . . . . 84

4.3 .1 RANROT . . . . . . . . . . . . . . . 85

4.3 .2 Discrete Tent Map . . . . . . . . . . . . . . . . . 86

4.3 .3 Cellular Automata . . . . . . . . . . . . . . . 86

4.3 .4 Generalized Baker Map . . . . . . . . . . . . . . . . 87

4.3.5 Pseudo-Chaos and Conventional Cryptosystems . . . . . . 89

5 E-Larm: A Chaos Based Encryption System 91

5.1 Overview . . . . . . . . . . . . . . . . 91

5.2 Algorithm . . . . . . . . . . . . . . . . . . 91

5.3 Practical Implementation . . . . . . . . . . . . . . 95

5.3 .1 Platform . . . . . . . . . . . . . . . 95

5.3 .2 User Interface . . . . . . . . . . . . . . . . . . . 95

5.3 .3 Sessions . . . . . . . . . . . . . . . . 97

5.4 Initial Conditions and Parameters . . . . . . . . . . . . . 100

5.5 Evaluation . . . . . . . . . . . . . . . . . 101

6 Evaluation of Chaos-based PRNG's 102

6.1 Introduction . . . . . . . . . . . . . . . 102

6.2 Considerations for

Randomness and Unpredictability . . . . . . . . . . . . . . . . 106 
6.3 Chaotic Maps . . . . . . . . . . . . . . . . . . 106

6.4 Visual Approaches . . . . . . . . . . . . . . . . . 107

6.4 .1 Phase-Space Diagrams . . . . . . . . . . . . . 107

6.4 .2 Time series . . . . . . . . . . . . . . . 108

6.4 .3 Power Spectrum . . . . . . . . . . . . . . . . 108

6.4.4 Approximated Entropy . . . . . . . . . . . . . . . . 109

6.5 Brief Description of Tests . . . . . . . . . . . . . 111

6.6 Performance Evaluation . . . . . . . . . . . . . . . 130

6.7 Conclusions . . . . . . . . . . . . . . . . 130

7 Conclusions 132

7.1 Theory of Chaos based Cryptography . . . . . . . . . . . . . 132

7.2 Practical Issues . . . . . . . . . . . . . . . . . . . . 134

7.3 Future Work . . . . . . . . . . . . . . . . . 136

$\begin{array}{lr}\text { Bibliography } & 138\end{array}$

$\begin{array}{lr}\text { Index } & 149\end{array}$ 


\section{List of Figures}

1.1 The cross-disciplinary approach to cryptography . . . . . . . . . 18

1.2 Self-organization of the nature . . . . . . . . . . . . 20

1.3 Production of pastry dough $[23] \ldots \ldots 21$

2.1 An iterative cryptosystem . . . . . . . . . . . . 25

2.2 A secure communication scheme using encryption and decryption. . . 26

2.3 A trajectory of a block cipher. The block cipher iterates exactly $N$ times. The plaintext $p$ is assigned to the initial state. The ciphertext $c$ is the final state. The key is determined by parameters of the iterated function (not shown on the diagram). . . . . . . . . . . . . 30

2.4 Trajectories of stream ciphers based on a single trajectory. (a) The ciphertext $c$ is the plaintext $p$ plus chaotic noise $x$ (e.g. simple XOR cipher using chaos key stream suggested by Bianco, see Section 4.2.3); (b) The ciphertext $c$ is a number of iterations $n$ (Baptista, Section 4.2.7); (c) The ciphertext $c$ is the system state after $p$ iterations (Gallagher, Section 4.2.7) . . . . . . . . . . . . . . 30

2.5 A two-dimensional chaotic system: (a) Time space; (b) Phase space. 37

2.6 Binary Chaos: the sensitivity to the initial condition (a) and the topological transitivity (b) are defined on the space $\Omega$. . . . . . . . . 42

2.7 Phase portraits of chaotic and cryptographic systems.A chaotic system (left) usually has an attractor with a fractal dimension (less than the number of independent variables). A cryptographic system (right) attempts to maximize the dimension up to an integer. . . . . . . . . 43

3.1 Relationship between the concepts. . . . . . . . . . . . . . . 46 
3.2 Scale of the entropy . . . . . . . . . . . . . . . . . 47

3.3 A synergy between a chaotic system (top: $\approx$ is a rounding function, $\hat{x_{n}}$ is the output) and a PRNG (bottom: $b$ is a hard-core predicate, $y_{n}$ is the output). ................... 60

3.4 A rarefied sample for $k=6 \ldots \ldots \ldots 2$

3.5 The sawteeth map for $p=1279$ and $q=255 \ldots \ldots 63$

4.1 Properties of chaotic and pseudo-chaotic systems. . . . . . . . . 66

4.2 Examples of orbits of a pseudo-chaotic system. (a) Dangerously short orbits (unsuitable for cryptography); (b) A single orbit (the best choice for cryptography); (c) Multiple orbits with the same length (also suitable for encryption). . . . . . . . . . . . . . . 67

4.3 A Laypunov exponent of a chaotic (a) and pseudo-chaotic (b) system 68

4.4 Trajectories of a continuous-state chaotic system (4.4) and its 64-bit floating-point approximation. The first curve is obtained by means of the analytical solution (4.5). The rounding off error is amplified in each iteration and the trajectories diverge exponentially. . . . . . . 69

4.5 The average and the minimal cycle length of the logistic system (4.4) verses floating-point precision obtained from 10 samples of the logistic system. . . . . . . . . . . . . . . . . . . 70

4.6 The empirical Cumulative Distribution Function of a sequence generated with the Chebyshev system before (doted line) and after (solid line) the additional transformation (4.3). The straight line looks like the uniform distribution on $(-2,2) \ldots \ldots \ldots 72$

4.7 The logistic map for $r=0.99 \ldots \ldots \ldots 73$

4.8 A chaotic sequence generated with the logistic map for $x_{0}=0.34$ and

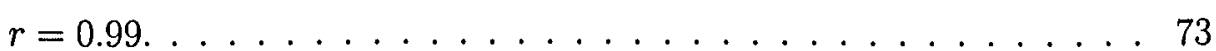

4.9 Bifurcation of the logistic map. The most 'unpredictable' behavior occurs when $r \rightarrow 1 \ldots \ldots \ldots \ldots \ldots$

4.10 Attractor points corresponding to different values of the parameter $r$ in the Matthews map. . . . . . . . . . . . . . . . 74

4.11 The analytical solution at $n=5$ of the logistic map for $r=1 . \ldots 75$ 
4.12 The Probability Density Function of a state sequence produced by the logistic system with an incomplete partition. . . . . . . . 76

4.13 The tent map. . . . . . . . . . . . . . . . . 77

4.14 The Gallagher map. . . . . . . . . . . . . . . . . 78

4.15 A multi-stream cryptographic generator . . . . . . . . . . . . 80

4.16 A multiple valued chaotic map $x_{n+1}=f\left(x_{n}\right) \ldots \ldots 83$

4.17 A pseudo-random generator based on an analytic solution. . . . . . . 84

4.18 The discrete tent map. . . . . . . . . . . . . . . . . 86

4.19 A stretch-and-fold baker transformation. . . . . . . . . . . . . . . 87

4.20 Six Baker transformations at $\rho=\{0.25,0.5,0.25\}$ applied to Lenna [89]. . . . . . . . . . . . . . . . . . . . 88

4.21 A typical block cipher is a combination of several pseudo-chaotic systems . . . . . . . . . . . . . . . . . . . . 89

5.1 E-Larm GUI . . . . . . . . . . . . . . . . . . . . . 97

6.1 Properties of the logistic map . . . . . . . . . . . . . 110

6.2 Properties of the logistic map (cont.) . . . . . . . . . . . . 115

6.3 Properties of the sine map . . . . . . . . . . . . 116

6.4 Properties of the tangent-feedback map . . . . . . . . . . . 117

6.5 Properties of the logarithm-feedback map . . . . . . . . . . . . 118

6.6 Properties of the tent map . . . . . . . . . . . . . . . . . 119

6.7 Properties of the sawteeth map . . . . . . . . . . . 120

6.8 Properties of the sawteeth $\operatorname{map}$ (cont.) . . . . . . . . . . . . 121

6.9 Properties of the exactly solvable map . . . . . . . . . . . . . . 122

6.10 Properties of the exactly solvable map (cont.) . . . . . . . . . . . . . 123

6.11 Approximated entropy plots . . . . . . . . . . . . . . . . 124

6.12 Estimation of $P$-value for sha-1, par, par-bad, sin . . . . . . . . 125

6.13 Estimation of $P$-value for sha-1, par, par-bad, sin (cont.) . . . . . 126

6.14 Estimation of $P$-value for $\tan , \log$, saw, mix $\ldots \ldots \ldots 127$

6.15 Estimation of $P$-value for tan, log,saw,mix (cont.) . . . . . . . . . 128

6.16 Estimation of $P$-value for $\operatorname{mix}$ (long sequence) . . . . . . . . . . 129

6.17 Performance evaluation of pseudo-random generators . . . . . . . 130 


\section{Chapter 1}

\section{Introduction}

\subsection{The Information Society}

Our digital age has brought about a number of changes in society but perhaps the most profound is the impact it has had upon basic human activities such as decision making, information processing and communications which are all supported by computer devices.

The information revolution can be compared in its impact to that of the industrial revolution of the nineteenth century. The value of information has changed in that both the constructive and destructive powers of information flow are very different compared with the situation less than a hundred years ago. In the commercial sector, 'know how' contributes considerably to company market value because information provides a primary competitive advantage. Clearly, critical information is vital for national security and financial organizations. Accurate knowledge of public opinion allows political leaders to react rapidly in their policies or programs. Thus, political power is now critically dependent on the flow of information.

Organisations in both the public and private sectors have become increasingly dependent on electronic data processing. Vast amounts of digital data are now gathered and stored in large computer data bases and transmitted between computers and terminal devices linked together in complex communications networks. Without appropriate safeguards, these data are susceptible to interception during transmission, or they may be physically removed or copied while in storage. This could result in 
unwanted exposures of data and potential invasions of privacy. Data are also susceptible to unauthorised deletion, modification, or addition during transmission or storage. This can result in illicit access to computing resources and services, falsification of personal data or business records, or the conduct of fraudulent transactions, including increases in credit authorisations, modification of funds transfers, and the issue of unauthorised payments.

The speed of information flow has developed considerably over the past ten years. As with high speed vehicles, skilled control becomes increasingly important as information flow increases in the rapidity of its exchange; the web being an environment in which information flow is now very difficult to control effectively.

Access to the global market is a key incentive in society. New economic incentives require new services based on electronic and mobile transactions and both individuals and industries will require involvement under conditions that are not only financially advantageous but secure. The continuous update of security infrastructures is absolutely necessary to encourage the further development of the new global economy.

Modern information security manifests itself in many ways according to the situation and requirement. It deals with such concepts as confidentiality, data integrity, access control, identification, authentication and authorization. Practical applications, closely related to information security, are private messaging, financial transactions, online services and many others.

Legislators, recognizing that the importance of information security, have passed laws to help prevent these problems. But laws alone cannot prevent attacks or eliminate threats to data processing systems. Additional steps must be taken to preserve the secrecy and integrity of computer data. Among the security measures that should be considered is cryptography, which embraces methods for rendering data unintelligible to unauthorised parties.

\subsection{Cryptography, Cryptanalysis and Cryptology}

The word is derived from the Greek kryptos, meaning 'hidden', and graphein meaning 'to write'. Cryptography concerns the ways in which communications and data can 
be encoded to prevent disclosure of their contents through eavesdropping or message interception, using codes, cyphers, and other methods.

From ancient civilisations, with Julius Caesar, Abraham Lincoln and Iosif Stalin cryptography has been a part of history. During the World War II, the Germans developed the Enigma machine to have secure communications. Enigma codes were decrypted first in Poland in the late 1930s and then under the secret 'Ultra Project' based at Bletchly Park in Buckinghamshire (UK) during the early 1940s. This led to a substantial reduction in the level of allied shipping sunk by German U-boats and together the invention of Radar was arguably one of the most important contributions that electronics made to the war effort. In addition, this work contributed significantly to the development of electronic computing after 1945.

Cryptography has always been shrouded in secrecy itself and remains one of the most secret sciences in the world. Professional cryptographers working for intelligence services and commercial organizations have been limited in their publications. As a result, the freely available literature never fully reflects the state of the art. Nations vary in their reticence: whereas the United States released quite generous information on the situation in the Second World War, the Soviet Union clocked itself in silence; but all the time scientists from both countries kept abreast of each other. Claude Shannon, an American electrical engineer, formulated the major criteria and fundamental of cryptographic techniques in his secret report of 1945 that was unclassified in 1949 and published as Communication theory of secrecy systems [92]. Vladimir Alexandrovich Kotelnikov, a founder of Russian cryptography, in 1941 formulated the requirements to a perfect encryption system and mathematically proved its cryptographic resistance. This work together with the Kotelnikov theorem (the sampling theorem) became the foundation of Russian cryptography and provided secure communication during World War II in 1942-1945.

Since that time, cryptography has absorbed ideas from complexity theory, number theory, group theory, combinatory logic and modern computer science and grown from basic symmetric ciphers to Public Key Infrastructure (PKI) and complex cryptosystems. In particular, in 1976 asymmetric public keys was first proposed in a revolutionary article by Whitfield Diffie and Martin Hellman [39].

A century ago, one could say that cryptography is the science (or art) of secret 
writing and reading, which has grown from semiotics, the study of signs and signusing behavior ${ }^{1}$, rather than mathematics.

Today cryptography is, essentially, the study of mathematical and computational techniques underlying information security. Cryptography is closely connected to the disciplines of cryptanalysis and cryptology. In simple terms, cryptanalysis is the art of breaking cryptosystems, i.e. retrieving the original message without knowing the proper key or forging an electronic signature. Cryptology is the mathematics, such a number theory, which underpin cryptography and cryptanalysis.

Cryptography is the only known practical method for protecting information transmitted through communications networks that uses land lines, communications satellites, and microwave facilities. In some instances, it can be the most economical way to protect stored data. Cryptographic procedures can also be used for message authentication, digital signatures and personal identification for authorising electronic funds transfer and credit card transactions.

\subsection{The Synergetic Approach to Cryptography}

In making innovations, we typically take existing models from one science and transplant them into a new subject area. For example, it is quite natural to apply models from nonlinear dynamics (chaos theory) for the purpose of encryption. If we are successful, a new cryptographic algorithm will emerge. This is a practical benefit of a cross-disciplinary approach (Figure 1.1).

Unlike most new disciplines that appear at the edge, of existing sciences when a model or a technique from one subject area is applied in another, synergetics studies common fundamentals of these sciences, extending the global collection of ideas and methods [2].

The term synergetics (from Greek: synergeia - working together, cooperation) was introduced by the German physicist Haken in the beginning of 70's. Haken's

\footnotetext{
${ }^{1}$ Although the word was used in this sense in the 17th century by the English philosopher John Locke, the idea of semiotics as an interdisciplinary mode for examining phenomena in different fields emerged only in the late 19 th and early 20 th centuries.
} 


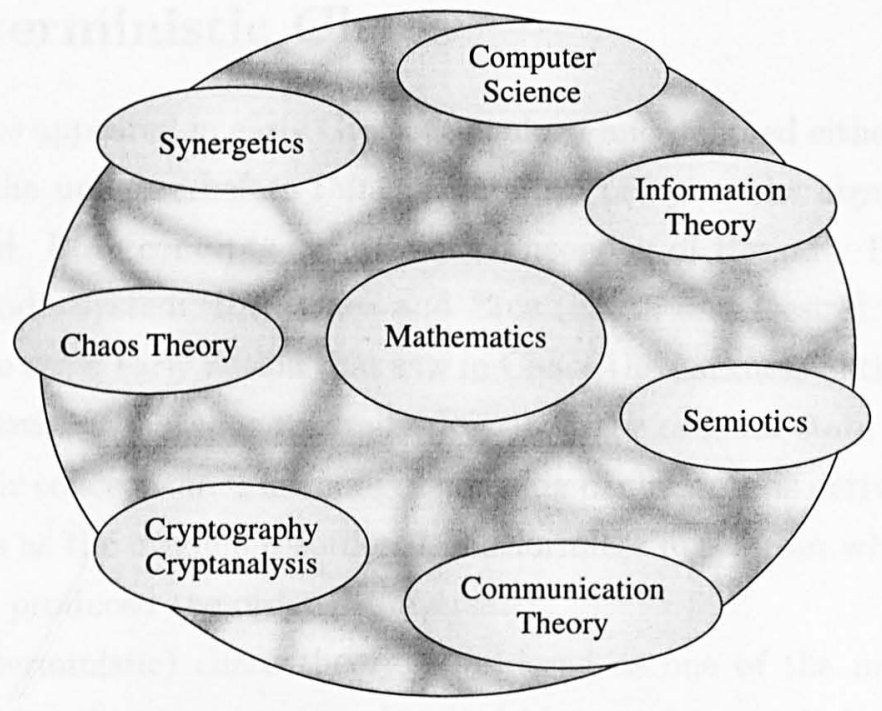

Figure 1.1: The cross-disciplinary approach to cryptography

synergetics treats systems in which cooperation among subsystems creates organized structure on macroscopic scales [54]. Synergetics aims to understand common laws driving processes in different nonlinear systems with a feedback. Examples of problems studied by synergetics are bifurcations, phase transitions in physics, nonlinear oscillations in electrical circuits, population dynamics. From a certain view point, a similiar definition is given by the American scientist R. Buckminster Fuller: "Synergy means behavior of whole systems unpredicted by the behavior of their parts taken separately" [47]. One can see this property in cryptographic systems, whose strength depends on the integrity of several mathematical constructions.

Today, it would not be easy to find a discipline remaining untouched by synergetics and deterministic chaos. These new sciences have transfused all the scope of human knowledge: not only mathematics, physics, biology, economics, digital imaging, simulation sciences $[20,6,18,4,16]$, but also many human studies such as history and sociology $[1,20]$. Cryptography, of course, was not an exception - a number of scientists have relatively evaluated encryption techniques based on nonlinear dynamic systems (for example, [76, 53, 69, 45, 63]). 


\subsection{Deterministic Chaos}

The word chaos appeared in early Greek cosmology and denoted either the primeval emptiness of the universe before things came into being or the abyss of Tartarus, the underworld. Both concepts occur in the Theogony of Hesiod ${ }^{2}$. First there was Chaos in Hesiod's system, then Gaea and Eros (Earth and Desire). This concept tied in with the other early notion that saw in Chaos the darkness of the underworld.

In later cosmologies, chaos generally described the original state of things irrespective of their conception. The modern meaning of the word is derived from Ovid, who saw chaos as the original disordered and formless mass from which the maker of the Cosmos produced the ordered universe.

Today (deterministic) chaos theory, considered as one of the most important sciences of the last few decades, has absorbed ideas and methods from pure mathematics, dynamical systems theory, symbolic dynamics, non-equilibrium thermodynamics and fractal geometry. Compared with Greek cosmology, it denotes the opposite properties of chaos: self-organization, order, bounded space and determinism. However, both meanings of the word assume the unpredictability.

The idea that many simple nonlinear deterministic systems can behave in an apparently unpredictable and chaotic manner was first observed by the great French mathematician Henri Poincaré. Other early pioneering works in the field of chaotic dynamics are to be found in the mathematical literature by such luminaries as Birkhoff, Cartwright, Littlewood, Levinson, Smale, Kolmogorov and his students.

The key feature of chaotic behavior in different systems is mainly related to the high sensitivity to initial conditions due to exponential divergence of all trajectories lying on the attracting structure which is normally bounded in an appropriate phase space. In the 1960's Edward Lorenz, an American meteorologist, discovered a stable chaotic attractor and predicted that: “. . it may happen that small differences in the initial conditions produce very great ones in the final phenomena. A small error in the former will produce an enormous error in the future. Prediction becomes impossible..."

The Nobel laureate Ilia Prigogine, a Russian-born Belgian physical chemist, is

\footnotetext{
${ }^{2}$ Hesiod, $700 \mathrm{BC}$, one of the earliest Greek poets. His epic Theogony describes the myths of the gods.
} 


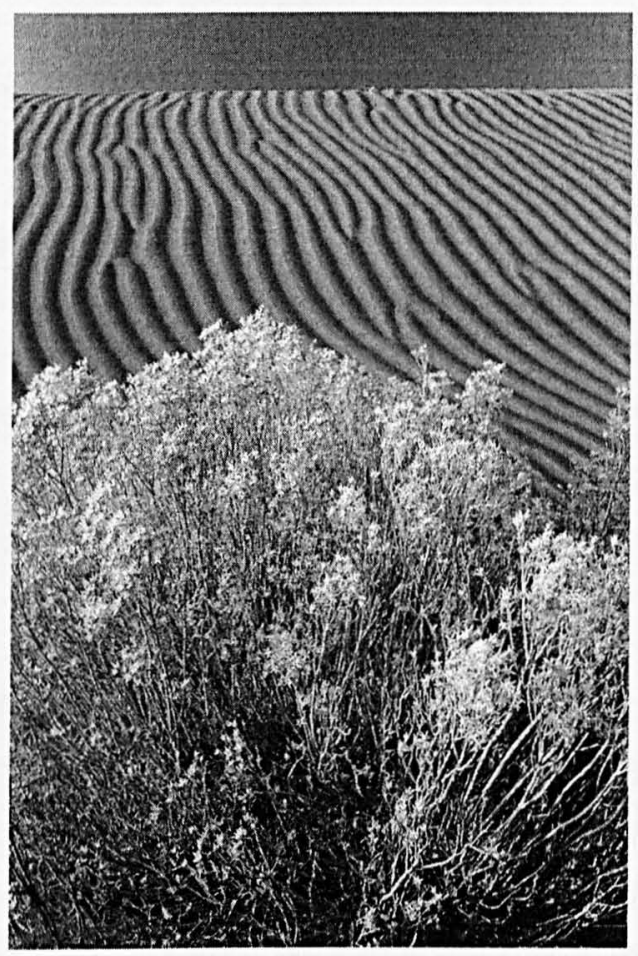

Figure 1.2: Self-organization of the nature 


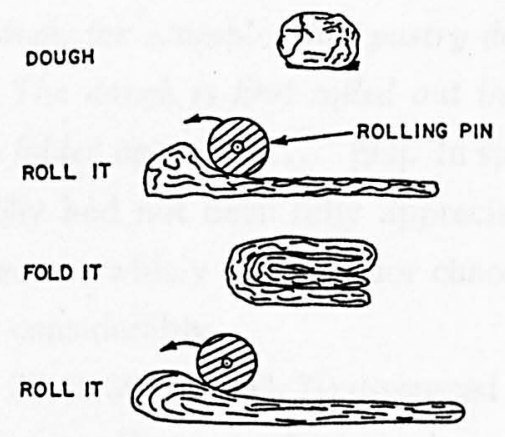

Figure 1.3: Production of pastry dough [23]

one of the founders of chaos theory. His research in nonlinear dissipative structures led to the concept of equilibrium and non-equilibrium conditions to categorize the state of a system. In the physical studies of thermodynamics, Prigogines' research revealed non-equilibrium conditions that led to systemic behavior different from what was expected by the customary interpretation of the second law of thermodynamics. The phenomena of bifurcation and self-organization emerged from systems in equilibrium as if there was disruption or interference. This disruption or interference became the next step in chaos theory; it became chaos/complexity theory.

Mathematical or deterministic chaos is a dynamic system, characterized with a 'complex' and 'unpredictable' behavior. Intuitively, this property suits the requirements of a digital encryption system - on the one hand computer-based cryptosystems are deterministic; on the other, they must be cryptographically unpredictable. Practically, the last property implies that given certain information on the ciphertext and the plaintext (the message), a cryptanalyst should not be able to predict the cryptographic transformation and recover the key or the message.

\subsection{History of Chaos-based Cryptography}

In his classical paper Claude Shannon explicitly mentioned the basic stretch-andfold mechanism of chaos for the purpose of encryption (Figure 1.3): "Good mixing transformations are often formed by repeated products of two simple noncommuting 
operations. Hopf ${ }^{3}$ has shown, for example, that pastry dough can be mixed by such a sequence of operations. The dough is first rolled out into a thin slab, then folded over, then rolled, and then folded again, etc..." [92]. In spite of this, the importance of chaos-based cryptography had not been fully appreciated till the end of 1980's when digital computers became widely available for chaos simulations and the role of cryptography increased considerably.

Many papers (e.g. [67, 33, 3, 36, 104, 93, 7]) discussed encryption schemes based on synchronized chaotic circuits. However, these analog schemes belong more to the field of steganography and secure radio communication, and lie beyond the scope of this thesis.

Cryptography attracted scientists from many areas and they started exploiting different dynamic systems for the purpose of encryption. Most of these systems were defined on real numbers, so floating-point arithmetic was used to approximate chaos on a finite-state machine (digital computer) [41, 59, 76, 25, 53, 82, 49, 22, 103, 69].

Discrete (binary) chaotic systems [97] such as the cellular automata [102, 52], discrete Baker transformation [89, 45], discrete tent map [75] and discrete affine transformation [84] provided more efficient encryption schemes.

Since 2000, the potential of chaos-based communication, especially spread spectrum modulation, has been recognized worldwide. For instance, the book Chaotic electronics in telecommunications by Kennedy et al. [63] described chaotic modulations and suggested their electronic implementations. Again, the emphasis here is put on information coding rather then digital encryption, which is the subject of this research.

Finally, inspiration for this work came from my supervisor J. M. Blackledge [26], L. Kocarev [66], Z. Kotulski and J. Szczepański [68, 95].

\footnotetext{
${ }^{3}$ Hopf, Eberhard F. F, (1902-1983), an Austrian mathematician who made significant contributions in topology and ergodic theory, studied 'pastry dough mixing' in compact spaces [On causality, statistics and probability, Journal of Mathematics and Physics, 13, pp. 51-102, 1934]
} 


\subsection{Original Contribution}

This thesis discusses the theoretical background and practical implementations of chaos-based cryptosystems known at the present moment. Additionally, the following results are considered useful and unique:

- Floating-point approximations of various chaotic systems (i.e. pseudo-chaos) are studied in deep using different types of partitioning methods and statistical tests.

- Known cryptographic techniques using chaos have been combined and extended into an original system E-Larm. In particular,

i multiple nonlinear chaotic systems are used together to increase the reliability and complexity of the generator;

ii a mixing component is used to provide a relationship between different chaotic systems and increase the average cycle length of the generator in general;

iii the minimal bound of the iteration counter (discrete time) is used to improve the pseudo-random appearance of the output; whereas the upper bound is used to avoid very short orbits;

iv optimal values for algorithm parameters are derived and implemented.

- An evaluation of the E-Larm system is given using a test methodology based on NIST (National Institute of Standards and Technologies) recommendations.

- Conclusions are made about the general role of chaos theory in cryptography. 


\section{Chapter 2}

\section{Preliminaries}

This chapter assembles the mathematical concepts from cryptography [90, 77] and nonlinear dynamics $[81,11,12,9,4,31,79]$, emphasizing their common fundamentals.

\subsection{Cryptography}

\subsubsection{What is Digital Information?}

With regard to information in a computer environment we assume an array of bits or binary strings that carry a text message or any multimedia object such as an image and sound. A binary string to be transmitted or kept confidential in a storage is called plaintext. We denote the plaintext as

$$
p=\left\{p_{1}, p_{2}, \ldots, p_{i}, \ldots, p_{n} \mid p_{i} \in \mathcal{P}\right\}
$$

where $\mathcal{P}$ is a finite alphabet of plaintext symbols. In digital cryptosystems, the alphabet is binary, that is $\mathcal{P}=\mathbb{Z}_{2}=\{0,1\}$. We can also consider the alphabet of bytes $\left(\mathcal{P}=\mathbb{Z}_{256}=\{0,1\}^{8}\right)$ or larger data blocks.

Let $c=\left\{c_{1}, c_{2}, \ldots, c_{i}, \ldots, c_{n} \mid c_{i} \in \mathcal{C}\right\}$ denote the encrypted message or the ciphertext. The alphabet $\mathcal{C}$ can differ from $\mathcal{P}$, in most cases, $\mathcal{P}=\mathcal{C}=\{0,1\}^{m}$. 


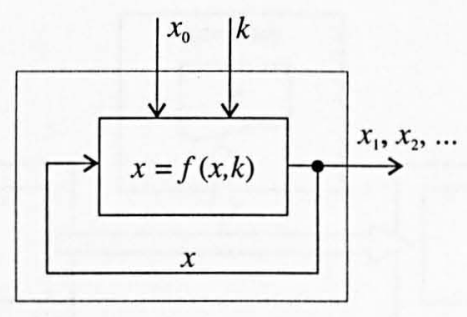

Figure 2.1: An iterative cryptosystem

\subsubsection{Cryptographic System}

In a broad sense, a cryptographic system is the whole computer infrastructure providing information security, i.e. a balanced software and hardware solution implementing cryptographic algorithms (encryption/decryption, authentication, key management etc). In the real world a cryptographic system crucially depends on humans.

In a narrow sense, a cryptographic system is a mathematical construction that transforms information to make it inaccessible for an adversary. A cryptosystem can be considered as a dynamic system (Section 2.2.1)

$$
\mathcal{S}=\langle X, f: X \times \mathcal{K} \rightarrow X\rangle
$$

where the set $X$ is a state space and the function $f$ is a cryptographic transformation depending on a key from $\mathcal{K}$. The system state $x \in X$ encodes certain information, for example, a plaintext or a signature (in most ciphers, $X=\mathcal{P}=\mathcal{C}$ ). Other examples of cryptographic systems are a pseudo-random generator and hash functions. The function $f$ is given by a deterministic algorithm, which can be implemented on a Turing machine.

Often, a cryptosystem is defined by a nonlinear iterative transformation with a feedback (Figure 2.1). The system produces a sequence of states $x_{0}, x_{1}, \ldots, x_{n}, \ldots$, where $x_{n}=f\left(x_{n-1}, k\right)=f^{n}\left(x_{0}, k\right), x_{0} \in X, k \in \mathcal{K}$. The initial condition $x_{0}$ is an input message, and the final state $x_{n}$ is an output message. The sequence $\left\{x_{n}\right\}$ is called a trajectory or an orbit. Since $f$ is deterministic, the whole trajectory is given by the initial condition $x_{0}$ and the (secret) parameter $k$. 


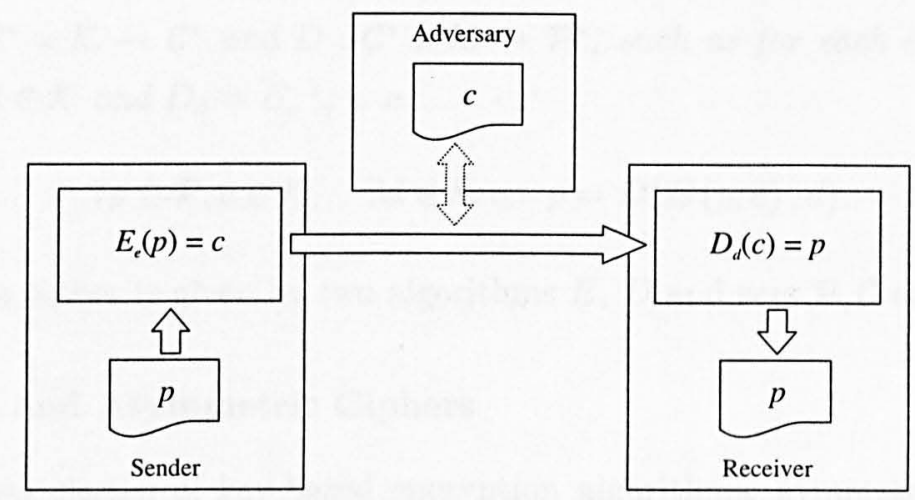

Figure 2.2: A secure communication scheme using encryption and decryption.

So far, any conventional cryptographic system can be considered as a deterministic dynamic system $\langle f, X, \mathcal{K}\rangle$ with a nonlinear iterated function $f$, a state space $X$ and a key space $\mathcal{K}$. In general, the basic requirements for a cryptosystem are unpredictability and random-like behavior.

\subsubsection{Encryption and Decryption}

Encryption is a transformation $E: \mathcal{P}^{*} \times \mathcal{E} \rightarrow \mathcal{C}^{*}$, where $\mathcal{C}$ is a ciphertext alphabet, and $\mathcal{E}$ is an encryption key space. Hence,

$$
c=E(p, e)=E_{e}(p), \quad p \in \mathcal{P}, c \in \mathcal{C}, e \in \mathcal{E} .
$$

Decryption is the inverse transformation $D: \mathcal{C}^{*} \times \mathcal{D} \rightarrow \mathcal{P}^{*}$, where $\mathcal{D}$ is a decryption key space, i. e.

$$
p=D(c, d)=D_{d}(c), \quad p \in \mathcal{P}, c \in \mathcal{C}, d \in \mathcal{D} .
$$

Usually, $\mathcal{E}=\mathcal{D}=\mathcal{K}=\{0,1\}^{m}$.

Figure 2.2 gives a classical communication scheme using encryption and decryption.

Definition 1 Encryption scheme or cipher is a system

$$
\mathcal{S}=\langle E, D, \mathcal{P}, \mathcal{C}, \mathcal{K}\rangle
$$


where $E: \mathcal{P}^{*} \times \mathcal{K} \rightarrow \mathcal{C}^{*}$ and $D: \mathcal{C}^{*} \times \mathcal{K} \rightarrow \mathcal{P}^{*}$, such as for each $e \in \mathcal{K}$ exists a unique key $d \in \mathcal{K}$ and $D_{d}=E_{e}^{-1}$, i. e.

$$
\forall p \in \mathcal{P}, e \in \mathcal{K}, \quad \exists d \in \mathcal{K}: \quad p=D(E(p, e), d)
$$

Practically, a cipher is given by two algorithms $E, D$ and sets $\mathcal{P}, \mathcal{C}$ and $\mathcal{K}$.

\section{Symmetric and Asymmetric Ciphers}

There are two classes of key-based encryption algorithms, symmetric (or private key) and asymmetric (or public key) algorithms. The difference is that symmetric algorithms use the same key for encryption and decryption ( $e=d$, or the decryption key is easily derived from the encryption key), whereas asymmetric algorithms use a different key for encryption and decryption $(e \neq d)$, and the decryption key cannot be derived from the encryption key.

Asymmetric ciphers (also called public-key algorithms or generally public-key cryptography) permit the encryption key to be public (it can even be published in a newspaper), allowing anyone to encrypt with the key, whereas only the proper recipient (who knows the decryption key) can decrypt the message. The encryption key is also called the public key and the decryption key the private or secret key. A well known example of asymmetric cipher is cryptographic system developed in 1977 by Rivest, Shamir and Adleman (RSA) in the US [87]. Pretty Good Privacy (PGP), an encryption software written by Philip R. Zimmerman, is an other example of public key cryptography [106].

This paper focuses on symmetric ciphers.

\section{Substitution and Transposition Ciphers}

Before the development of digital computers, cryptography consisted of characterbased algorithms. Different cryptographic algorithms either substituted characters for one another or transposed characters with one another. The better algorithms did both, many times each.

Although the technology for developing cypher systems is more complex now, the underlying philosophy remains the same. The primary change is that algorithms 
work on bits instead of characters. This is actually just a change in the alphabet size-from 26 elements to $2^{256}$ elements in modern block ciphers.

Substitution Cyphers As their name suggests, these preserve the order of the plaintext symbols, but disguise them. Each letter or group of letters is replaced by another letter or group to disguise it. In its simplest form, 'a' becomes ' $D$ ', 'b' becomes ' $E$ ', 'c' becomes ' $F$ ' etc.

More complex substitutions can be devised, e.g. a random (or key controlled) mapping of one letter to another. This general system is called a monoalphabetic substitution. They are relatively easy to decode if the statistical properties of natural languages are used. For example, in English, 'e' is the most common letter followed by ' $t$ ', then ' $a$ ' etc.

The cryptanalyst would count the relative occurrences of the letter in the cyphertext, or look for a word that would be expected in the message. To make the encryption more secure, a polyalphabetic cypher may be used, in which a matrix of alphabets is employed to smooth out the frequencies of the cyphertext letters.

It is in fact possible to construct an unbreakable cypher if the key is longer than the plaintext, although this method, known as a 'one time pad' has practical disadvantages.

Transposition Cyphers A common example, the 'column transposition cypher' is shown in Table 2.1 Here the plaintext is: 'This is an example of a simple transposition cypher'. The cyphertext is: 'almniefheolpnatnepsorimsripdspiathesaatsicixfeocb'

The plaintext is ordered in rows under the key which numbers the columns so formed. Column 1 in the example is under the key letter closest to the start of the alphabet. The cyphertext is then read out by columns, starting with the column whose number is the lowest.

To break such a cypher, the cryptanalyst must guess the length of the keyword, and order of the columns. 


$\begin{array}{lllllll}\mathrm{K} & \mathrm{E} & \mathrm{Y} & \mathrm{W} & \mathrm{O} & \mathrm{R} & \mathrm{D} \\ 3 & 2 & 7 & 6 & 4 & 5 & 1 \\ \mathrm{t} & \mathrm{h} & \mathrm{i} & \mathrm{s} & \mathrm{i} & \mathrm{s} & \mathrm{a} \\ \mathrm{n} & \mathrm{e} & \mathrm{x} & \mathrm{a} & \mathrm{m} & \mathrm{p} & \mathrm{l} \\ \mathrm{e} & \mathrm{o} & \mathrm{f} & \mathrm{a} & \mathrm{s} & \mathrm{i} & \mathrm{m} \\ \mathrm{p} & \mathrm{l} & \mathrm{e} & \mathrm{t} & \mathrm{r} & \mathrm{a} & \mathrm{n} \\ \mathrm{s} & \mathrm{p} & \mathrm{o} & \mathrm{s} & \mathrm{i} & \mathrm{t} & \mathrm{i} \\ \mathrm{o} & \mathrm{n} & \mathrm{c} & \mathrm{i} & \mathrm{p} & \mathrm{h} & \mathrm{e} \\ \mathrm{r} & \mathrm{a} & \mathrm{b} & \mathrm{c} & \mathrm{d} & \mathrm{e} & \mathrm{f}\end{array}$

Table 2.1: Example of Transposition Cypher

\section{Block and Stream Ciphers}

Encryption algorithms can also be divided into stream ciphers and block ciphers. Stream ciphers (also known as state ciphers) encrypt a single bit or block of plaintext at a time and the encryption depends on time (i.e. cipher state); identical plaintext blocks are transformed into different ciphertext blocks. Block ciphers take a larger block of bits and encrypt them as independent units; identical plaintext blocks correspond to identical ciphertext blocks. For example, the transposition cipher is a typical block encryption scheme.

A stream or block cipher can be considered as a nonlinear dynamic system (2.1). The role of trajectory in each class of ciphers is explained below:

1. A block cipher performs $n$ iterations with an invertible function $f$ (Figure 2.3). The number $n$ is fixed and relatively small (often, $n=16$ ). Each iteration transfers the system into the next state, i. e. $x_{i+1}=f\left(x_{i}\right)$. The plaintext is assign to the initial condition $\left(x_{0}=p\right)$, and the ciphertext is obtained from the final state $\left(c=x_{n}\right)$. The intermediate states $\left(x_{1}, \ldots, x_{n-1}\right)$ are hidden from external observers. Symbolically,

$$
\begin{gathered}
E(p, k)=f^{n}(p, k), \\
D(c, k)=f^{-n}(c, k) .
\end{gathered}
$$

Modern block ciphers combine several dynamic systems by adding their states 


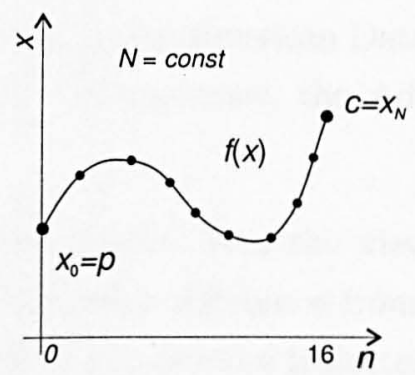

Figure 2.3: A trajectory of a block cipher. The block cipher iterates exactly $N$ times. The plaintext $p$ is assigned to the initial state. The ciphertext $c$ is the final state. The key is determined by parameters of the iterated function (not shown on the diagram).
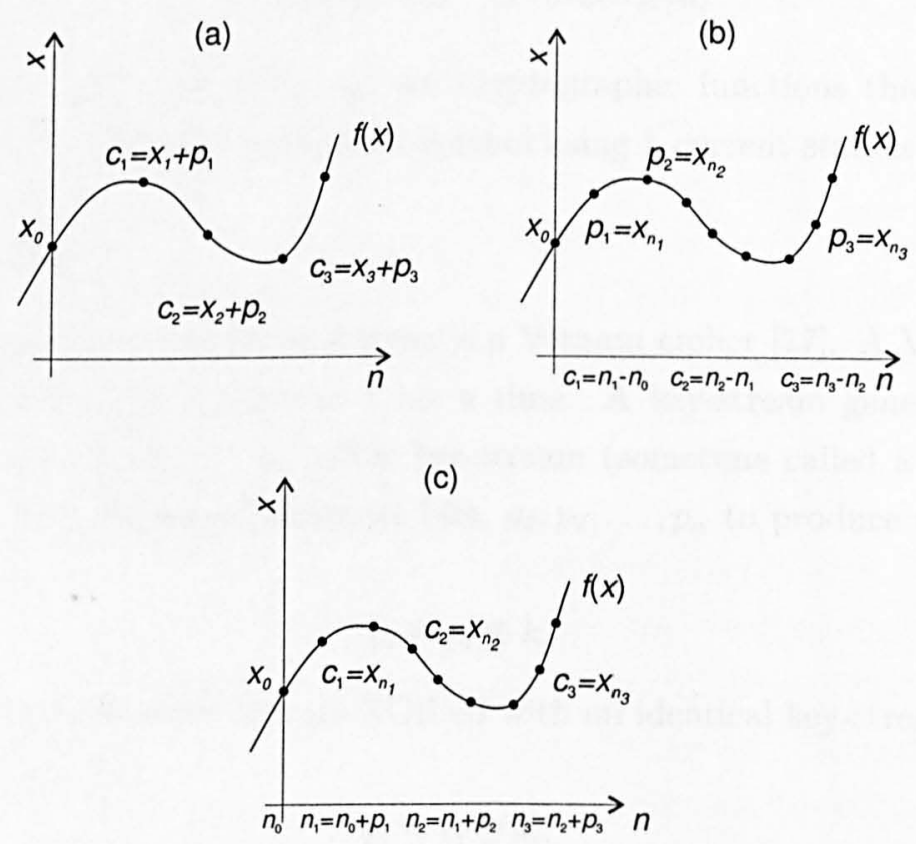

Figure 2.4: Trajectories of stream ciphers based on a single trajectory. (a) The ciphertext $c$ is the plaintext $p$ plus chaotic noise $x$ (e.g. simple XOR cipher using chaos key stream suggested by Bianco, see Section 4.2.3); (b) The ciphertext $c$ is a number of iterations $n$ (Baptista, Section 4.2.7); (c) The ciphertext $c$ is the system state after $p$ iterations (Gallagher, Section 4.2.7). 
to each other, for example, in the American Data Encryption Standard (DES) developed at IBM or in its successor, the Advanced Encryption Standard (AES).

2. Stream ciphers are more diverse from the view point of using the trajectory (Figure $2.4 \mathrm{a}-\mathrm{c}$ ). The main difference from block ciphers is that all the ciphertext is 'cohered' with one or more trajectories and the encryption transformation each block depends on the system state $x$. The trajectory length is not limited and depends on the message length. Chapter 4 provides example ciphers for each type $a, b$ and $c$. Simplifying, we can write

$$
\begin{gathered}
E\left(p_{n}, k\right)=g\left(f^{n}\left(x_{n}, k\right), p_{n}\right), \\
D\left(c_{n}, k\right)=g^{-1}\left(f^{n}\left(x_{n}, k\right), c_{n}\right)
\end{gathered}
$$

where $g\left(x, p_{n}\right)$ and $g^{-1}\left(x, c_{n}\right)$ are cryptographic functions that respectively encrypts and decrypts the input symbol using a current state $x$ as the key.

\section{Vernam Cipher}

The simplest stream encryption scheme is a Vernam cipher [77]. A Vernam cipher converts plaintext into ciphertext 1 bit a time. A key-stream generator outputs a stream of bits: $k_{1}, k_{2}, \ldots, k_{n}$. This key-stream (sometime called a running key) is XOR'ed with a stream of plaintext bits, $p_{1}, p_{2}, \ldots, p_{n}$ to produce the stream of cipher bits, i.e.

$$
c_{i}=p_{i} \oplus k_{i}
$$

To decrypt, the ciphertext bits are XOR'ed with an identical key-stream to recover the plaintext bits, i.e.

$$
p_{i}=c_{i} \oplus k_{i}
$$

since $p_{i} \oplus k_{i} \oplus k_{i}=p_{i}$.

Figure 2.4 (a) represents a similar encryption scheme. The trajectory corresponds to the key-stream. After each iteration $c_{i}=p_{i}+x_{i}$ (for decryption $p_{i}=c_{i}-x_{i}$ ).

If the key-stream $k=\left\{k_{i}\right\}$ is truly random (has a truly uniform distribution), the Vernam cipher is called one-time pad, which is perfectly secure. The concept of true randomness and perfect security is discussed in Chapter 3. 


\subsubsection{Pseudo-Random Number Generators}

In practical cryptography, one-time pads are not used since it is technically difficult to generate and distribute enormous truly random keys. Random pads are not reproducible. Pseudo-Random (Number) Generators (PRNG) extract a compact seed into a long sequence. The output pseudo-random sequences appear random, i. e. the probability distribution function cannot be efficiently distinguish from that of white noise.

PRNG's play a central role in computer cryptography. Although they may be applied explicitly in stream ciphers, the main application is round key generation in block encryption schemes.

According to our approach, we consider PRNG's as a nonlinear dynamic system that differs from the conventional approach in which pseudo-random numbers are generated from mod-based iterations (e.g. $x_{n+1}=r x_{n} \bmod q$ as illustrated in Section 3.6). Figure 2.1 represents a typical PRNG producing a sequence $x_{1}, x_{2}, \ldots$ from the seed given by the initial condition $x_{0}$ and parameter $p$.

The critical requirements for dynamic systems to be used in cryptography are pseudo-randomness and unpredictability. Chapter 3 provides formal definitions of these properties and links them to concepts of chaotic dynamics.

\subsubsection{Confusion and Diffusion}

Using pseudo-random sequences, modern algorithms are far from the theoretical perfection and thus retain some information about the plaintext in the ciphertext. Roughly speaking, the randomness or unpredictability of the seed is spread through the whole pseudo-random sequence. An amount of plaintext redundancy is still invariant after encryption (kept in the ciphertext), which makes possible both knownplaintext and ciphertext-only attacks. To avoid this (at least to some degree), the plaintext should be reduced as close as possible to its true entropy by means of a good compression algorithm. If ideal compression could be achieved, then changing any number of bits in the compressed message would result in another sensible message when uncompressed (see also Section 3.2.3). Otherwise, two basic techniques for hiding redundancies in ciphertext should be used (Shannon, [92]): 


\section{Confusion}

Confusion ensures that the (statistical) properties of plaintext blocks are not reflected in the corresponding ciphertext blocks. Instead every ciphertext must have a pseudo-random appearance to any observer or standard statistical test.

\section{Diffusion}

In terms of plaintexts, diffusion demands that (statistically) similar plaintexts do result in completely different ciphertexts even when encrypted with the same key. In particular, this requires that any element of the input block influences every element of the output block in a complex irregular fashion.

In terms of a key, diffusion demands that similar keys do result in completely different ciphertexts even when used for encrypting the same block of plaintext. This requires that any element of the key influences every element of the output block in a complex irregular fashion. Additionally, this property must also be valid for the decryption process because otherwise an intruder might recover parts of the input block from an observed output by a partly correct guess of the key used for encryption.

Most block ciphers explicitly implement confusion and diffusion: respectively, their iterated function consists of substitution and permutation phases. Eventually, both phases taken together, ensure a highly nonlinear transformation resulting in a pseudo-random and unpredictable ciphertext.

\subsubsection{Cryptanalysis}

The whole point of cryptography is to keep the plaintext (or the key, or both) secret from eavesdroppers (also called adversaries, attackers, interceptors, interlopers, intruders, opponents, or simply the enemy). Eavesdroppers are assumed to have complete access to the communication between the sender and receiver.

Cryptanalysis is the science of recovering the plaintext of a message without access to the key. Successful cryptanalysis may recover the plaintext or the key. It also may find weaknesses in a cryptographic system that eventually leads to recovery of the plaintext or key. (The loss of a key though non-cryptanalytic means is called a compromise.) 
A fundamental assumption in cryptanalysis (first enunciated by the Dutchman A Kerckhoff) assumes that the cryptanalyst has complete details of the cryptographic algorithm and implementation. Some old cryptographic systems rely on the secrecy of the algorithms; such algorithms are only of historical interest and are not adequate for real-world needs. All modern algorithms use a key to control encryption and decryption ("The enemy knows the system being used" as stated by Shannon); a message can be decrypted only if the key matches the encryption key.

An attempted cryptanalysis is called an attack. There a four principal types of cryptanalytic attacks; each of them assumes that the cryptanalyst has complete knowledge of the encryption algorithm used:

Cyphertext-only attack The cryptanalyst has the cyphertext of several messages, all of which have been encrypted using the same encryption algorithm. The cryptanalyst's job is to recover the plaintext of as many messages as possible, or to deduce the key (or keys) used to encrypt the messages, in order to decrypt other messages encrypted with the same keys.

Known-plaintext attack The cryptanalyst not only has access to the cyphertext of several messages, but also to the plaintext of those messages. The problem is to deduce the key (or keys) used to encrypt the messages or an algorithm to decrypt any new messages encrypted with the same key (or keys).

Chosen-plaintext attack The cryptanalyst not only has access to the cyphertext and associated plaintext for several messages, but also chooses the plaintext that gets encrypted. This is more powerful than a known-plaintext attack, because the cryptanalyst can choose specific plaintext blocks to encrypt those that might yield more information about the key. The problem is to deduce the key (or keys) used to encrypt the messages or an algorithm to decrypt any new messages encrypted with the same key (or keys).

Adaptive-chosen-plaintext attack This is a special case of a chosen-plaintext attack. Not only can the cryptanalyst choose the plaintext that is encrypted, but can also modify the choice based on the results of previous encryption. In a chosen-plaintext attack, a cryptanalyst might just be able to choose one large 
block of plaintext to be encrypted; in an adaptive-chosen-plaintext attack it is possible to choose a smaller block of plaintext and then choose another based on the results of the first, and so on.

In addition to the above, there are at least three other types of cryptanalytic attack.

Chosen-cyphertext attack The cryptanalyst can choose different cypher-texts to be decrypted and has access to the decrypted plaintext. For example, the cryptanalyst has access to a tamperproof box that does automatic decryption. The problem is to deduce the key. This attack is primarily applicable to publickey algorithms. A chosen-cyphertext attack is sometimes effective against a symmetric algorithm as well. (A chosen-plaintext attack and a chosencyphertext attack are together known as a chosen-text attack).

Chosen-key attack This attack does not mean that the cryptanalyst can choose the key; it means that there is some knowledge about the relationship between different keys - it is a rather obscure attack and not very practical.

\subsection{Chaotic Dynamics}

\subsubsection{Dynamic Systems}

A dynamic continuous-state continuous-time system $\mathcal{S}=\langle X, \mathcal{K}, f\rangle$ depending on parameters can be considered in the following form

$$
\frac{d x}{d t}=f(x, k), \quad x \in X \subseteq R^{d}, k \in \mathcal{K} \subseteq R^{d_{K}},
$$

where $f: X \times \mathcal{K} \rightarrow Y$ is a smooth vector function, $X$ is a state space, and $\mathcal{K}$ is a parameter space. The equation satisfies the conditions of the existence and uniqueness of solutions $x\left(t, x_{0}\right)$ with the initial condition $x_{0}=x\left(0, x_{0}\right)[4,11]$. The solution curve $x\left(t, x_{0}\right)$, is called the trajectory.

In cryptography, we focus on a dynamic discrete-time system, which can be written in the following form:

$$
x_{n+1}=f\left(x_{n}, k\right), \quad x_{n} \in X \subseteq R^{d}, k \in \mathcal{K} \subseteq R^{d_{K}}, n=0,1,2, \ldots
$$


where $x_{i}$ is a discrete state of the system. The trajectory $\varphi\left(i, x_{0}\right)$ is defined by the sequence $x_{0}, x_{1}, x_{2}, \ldots$ Clearly, equation (2.2) is similar to a cryptographic iterated function, used in PRNG, block ciphers and other constructions (see Figures 2.12.4). Consequently, in both nonlinear dynamics and cryptography we deal with an iterated key-dependent transformation of information.

\subsubsection{Chaotic Systems}

There are several sufficient conditions satisfied by a dynamic system to guarantee chaos [29]; the sensitivity to initial conditions and topological transitivity are the most common.

Definition 2 (chaotic dynamic system, [31, 79, 29, 4]) A chaotic continuous-state discrete-time system (continuous chaos for short) is a dynamic system $\mathcal{S}=\langle X, f\rangle$ with two properties:

1. Given a metric space $X \subseteq R^{N}$ and a mapping $f: X \rightarrow X$, we say that $f$ is topological transitive on $X$ if for any two open sets $U, V \subset X$, there is $n \geq 0$ such that $f^{n}(U) \cap V \neq \emptyset$.

2. The map $f$ is said to be sensitive to initial conditions if there is $\delta>0, n \geq 0$ such that for any $x \in X$ and for any neighborhood $H_{x}$ of $x$ there is $y \in H_{x}$, such that $\left|f^{n}(x)-f^{n}(y)\right|>\delta$.

This can be interpreted in natural language as follows: a dynamic system is chaotic if all trajectories are bounded and nearby trajectories diverge exponentially at every point of the phase space (see Figure $2.5^{1}$ ). A synergy between chaotic and cryptographic systems section 2.1 can be described as follows:

\footnotetext{
${ }^{1}$ Trajectories illustrated in Figures 2.5 are continuous and belong to a two dimensional system said to be chaotic. This diagram should be interpreted only as an example to be extended in more dimensions. Clearly, a 2D dynamic system with continuous trajectories cannot be chaotic (otherwise any interception of trajectories will lead to periodical cycles) unlike 3 or more dimensional system. This note should not be applied to discrete-time system (2.2) where chaotic behavior is possible in 1 or 2 dimensions.
} 
(a)

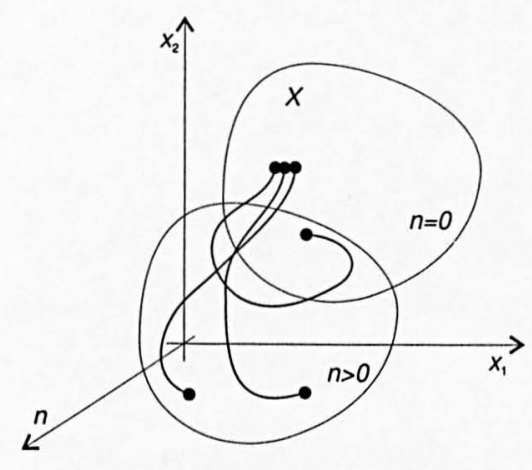

(b)

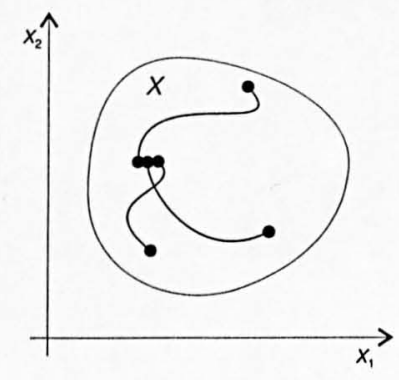

Figure 2.5: A two-dimensional chaotic system: (a) Time space; (b) Phase space.

The topological transitivity ensures that the system output covers all the state space e.g. any plaintext can be encrypted into any ciphertext.

The sensitivity to the initial condition corresponds to Shannon's requirements for an encryption system (section 2.1.5). In both chaos and cryptography we are dealing with systems in which " a small variation of any variable changes the outputs considerably" [92].

\subsubsection{Attractors}

Most nonlinear systems are unpredictable and yet patterned, it is called strange and since it tends to produce a fractal ${ }^{2}$ geometric shape. It is said to be attracted to that shape. Tsonis defines attractor as "a limit set that collects trajectories" [96]. When we observe the dynamic behaviour of the state point in the state space we can notice that trajectories tend to contract in certain areas; this contraction is called the attractor. The attractor is actually a set of points such that all trajectories

\footnotetext{
${ }^{2}$ The term fractal was coined by Benoit Mandelbrot in 1975. It comes from the Latin fractus, meaning an irregular surface like that of a broken stone. Fractals are non-regular geometric shapes that have the same degree of non-regularity on all scales. "Fractal Geometry plays two roles. It is the geometry of deterministic chaos and it can also describe the geometry of mountains, clouds and galaxies"-Benoit Mandelbrot.
} 
nearby converge to it. At the same time, by definition, nearby trajectories diverge exponentially at every point. The coexistence of these two properties is another reason for the 'strange name' of the attractor.

Chaos is centered on the concept of the strange attractor. If we watch the flow of water from a faucet we turn, so the water goes faster and faster; we will see activity from smooth delivery to gushing states. These various kinds of flow represent different patterns to which the flow is attracted.

The four kinds of attractors are:

1. Point attractor, such as a pendulum swinging back and forth and eventually stopping at a point. The attractor may come as a point, in which case, it gives a steady state where no change is made.

2. Periodic attractor, just add a mainspring to the pendulum to compensate for friction and the pendulum now has a limited cycle in its phase space. The periodic attractor portrays processes that repeat themselves.

3. Torus attractor, picture walking on a large doughnut, going over, under and around its outside surface area, circling, but never repeating exactly the same path you went before. The torus attractor depicts processes that stay in a confined area but wander from place to place in that area.

4. Strange attractor, this attractor deals with the three-body problem of stability. The strange attractor shows processes that are stable, confined and yet never do the same thing twice. Three non-linear equation solutions exhibit a fractal structure in computer simulations of the strange attractor. In other words, each solution curve tended to the same area, the attractor area, and cycled around randomly without any particular set number of times, never crossing itself, staying in the same phase space, and displaying self-similarity at any scale. The operative term here is self-similarity. Each event, each process, each period, each end-state in phase-space is never precisely identical to another; it is similar but not identical.The attractor acts on the system as a whole and collects the trajectories of perturbation in the environment. Though these systems are unstable, they have patterned order and boundary. 
The term 'attractor' has no counterpart in cryptography, precisely, cryptography attempts to hide the attractor and any other recognizable structure of the system, making it unpredictable.

\subsubsection{Lyapunov Exponents}

Definition 2 introduced the concept of the sensitivity to the initial condition. The Lyapunov exponent, denoted by $\lambda\left(x_{0}\right)$, is a quantitative measure of the exponential divergence of trajectories starting from the neighborhood of $x_{0}[16]$. For a onedimensional system

$$
\left|f^{n}\left(x_{0}+\varepsilon\right)-f^{n}\left(x_{0}\right)\right|=\varepsilon \cdot e^{n \lambda\left(x_{0}\right)},
$$

where $\varepsilon$ is a small perturbation from the initial condition $x_{0}$ and $n$ is the number of iterations (the discrete time). Generally, $\lambda$ depends on the initial condition, so we can estimate its average value. In a measure-preserving system (2.2.6) $\lambda$ is constant for all trajectories. The Lyapunov exponent is given by the limit

$$
\lambda\left(x_{0}\right)=\lim _{n \rightarrow \infty} \lim _{\varepsilon \rightarrow 0} \frac{1}{n} \log \left|\frac{f^{n}\left(x_{0}+\varepsilon\right)-f^{n}\left(x_{0}\right)}{\varepsilon}\right|
$$

or

$$
\lambda\left(x_{0}\right)=\lim _{n \rightarrow \infty} \frac{1}{n} \sum_{k=1}^{n} \log \left|f^{\prime}\left(x_{k}\right)\right|=\lim _{n \rightarrow \infty} \frac{1}{n} \log \prod_{k=1}^{n}\left|f^{\prime}\left(x_{k}\right)\right|
$$

For each $k, f^{\prime}\left(x_{k}\right)$ tells us how much the function $f$ is changing with respect to its argument at the point $x_{k}$. This derivative expresses the magnitude of change in the transition from $x_{k}$ to $x_{k+1}$. The limit of the average of the derivative logarithms over $n$ iterations is taken to provide a measure of how fast the orbit changes as (discrete) time propagates. A positive Lyapunov exponent is an indication of chaotic behavior.

For a $d$-dimensional system we have a set $\lambda=\left\{\lambda_{1}, \ldots, \lambda_{d}\right\}$ and more complex behavior, but still qualitatively the same as the one dimensional case [78].

A more accurate measure is the Kolmogorov-Sinai (KS) entropy because it considers the resolution (the precision) under which the system is observed. Chapter 3 studies the complexity of chaotic systems using the KS entropy.

From the view point of cryptography, $\lambda$ measures the encryption efficiency. Larger $\lambda$ indicates that fewer cryptography transformation will be required to reach a 
certain level of the ciphertext unpredictability. Chapter 4 provides numeric values of $\lambda$ for certain chaos-based encryption systems.

\subsubsection{Bifurcation}

The term bifurcation (from Latin bifurcus - two pronged) usually means a qualitative transformation from regular to chaotic behavior as a control parameter is smoothly varied. There are several types of bifurcation; the best known is period doubling discovered by Feighenbaum (see Figure 4.9). In bifurcation points, the number of equilibrium states doubles. As the control parameter increases, this happens more often, finally, when the number of possible states is infinitely large, the system becomes chaotic.

Certain cryptographic applications use control parameters as a key. Clearly, the key space should correspond to chaotic behavior. In a good encryption system, the number of states and the length of orbits should not depend on the choice of parameters.

\subsubsection{Ergodic and Mixing Systems}

Let the dynamic system $\mathcal{S}=\langle X, f\rangle$ have a $f$-invariant measure $\mu[8], \mu(X)<\infty$, that is

$$
\forall A \in \sigma(X), \quad \mu(A)=\mu\left(f^{-1}(A)\right),
$$

where $\sigma(X)$ is the $\sigma$-algebra of measurable subsets of $X$. Assume the $f$-invariant measure is equivalent to the Lebesgue with the density function $g(x)$ bounded with some positive constants $g_{1}$ and $g_{2}$ :

$$
0<g_{1}<g(x)<g_{2}
$$

where $\forall A \in \sigma(X), \quad \mu(A)=\int_{A} g(x) d x$. If $g_{1}$ is close to $g_{2}$ then the measure $\mu$ is close to the uniform distribution.

A dynamic system $\mathcal{S}=\langle X, f\rangle$ is ergodic if it has only trivial invariant sets, i.e. if and only if either $\mu(A)=0$ or $\mu(X \backslash A)=0$, whenever $A$ is a measurable, invariant under $f$, subset of the space $X$ (the invariance of $A$ means $f(A) \subset A$ ). Ergodicity implies that the space $X$ cannot be divided into invariant nontrivial (with respect to 
the measure $\mu$ ) disjoint parts (in the case of smaller disjoint parts, any brute force attack is restricted to one part of the partition i.e. an intruder will have to search through the whole state space $X)$ [95].

A dynamical system $\mathcal{S}=\langle X, f\rangle$ is mixing if it satisfies the condition

$$
\forall C, P \in \sigma(X), \quad \lim _{n \rightarrow \infty}\left(\frac{\mu\left(f^{-n}(C) \cap P\right)}{\mu(P)}\right)=\frac{\mu(C)}{\mu(X)} .
$$

If $\mu(X)=1$ (the measure $\mu$ is probabilistic) we can write

$$
\lim _{n \rightarrow \infty}\left(\mu\left(f^{-n}(C) \cap P\right)\right)=\mu(C) \mu(P) .
$$

The mixing property implies that the part of $P$, which after $n$ iterations of $f$ is contained in $C$ is asymptotically proportional to the volume (in the sense of the measure $\mu$ ) of $C$ in $X$. Moreover, the iterations of $f$ make each set $C$ statistically independent from $P$ (asymptotically). In other words, the trajectory starting at a fixed point $x_{0} \in X$, after $n$ iterations reaches any region with the same probability. Vice versa, for a fixed final state $x_{n}$ and sufficiently large $n$, any state $x_{0}$ is $\mu$ equiprobable [68] (see also section 3.6).

\subsubsection{Binary Chaos}

We have discussed the main properties of chaos defined on real numbers. However, digital cryptography is based on discrete state systems.

In 1998 Waelbroeck and Zertuche [97] proposed a theory of deterministic chaos for binary systems. Binary systems, like a cellular automata and neural networks, are the best known class of chaotic discrete systems. Consider a state space

$$
\Omega=\left\{\alpha \mid \alpha \in \mathbb{Z}_{2}^{*}\right\}, \quad \mathbb{Z}_{2}=\{0,1\}
$$

The system state $\alpha \in \Omega$ is given by a sequence of symbols (bits):

$$
\alpha=\alpha(1) \alpha(2) \ldots \alpha(n) \ldots
$$

A natural topological measure for binary systems is the Hamming distance defined as

$$
d_{H}\left(\alpha, \alpha^{\prime}\right) \equiv \sum_{i=1}^{n}\left|\alpha(i)-\alpha^{\prime}(i)\right|
$$


(a)

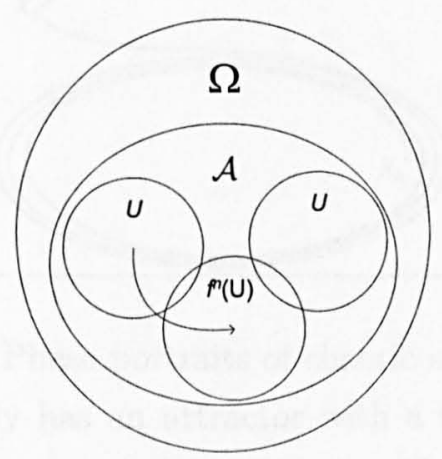

(b)

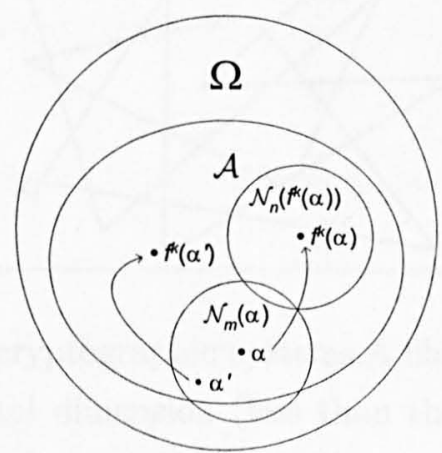

Figure 2.6: Binary Chaos: the sensitivity to the initial condition (a) and the topological transitivity (b) are defined on the space $\Omega$.

i. e. $d_{H}$ is a number of bits by which the two strings $\alpha$ and $\alpha^{\prime}$ differ. However, in the limit $n \rightarrow \infty$ (infinite strings) the Hamming distance cannot define the topology of $\Omega$. For this case we can introduce a topological base

$$
\mathcal{N}_{n}(\alpha)=\left\{\alpha^{\prime} \in \Omega \mid \alpha(i)=\alpha^{\prime}(i), \forall i<n\right\},
$$

where $n=1,2,3 \ldots$. Now we can generalize the definition of chaos from $R^{d}$ to $\Omega$ :

Definition 3 (binary chaos, [97]) Let $\mathcal{A} \subset \Omega$ and $f: \mathcal{A} \rightarrow \mathcal{A}$. The binary system $\langle\mathcal{A}, f\rangle$ is said chaotic, if two following conditions are satisfied:

1. The function $f$ is topological transitive on $\mathcal{A}$, i. e. for all open subsets $U, V \subset \mathcal{A}$ it exists $n \in \mathbb{Z}$ such as $f^{n}(U) \cap V \neq \emptyset$ (Figure 2.6-b) ${ }^{3}$.

2. The function $f$ is sensitive to the initial conditions on $\mathcal{A}$, i. e. for all $\alpha \in \mathcal{A}$ and $\mathcal{N}_{m}(\alpha)$ it exists $n, k \in \mathbb{N}$ and $\alpha^{\prime} \in\left(\mathcal{N}_{m}(\alpha) \cap \mathcal{A}\right)$, such as $f^{k}\left(\alpha^{\prime}\right) \notin \mathcal{N}_{n}\left(f^{k}(\alpha)\right)$ (Figure 2.6-a).

Hence, a chaotic binary system is defined in a similar way as real space chaos. Section 4.3 discusses the Lyapunov exponent for finite state systems, and Sections 3.6 and 4.3.5 show that conventual cryptographic schemes can be considered as 'limited' binary chaos.

\footnotetext{
${ }^{3}$ If $n<0$ and $f$ is not invertible, we assume $f^{n}(U)=\left\{\alpha \in \mathcal{A} \mid f^{-n}(\alpha) \in U\right\}$.
} 

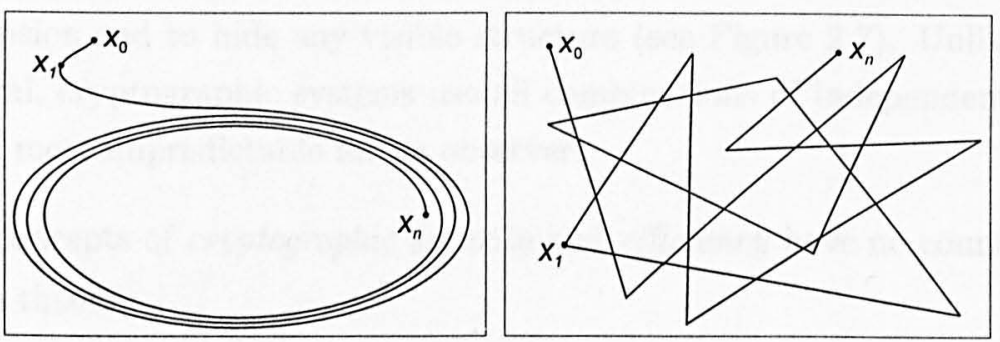

Figure 2.7: Phase portraits of chaotic and cryptographic systems.A chaotic system (left) usually has an attractor with a fractal dimension (less than the number of independent variables). A cryptographic system (right) attempts to maximize the dimension up to an integer.

\subsection{Relationship between Chaos and Cryptography}

So far, we have considered cryptographic systems from the view point of synergetics and chaotic dynamics. Clearly, chaos theory and cryptography studies a nonlinear iterative transformation depending on parameters. Such properties as sensitivity to initial conditions, exponential divergence of trajectories and mixing can be applied in both areas.

In terms of the object of study the following differences are important:

1. Chaos theory studies nonlinear systems defined on an infinite state space (e.g vectors of real numbers or infinite binary strings), whereas cryptography relies on a finite-state machine (computer). All chaos models implemented on a computer are approximations, i. e. pseudo-chaos.

2. Chaos theory studies the asymptotic behavior of a nonlinear system $(n \rightarrow \infty)$, whereas cryptography focuses on the effect of a small number of iterations $(n \ll \infty)$. For example, $n=16$ in a block cipher and $n \sim$ [message size] in a stream cipher.

3. Classical chaotic systems have visually recognizable attractors (the phase space portrait of the system) whose dimension is fractional ${ }^{4}$. Cryptography attempts to maximize the attractor dimension to an integer, the topological

\footnotetext{
${ }^{4}$ We say a set is $n$-dimensional if we need $n$ independent variables to exactly identify any state
} 
dimension, and to hide any visible structure (see Figure 2.7). Unlike chaos in general, cryptographic systems use all combinations of independent variables to be most unpredictable for an observer.

4. The concepts of cryptographic security and efficiency have no counterparts in chaos theory.

The following table summarizes the relationship between the concepts.

Chaos theory
chaotic system
— nonlinear transformation
— infinite number of states
— infinite number of iterations
initial condition
final state
initial conditions and parameters
asymptotic independence of the initial
and final states
the sensitivity to the initial condition
and parameters, mixing

\section{Cryptography}

pseudo-chaotic system

- nonlinear transformation

- finite number of states

- finite number of iterations

plaintext

ciphertext

key

confusion

It is natural to suggest using known chaotic systems for the purpose of encryption. Section 3.6 provides a theoretical construction of a chaos-based PRNG and Chapter 4 is devoted to its computer implementations (pseudo-chaos).

of the system. This notion of dimension is called the topological dimension of a set. Fractal objects, in particular, chaotic attractors, can have less degrees of freedom than its topological dimension because not all combinations of independent variables are possible. For example, a geometrical fractal plotted on a $2 \mathrm{D}$ plane might have the dimension greater than 1 (corresponding to line) and less than 2 (corresponding to surface). 


\section{Chapter 3}

\section{Randomness, \\ Complexity and Chaos.}

The concepts of randomness, unpredictability, complexity and entropy form the basis of modern cryptography. Mathematically, the design of a cryptosystem can be interpreted as the design of a key-dependent bijective transformation, most unpredictable for an observer with a given amount of resources.

The present chapter links these concepts with chaotic dynamics and suggests a pseudo-random generator based on a chaotic system.

\subsection{Informal Overview}

In studying a cryptosystem (PRNG, encryption algorithm or key exchange scheme), a cryptanalyst has access to time series of this dynamic system and knows the iterated function (the algorithm). However, the time series is not a compact subset of the trajectory (intermediate states are hidden) and the iterated function has a secret parameter (the key). We can think of the sample as being 'random', 'unpredictable' and 'complex'. What do these properties mean mathematically? How do they relate to chaos? This chapter attempts to give an answer.

Perfect security is achieved when the cryptosystem is absolutely unpredictable for an external observer, i.e. all possible outcomes (states, sub-trajectories) are equiprobable and do not depend on the previous states. In other words, the state 


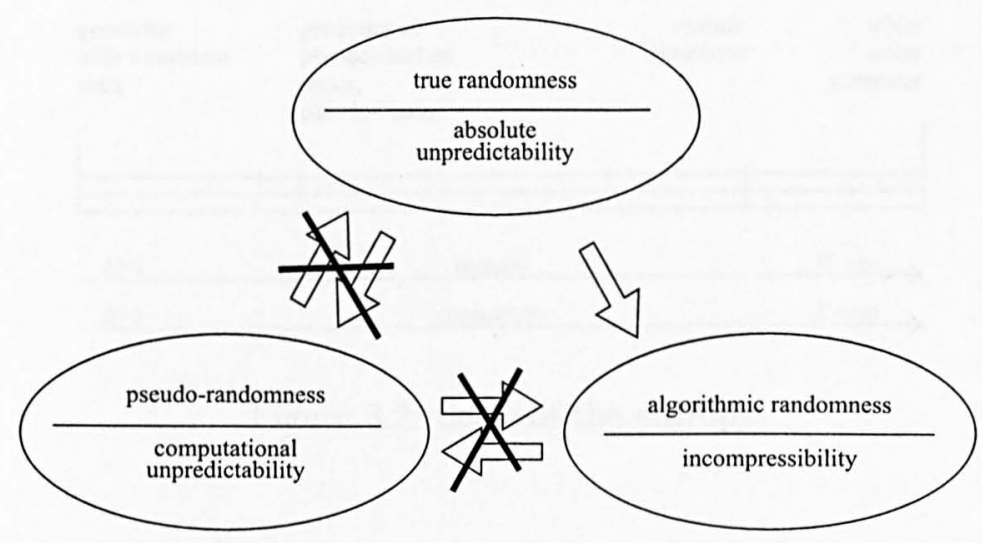

Figure 3.1: Relationship between the concepts.

sequence has a uniform probability distribution and no correlations (patterns). The concept of absolute unpredictability is equivalent to the true randomness and related to the white noise. A source of the white noise can be a highly dimensional system (e. g. microscopic motion in the ideal gas).

In the real world, cryptographic systems provide a certain level of practical security that is much 'lower' that the perfect security (due to usability and cost reasons). At this level we deal with 'pseudo' concepts. Pseudo-random sequences cannot be efficiently distinguished from the uniform noise by known algorithms. Similarly, computationally unpredictable sequences cannot be predicted with the available computer resources. It is possible to show that the pseudo-random sequence (cryptosystem) is computationally unpredictable, and vice versa.

Together with probabilistic properties we shall consider the algorithmic complexity, i.e. the length of the shortest algorithm producing the cryptographic sequence. Intuitively, the (internal) complexity of the system provides the (external) unpredictability. A sequence is called algorithmically random, if its algorithmic complexity equals the length of the sequence. An algorithmically random sequence is computationally incompressible and contains no recognizable patterns (redundancies).

Clearly, a purely random system is also algorithmically random (Figure 3.1). The concepts of pseudo and algorithmic randomness are different: a pseudo-random 


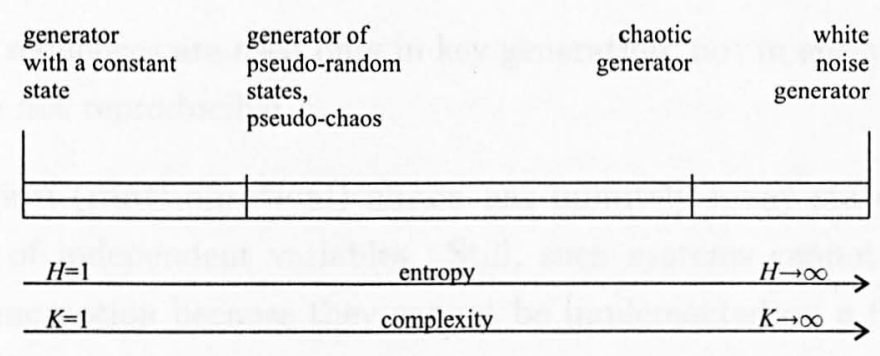

Figure 3.2: Scale of the entropy

string is generated with a compact seed, but the external observer is not able (practically) to reconstruct the generator and predict the sequence. In other words, the string is highly compressible for authorized communication parties, but computationally incompressible for the adversary. In the general case, an algorithmic random string can be predicted by a probabilistic machine.

Randomness or unpredictability can be 'measured' using such properties as the algorithmic complexity or the entropy i.e. the degree of our uncertainty about the system. Quantitatively, the Shannon entropy is in direct proportion to the algorithmic complexity (in ergodic systems, where statistical properties of a single sequence coincides with that of all sequences, emitted by a generator). A randomness measure for chaos is the Kolmogorov-Sinai entropy that is, roughly speaking, a multiresolution integration of Lyapunov exponents.

A 'fully predictable' system has a well-know state, its complexity is 1 . A fully unpredictable system (the uncorrelated white noise) is an infinite source of information and infinite complexity and entropy (Figure 3.2). Cryptographic systems are somewhere in-between: they are complex enough to be unpredictable by an external observer, but not too much to be reproducible.

The following types of chaos can be considered in the context of cryptography:

Natural chaos (e.g. the environment) is a highly dimensional system with infinitely many states and independent variables. Generally, the entropy of such a system is not maximal because of its self-organization and correlations. There are cryptographic applications using natural chaos; for example, Intel's hardware RNG captures randomness from the thermal noise of the computer. Such 
random sequences are used only in key generation, not in encryption, because they are not reproducible.

Low-dimension (mathematical) chaos has infinitely many states and a small number of independent variables. Still, such systems cannot be applied to digital encryption because they cannot be implemented on a finite-state machine. This chapter discusses the cryptographic properties of mathematical chaos and studies a chaos-based PRNG with infinite states. In the following chapter we attempt to transform the results into different types of approximations (pseudo-chaos).

\subsection{Complexity Theory Approach}

\subsubsection{Turing Machine}

Throughout this thesis we will use the common terminology from complexity theory $[5,73]$. For the sake of self-sufficiency we provide a brief introduction to the subject.

A Turing machine is a hypothetical device that can theoretically implement any computer algorithm. It provides a unified framework to measure the complexity (i.e. program length and working time) of computational problems such as generating, transforming and matching cryptographic sequences.

A basic model of a Turing machine consists of (1) a semi-infinite tape divided into cells, (2) a read-write head (3) a control unit, which is a finite-state sequential machine. We denote a Turing machine as

$$
T=\left\langle S, \mathcal{A}, \Gamma, F, q_{0}\right\rangle
$$

where $S$ is the finite state set of the control; $\mathcal{A}$ is the finite tape alphabet $(\mathcal{A}=$ $\{0,1\}) ; \Gamma$ is a finite rule state of the form $\gamma: S \times \mathcal{A} \rightarrow S \times \mathcal{A} \times\{L, N, R\} ; F \subseteq S$ is a set of halting accepting states; and $q_{0} \in S$ is the initial state. The state $\{L, N, R\}$ includes tape commands: 'move left' $(L)$, 'stay in place' $(N)$ and 'move right' $(P)$. The machine configuration $T$ is the triplet $\langle s, \alpha, i\rangle$, where $s \in S$ is the current state, $\alpha \in \mathcal{A}^{*}$ the tape string, and $1 \leq i \leq|\alpha|$ is the head position counting from the left end of the tape. 
The machine is initialized in the following way: (1) a string $\alpha \subset \mathcal{A}^{*}$ is loaded onto the tape; (2) the head is seeded to the leftmost position; and (3) the initial state $q$ is assigned to the state variable $s$. In every step, (1) the machine reads a symbol from the current cell, depending on the symbol read and the current state, (2) it makes a transition to a new state; (2) it overwrites the current cell with a new symbol; (3) it moves the head one step left or right, or stays in place. The machine halts when a state $f \in F$ is reached.

A Turing machine is said to accept a string $\alpha$ if a sequences of rules $\gamma_{1}, \ldots, \gamma_{m} \subset$ $\Gamma^{*}$ exists that puts the machine from the initial state $s_{0}$ to any halting accepting state $f \in F$. The machine rejects a string, if it halts in $s \notin F$ or if it never halts.

Turing machines may take several other forms: (a) The tape may be extended to make it doubly infinite; (b) One or more additional tapes may be added; (c) The storage medium may be the plane ruled into squares or $n$-dimensional space ruled into $n$-dimensional cubes. It can be shown that each of these extensions of the basic machine are equivalent in the sense that a basic machine can be constructed that 'simulates' the behavior of the extended machine.

A language $\mathcal{L}$ over a finite alphabet $\mathcal{A}$ is a subset $(A)^{\star}$, i.e. a subset of all finite strings over $\mathcal{A}$. A machine is said to accept a language $\mathcal{L}$ if it accepts all the strings $\alpha \in \mathcal{L}$ and rejects $\beta \notin \mathcal{L}$.

A deterministic Turing machine has single-valued rules $\gamma: S \times \mathcal{A} \rightarrow S \times \mathcal{A} \times$ $\{L, N, R\}$, i.e. there are no rules with the same left parts $S \times \mathcal{A}$. By contrast, nondeterministic machines may have multi-valued rules.

If there exists a polynomial $p(l)$, limiting the machine working time $m$ (the number of steps) depending of the input string length $l(m<p(l))$, the machine is said to run in polynomial time. The complexity class $\mathbf{P}$ is the set of languages accepted by deterministic polynomial-time machines. The complexity class NP is the set of languages accepted by nondeterministic polynomial-time machines.

A Probabilistic Turing machine is a deterministic Turing machine that can flip a fair coin to determine its next move. A probabilistic machine is said to accept a language $\mathcal{L}$ if it enters an accepting state for $\alpha \in \mathcal{L}$ with the probability $p_{1}>2 / 3$ and it halts $\alpha \notin \mathcal{L}$ with the probability $p_{0}>2 / 3$.

The complexity class BPP consists of all languages recognized by probabilistic 
polynomial-time machines.

\subsubsection{Algorithmic Complexity}

The concept of algorithmic complexity was suggested independently by three mathematicians A. N. Kolmogorov, P. Solomonov and G. J. Chaitin:

Definition 4 The algorithmic complexity $K_{M}(\alpha)$ of a finite string $\alpha \in\{0,1\}^{n}$ with respect to a Turing Machine $M$ is the length $l(\pi)$ of the smallest computer program $\pi$, which generate it, that is

$$
K_{M}(\alpha)=\min _{\pi: M(\pi)=\alpha} l(\pi) .
$$

Kolmogorov showed, that there exists a universal Turing machine $U$, that performs computations equivalent to $\pi$ (designed for an arbitrary machine $M$ ), and the changes of $\pi$ required to adopt it for $U$ depend on $M$ but not on $\alpha$. Consequently, the algorithmic complexity $K_{M}$ with respect to any machine $M$ is related to $K_{U}(S)$ by

$$
K_{U}(S) \leq K_{A}(S)+C_{A},
$$

where $C_{M}$ is a constant, which is independent from $\alpha$ [21]. Hereafter we omit the subscript $U$ assuming $K(\alpha)=K_{U}(\alpha)$.

Unfortunately, algorithmic complexity cannot be commuted i.e. there is no universal solution for simplifying programs and for proving that the length is minimal. We cannot apply this definition directly to compare the complexity of cryptographic sequences or algorithms. Nevertheless the theoretical applications are very important. In particular, the Kolmogorov complexity provides a unified approach to the problem of data compressibility.

\subsubsection{Compressibility and Algorithmic Randomness}

A string $\alpha_{n}$ of length $n$ is said to be $c$-incompressible if $K\left(\alpha_{n}\right) \geq n-c$. Incompressible strings ( $c=0$ or relatively small) are called algorithmically random. 


\subsubsection{Symbolic Complexity}

For an infinite string $\alpha_{\infty}$ or a generator, it is interesting to consider the symbolic complexity given by the limit

$$
c\left(\alpha_{\infty}\right)=\lim _{n \rightarrow \infty} \frac{K\left(\alpha_{n}\right)}{n} .
$$

From (3.1) it follows that the symbolic complexity $c\left(\alpha_{\infty}\right)$ is invariant with respect to the choice of Turing machine. If a string has a finite Kolmogorov complexity (e. g. a pseudo-random string), its symbolic complexity tends to 0 . A truly random string has $c=1$ because its length equals the length of the shortest program. Clearly, $c>0$ if and only if the generator has infinite complexity. In chaotic systems, this happens if the complexity of the initial conditions is infinitely large or a certain amount of randomness gets into the system from the environment.

\subsection{Information Theory Approach}

A cryptanalyst considers the cryptosystem as a source of information. In studying statistical properties of the ciphertext, he/she attempts to reconstruct the secret transformation (precisely, secret parameters of a known algorithm). In ideal cryptosystems, the distribution of the ciphertext cannot be differentiated from the uniform noise, and thus provides no useful information for an adversary.

The two major approaches to statistical analysis of cryptographic strings are as follows:

- Studying properties of one particular string leads to complexity theory as introduced in Section 3.2.

- By contrast, information theory founded by Shannon [91] describes the properties of all strings produced by the source (cryptosystem).

In ergodic systems (in which statistical properties of a particular string coincides with that of all strings emitted by a generator) both approach can be unified (see section 3.3.3). 


\subsubsection{True Randomness}

We define a Probability Distribution Function (PDF) as a function from strings $\mathcal{L}=$ $\left\{\alpha_{j}\right\}$ to nonnegative real numbers, i.e. $\operatorname{Pr}: \mathcal{L} \rightarrow[0,1]$ such that $\sum_{\alpha \in \mathcal{L}} \operatorname{Pr}(\alpha)=1$.

Definition 5 A string $\alpha$ is called truly (purely) random (or unpredictable) if for any substrings $\beta_{n}, \gamma_{n} \in \alpha, 0<n<\operatorname{length}(\alpha)$

$$
\operatorname{Pr}\left(\beta_{n}\right)=\operatorname{Pr}\left(\gamma_{n}\right)
$$

A truly random string cannot be predicted, i.e. for any symbol $s_{i} \in \alpha$, the conditional probability $\operatorname{Pr}\left(s_{i} \mid s_{i-1}, s_{i-2}, \ldots\right)=\operatorname{Pr}\left(s_{i}\right)$. In other words, an arbitrary large knowledge about the previous states does not increase the probability of the successful prediction of the next state.

An infinite and truly random string has a delta autocorrelation function, and an infinite and uniform power spectrum (white noise).

\subsubsection{Shannon Entropy}

Claude Shannon generalized the term entropy from thermodynamics to abstract problems in communication and information theories [91]. The entropy measures the amount of information required to determine precisely the system state among all possible states. In other words, it is a relative degree of our uncertainty or the lack of information we have about the exact state of a system. In cryptography, the entropy is related the unpredictability of an encryption system for an adversary.

The entropy of a string $\alpha_{n}$ of the length $n$ is defined as

$$
H_{n}=-\sum_{\alpha \in \mathcal{A}^{n}} \operatorname{Pr}\left(\alpha_{n}\right) \log _{|\mathcal{A}|} \operatorname{Pr}\left(\alpha_{n}\right),
$$

where $\operatorname{Pr}: \mathcal{A}^{n} \rightarrow[0,1]$ is the PDF of $\alpha_{n}$ on the set of $n$-th symbol strings. The maximum of $H_{n}$ is achieved when $\operatorname{Pr}\left(\alpha_{n}\right)$ is a uniform distribution (the string is truly random). 
The conditional entropy $h_{n}$ denotes the average amount of information supplied with each $(n+1)$-th symbol provided the previous $n$ symbols are known:

$$
h_{n}=h_{n+1 \mid n}= \begin{cases}H_{n+1}-H_{n}, & n \geq 1 \\ H_{1}, & n=1\end{cases}
$$

In other words, $h_{n}$ quantifies the average uncertainty when predicting the next symbol. As soon as the knowledge about a previous state cannot increase the uncertainty, the function $H_{n}$ is non decreasing and $h_{n+1} \leq h_{n}$.

For a stationary information source there exists a limit

$$
h_{S h}=\lim _{n \rightarrow \infty} h_{n}=\lim _{n \rightarrow \infty} \frac{H_{n}}{n}
$$

called the entropy of information source (cryptographic system).

If $\alpha$ is a $k$-th order Markov sequence, $h_{n}=h_{S h}$ for all $n \geq k$. A Markov sequence corresponds to a deterministic process, in which the next state depends on the previous $k$ states, that is

$$
\operatorname{Pr}\left(s_{i} \mid s_{i-1}, s_{i-2}, \ldots\right)=\operatorname{Pr}\left(s_{i} \mid s_{i-1}, s_{i-2}, \ldots, s_{i-k}\right), \quad s_{i} \in \alpha .
$$

Examples of Markov process can be found in most cryptographic systems such as PRNG's and block ciphers.

\subsubsection{Entropy-Complexity Relationship}

Intuitively, complexity and the entropy are related as 'cause and effect': the more complex the internal organization of a system, the more unpredictable its behavior is and the higher the entropy is. The complexity is the size of the 'internal program' that generates a state sequence (string), whereas the entropy is computed from the probability distribution of this sequence. Formally, the following result is applied to stationary ergodic sources [28]:

$$
\lim _{n \rightarrow \infty} \frac{\left\langle K_{n}\right\rangle}{H_{n}}=\frac{1}{\ln 2}
$$

where $\left\langle K_{n}\right\rangle=\sum_{\alpha_{n} \in\{0,1\}^{n}} \operatorname{Pr}\left(\alpha_{n}\right) K\left(\alpha_{n}\right)$. Hence, the average complexity $\left\langle K_{n}\right\rangle$ is asymptotically proportional (with the coefficient $\ln 2$ ) to the entropy as $n$ increases. 


\subsection{Entropy and Complexity of Chaotic Systems}

\subsubsection{Partitioning and Symbolic Dynamics}

Consider a chaotic system $\mathcal{S}=\langle X, f\rangle$ with an $f$-invariant measure $\mu$ (see Section 2.2). Any set of $m$ disjoint regions which covers the state space $X$ is a partition denoted by

$$
\beta=\left\{X_{1}, X_{2}, \ldots, X_{m}\right\}: \quad \bigcup_{i=1}^{i=m} X_{i}=X, \quad X_{i} \cap X_{j}=\emptyset, \forall i \neq j
$$

A unique symbol $s_{i} \in \mathcal{A}$ is assigned to every region $X_{i}$. The process of partitioning the state space, assigning symbols to every region from the partition, and the resulting macroscopic dynamics are called symbolic dynamics [55]. A function $\sigma$ can define partitions and their symbolic names:

$$
\sigma(x)=\left\{s_{i} \in \mathcal{A} \mid x \in X_{i}\right\}
$$

A trajectory $\phi\left(x_{0}\right)$ passing across the subsets $X_{i}$ produces a symbolic trajectory $\alpha\left(x_{0}\right)$.

\subsubsection{Kolmogorov-Sinai Entropy}

The Lyapunov exponents (Section 2.2.4) measure how fast we loose the capability to predict the behavior of a chaotic system in time. The disadvantage is that this measure does not consider the resolution under which the system is observed, unlike the Kolmogorov-Sinai entropy [16, 9, 28].

Let the partition $\beta=\left\{X_{1}, X_{2}, \ldots, X_{m}\right\}$ be the observer's resolution. Looking at the system state $x$, the observer can only determine the fact that $x \in X_{i}$ and reconstruct the symbolic trajectory $\alpha_{n}=\left\{s_{m_{1}}, s_{m_{2}}, \ldots, s_{m_{n}}\right\}$ corresponding to the regions visited. The entropy of a trajectory $\alpha_{n}$ with the respect to partition $\beta$ is given by

$$
H_{n}^{\beta}=-\sum_{\alpha_{n}} \operatorname{Pr}\left(\alpha_{n}\right) \log _{|\mathcal{A}|} \operatorname{Pr}\left(\alpha_{n}\right)
$$

where $\operatorname{Pr}\left(\alpha_{n}\right)$ is the probability of occurrence of the substring $\alpha_{n}$. The conditional entropy of the $(n+1)$-th symbol provided the previous $n$ symbols are known is 
defined as

$$
h_{n}^{\beta}=h_{n+1 \mid n}^{\beta}= \begin{cases}H_{n+1}^{\beta}-H_{n}^{\beta}, & n \geq 1 \\ H_{1}^{\beta}, & n=1\end{cases}
$$

The entropy for a partition $\beta$ is given by

$$
h^{\beta}=\lim _{n \rightarrow \infty} h_{n}^{\beta}=\lim _{n \rightarrow \infty} \frac{1}{n} H_{n}^{\beta} .
$$

The Kolmogorov-Sinai entropy of a chaotic system is the supremum over all possible partitions

$$
h_{K S}=\sup _{\beta} h^{\beta} .
$$

The KS entropy equals zero for regular systems, is finite and positive for a deterministic chaos and infinite for a random process. It is related to the Lyapunov exponents by $h_{K S}=\sum_{1 \leq d \leq D} \lambda_{d}$ and proportional to the time horizon $T$ on which the system is predictable.

\subsubsection{Complexity of the Trajectory}

The complexity of the trajectory of a point $x_{0}$ with respect to a finite opening coverings $\beta$ is defined as

$$
\mathcal{C}^{\beta}\left(x_{0}\right)=\limsup _{n \rightarrow \infty} \frac{1}{n} \min _{\alpha_{n} \in[\psi(x)]^{n}} K\left(\alpha_{n}\right),
$$

where $[\psi(x)]^{n}=\left\{\alpha_{n} \mid f^{j}\left(x_{0}\right) \in X_{j}\right\}$ and $K\left(\alpha_{n}\right)$ is the algorithmic complexity of $\alpha$.

The complexity of the trajectory of a point $x_{0}$ is

$$
\mathcal{C}\left(x_{0}\right)=\sup _{\beta} \mathcal{C}^{\beta}\left(x_{0}\right)
$$

Definition 6 (algorithmically random trajectory, [30, 101]) The trajectory of a point $x_{0}$ is called algorithmically random if its complexity is positive, that is $c\left(x_{0}\right)>$ 0 .

The Brudno and White theorem defines the relationship between the KS entropy and complexity: 
Theorem 1 (complexity of the trajectory, $[30,101])$ The symbolic trajectories of almost all $x \in X$ (with respect to the invariant measure $\mu$ ) are algorithmically random and their complexity is given by

$$
c(x)=\frac{h_{K S}}{\ln 2}
$$

Though it is practically impossible to quantify the algorithmic complexity of a string, most strings over a finite alphabet produced by a chaotic system are algorithmically random.

\subsection{Pseudo-Randomness}

\subsubsection{Probabilistic Ensembles}

Let $\operatorname{Pr}_{i}(\alpha)$ be a probability distribution function of strings $\{0,1\}^{l(i)}$, where $l(i)$ is a positive polynomial. We write $\Pi=\left\{\operatorname{Pr}_{i}\right\}_{i \in I}$ for an ensemble of distributions indexed by $I \subset \mathbb{N}$. The ensemble of the uniform distributions $\Pi_{0}=\left\{\operatorname{Pr}_{0, i}\right\}_{i \in \mathbb{N}}$ for all $i \in \mathbb{N}$ and $\alpha, \beta \in\{0,1\}^{i}$ satisfies $\operatorname{Pr}_{0, i}(\alpha)=\operatorname{Pr}_{0, i}(\beta)$.

To measure the 'degree of randomness' of a string its probability ensemble should be compared with that of the uniform distributions. Having limited resources, computers can process only a subset of distributions. Thus, we introduce the concept of polynomial indistinguishability. Roughly speaking, two probabilistic ensembles are polynomially indistinguishable if they assign 'about the same' mass to the same subsets of strings, efficiently recognized by a Turing machine:

Definition 7 (polynomial indistinguishability, $[105,50,65]$ ) Let $\Pi_{1}=\left\{\operatorname{Pr}_{1, i}\right\}_{i \in I}$ and $\Pi_{2}=\left\{\operatorname{Pr}_{2, i}\right\}_{i \in I}$ be two probability ensembles each indexed by $I$. Let $T$ be a probabilistic polynomial-time Turing machine called a test. The test gets two inputs: an index $i$ and a string $\alpha$. Let $\operatorname{Pr}_{1}^{T}(i)$ be the probability that, on input index $i$ and a string $\alpha$ chosen according to the distribution $\operatorname{Pr}_{1, i}$, the test $T$ outputs 1 . Similarly, $\operatorname{Pr}_{2}^{T}(i)$ denotes the probability that, on input index $i$ and a string $\alpha$ chosen according to the distribution $\operatorname{Pr}_{2, i}$, the test $T$ outputs 1 . We say that $\Pi_{1}$ and $\Pi_{2}$ are indistinguishable with polynomial $p(i)$ if for all probabilistic polynomial-time tests $T$ and sufficiently large $i \in \mathbb{N}$

$$
\left|\operatorname{Pr}_{1}^{T}(i)-\operatorname{Pr}_{2}^{T}(i)\right|<\frac{1}{p(i)}
$$


Definition 8 (pseudo-random probability ensemble, [105, 50, 65]) The probability ensemble $\Pi=\left\{\operatorname{Pr}_{i}\right\}_{i \in I}$ is said to be pseudo-random if for any positive polynomial $p(i)$, the ensemble $\Pi$ is indistinguishable with $p(i)$ from the uniform ensemble $\Pi_{0}=$ $\left\{\operatorname{Pr}_{0, i}\right\}_{i \in I}$.

Definition 9 (unpredictable probability ensemble, $[105,50,65]$ ) Let $\Pi=\left\{\operatorname{Pr}_{1, i}\right\}_{i \in I}$ be a probabilistic ensemble indexed by $I$. Let $T$ be a probabilistic ensemble polynomialtime Turing machine that on input (index $i$ and a string $\alpha$ ), outputs a single bit, called the guess. Let bit $(\alpha, r)$ denote the $r$-th bit of the sequence $\alpha$, and pref $(\alpha, r)$ denote the prefix of $r$ bits of the string $\alpha$, (i.e. pref $(\alpha, r)=b i t(\alpha, 1) \operatorname{bit}(\alpha, 2) \ldots \operatorname{bit}(\alpha, r)$ ). We say that the machine $T$ predicts the next bit of $\Pi$, if for some polynomial $p(i)$ and infinitely many $i$ 's,

$$
\operatorname{Pr}(M(i, p r e f(\alpha, r))=\operatorname{bit}(\alpha, r+1)) \geq \frac{1}{2}+\frac{1}{p(i)},
$$

where the probability space is that of the string $\alpha$ chosen according to $\operatorname{Pr}_{1, i}$, and the integer $r$ chosen at random with uniform distribution in $\{0,1, \ldots, l(\alpha)-1\}$. We say that $\Pi$ is unpredictable if there exists no probabilistic polynomial time machine $T$ which predicts the next bit of $\Pi$.

Theorem $2[27,50,65]$ The probability ensemble $\Pi$ is pseudo-random if and only if $\Pi$ is unpredictable.

\subsubsection{One-Way Function}

One-way functions are functions that are easy to evaluate $(\beta=f(\alpha))$, but hard (on the average) to invert $\left(\alpha=f^{-1}(\beta)\right)$. The computational gap between forward and inverse evaluation quantifies the efficiency of the one-way transformation. This is related to the so-called inverse problems including inverse scattering theory for example, for which exact solutions are rare in practice, the problems ill-posed in most cases.

It is widely believed, but not proven that one-way functions exists. However, one-way functions lie at the heart of modern cryptography, in particular, they are used in public-key schemes. 
A function is called length-preserving (denoted by 1:1) if the binary length of its argument $\alpha$ equals the length of the result $\beta$. An important application of the one-way $1: 1$ function is the PRNG.

If a one-way function is not length-preserving [length $(\alpha)>\operatorname{length}(\beta)]$, it is called a hash-function. Hash functions are used, for example, in digital signature schemes.

A formal definition of one-way function is given in terms of complexity theory:

Definition 10 (one-way function $[27,50,65]$ ) A function $f:\{0,1\}^{*} \rightarrow\{0,1\}^{*}$ is called one way if it satisfies the following

1. There is a deterministic polynomial-time Turing machine that on input $\alpha$ returns $f(\alpha)$

2. For any probabilistic polynomial-time Turing machine $M$, any positive polynomial $p(n)$ and sufficiently large $n$

$$
\operatorname{Pr}\left(M\left(f(\alpha), 1^{n}\right) \in f^{-1}(\alpha)\right)<\frac{1}{p(n)},
$$

where the probability is taken over all possible choices of $\alpha \in\{0,1\}^{n}$ and the internal tosses of $M$ conform to a uniform probability distribution. The role of $1^{n}$ is to allow machine $M$ to run in a time polynomial over the length of the pre-image it is supposed to find.

A stronger notion of unpredictability is that of a hard-core predicate. A polynomialtime computable predicate $b$ is called a hard-core of a function $f$ if all algorithms, given $f(\alpha)$, can guess $b(\alpha)$ only with a probable success which is negligibly better than half.

Definition 11 (hard-core predicate, $[27,50,65])$ Let $f:\{0,1\}^{*} \rightarrow\{0,1\}^{*}$ and $f:\{0,1\}^{*} \rightarrow\{0,1\}$. The predicate $b$ is said to be hard-core of the function $f$, if

1. There is a deterministic polynomial-time Turing machine that on input $\alpha$ returns $b(\alpha)$;

2. There is no probabilistic polynomial-time Turing machine $M$ such that for any positive polynomial $p(n)$ and sufficiently large $n$

$$
\left.\operatorname{Pr}\left(M\left(f(\alpha), 1^{n}\right)=b(\alpha)\right)\right)<\frac{1}{2}+\frac{1}{p(n)},
$$


where the probability is taken over all possible choices of $\alpha \in\{0,1\}^{n}$ and the internal tosses of $M$ conform to a uniform probability distribution.

Theorem 3 (existence of a one-way function with a hard-core predicate, [105, 72, 50]) If there exists a one-way function, then there exists a one-way function with a hard-core predicate.

\subsubsection{Pseudo-Random Generators}

The role of pseudo-random (number) generators (PRNG) in cryptography has already been discussed in Section 2.1.4. Roughly speaking, a PRNG is an efficient (deterministic) algorithm that on input a short seed, outputs a (typically much) longer sequence that is computationally indistinguishable from a uniformly chosen string.

Definition 12 (pseudo-random generator, [27, 50]) Let $l: \mathbb{N} \rightarrow \mathbb{N}$ satisfy $l(n)>$ $n$ for all $n \in \mathbb{N}$. A pseudo-random generator, with a stretch function $l(n)$, is a deterministic polynomial time algorithm $G$ satisfying the following:

1. For every $\alpha \in\{0,1\}^{*}$ it holds that $|G(\alpha)|=l(|\alpha|)$

2. The probabilistic ensembles $\Pi=G\left(\mathrm{Pr}_{0}^{n}\right)$ and $\Pi_{0}^{p(n)}$ are computationally undistinguishable.

Theorem 4 (construction of a pseudo-random generator, $[105,50]$ ) Let $f$ be a oneway $1: 1$ function, and $b$ be a hard-core predicate of $f$. Then

$$
G(\alpha)=b(\alpha) b(f(\alpha)) \ldots b\left(f^{l(|\alpha|)-1}(\alpha)\right)
$$

is a pseudo-random generator with a stretch function $l$.

Consequently, a pseudo-random generator can be constructed from any one-way length-preserving function (rather then merely one-way permutations). On the other hand, the existence of a one-way function is a necessary condition to the existence of the pseudo-random generator, that is

Theorem 5 (existence of pseudo-random generators, $[60,56,50]$ ) Pseudo-random generators exists if and only if one-way functions exists. 

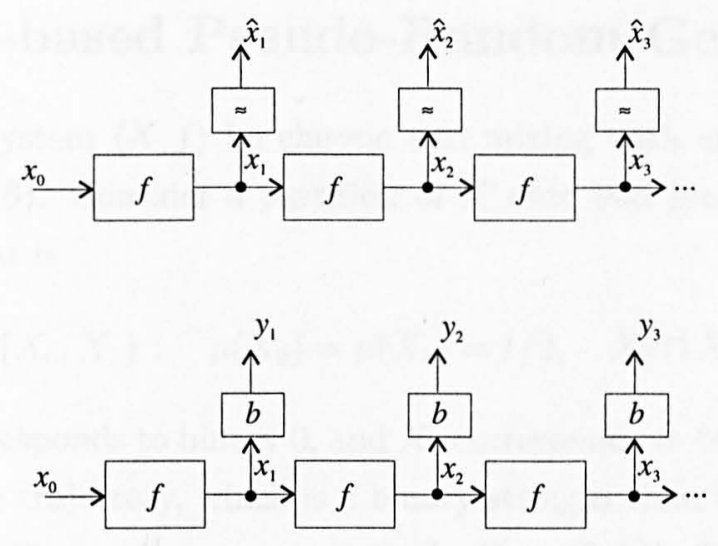

Figure 3.3: A synergy between a chaotic system (top: $\approx$ is a rounding function, $\hat{x_{n}}$ is the output) and a PRNG (bottom: $b$ is a hard-core predicate, $y_{n}$ is the output).

Assuming the existence of one-way 1:1 functions, there can exist probability distributions, which are not uniform and are not even statistically close to a uniform distribution, that, nevertheless, are computationally indistinguishable from a uniform distribution [65].

The definition of a pseudo-random generator given above cannot be applied directly since there is no practical way to prove or check rigourously indistinguishability.

Practical cryptography is based on passing known statistical tests (see, for example, $[85,88,74,77])$, which ensure the pseudo-random property of a generator. Moreover, it is considered that pseudo-random sequences can be used instead of truly random sequences in most cryptographic applications. Chapter 6 describes a basic approach to these statistical tests and evaluates a number of pseudo-chaotic systems.

A parallel between pseudo-random generators and chaotic systems is drawn in Figure 3.3. Though the structure looks similar, there is a fundamental difference: the iterated function of a chaotic system is not required to be one-way. Chaos theory pays no attention to the algorithmic complexity of $f$ and $f^{-1}$, which is one of the main problems when we apply a chaotic system to cryptography. 


\subsection{Chaos-based Pseudo-Random Generators}

Let the dynamic system $\langle X, f\rangle$ be chaotic and mixing with an $f$-invariant measure $\mu$ (Section 2.2.6). Consider a partition of $X$ onto two $\mu$-equiprobable subsets (Section 3.4.1), that is

$$
\beta=\left\{X_{0}, X_{1}\right\}: \quad \mu\left(X_{0}\right)=\mu\left(X_{1}\right)=1 / 2, \quad X_{0} \cap X_{1}=\emptyset
$$

The subset $X_{0}$ corresponds to binary 0 , and $X_{1}$ corresponds to binary 1 . The system outputs a symbolic trajectory, which is a binary string $\alpha$ from the initial condition (the seed) $x_{0} \in X$. Denote the generator as $G: X \rightarrow\{0,1\}^{*}$. Then

$$
G(x)=\alpha=\left\{s_{i}\right\}_{i=1,2, \ldots}, \quad x \in X, \quad s_{i} \in\{0,1\} .
$$

Szczepankski and Kotulski $[95,68]$ showed that $G$ can produce asymptotically random sequences. The next theorem affirms that if we take two different seeds, then with probability one, we obtain two different sequences:

Theorem 6 (the uniqueness of the trajectory, [95]) For all $x \in X$,

$$
\mu\left(G^{-1}(\alpha)\right)=0
$$

Ergodically, we expect that the number of zeros in the generated sequence is equal to the expected number of ones. In particular, the Birkhoff-Khinchin Ergodic Theorem [9] can be written as

$$
\begin{aligned}
& \lim _{n \rightarrow \infty} \frac{1}{n} \sum_{i=0}^{n-1} \chi_{X_{0}}\left(f^{i}(x)\right)=\int_{X_{0}} \chi_{x_{0}} d \mu=\mu\left(X_{0}\right), \\
& \lim _{n \rightarrow \infty} \frac{1}{n} \sum_{i=0}^{n-1} \chi_{X_{1}}\left(f^{i}(x)\right)=\int_{X_{1}} \chi_{X_{1}} d \mu=\mu\left(X_{1}\right),
\end{aligned}
$$

where $\chi_{x_{0}}, \chi_{X_{1}}$ are indicator functions of $X_{0}$ and $X_{1}$ respectively. Since $\mu\left(X_{0}\right)=$ $\mu\left(X_{1}\right)=1 / 2$, the average number of zeros and ones tends to $n / 2$.

Theorem 7 (satisfying of the monobit test, [95]) For all $x \in X$, the number of zeros and ones in $\alpha$ are approximately the same. 


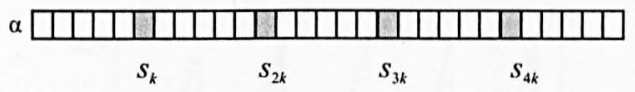

Figure 3.4: A rarefied sample for $k=6$.

The mixing property ensures the asymptotic independence of bits:

Theorem 8 (asymptotic independence, [95]) For all $x \in X$ and $n=1,2, \ldots$, output bits $s_{(n-1) k}$ and $s_{n k}$ (considered as random variables) are asymptotically independent as $k$ increases, that is

$$
\lim _{k \rightarrow \infty} \mu\left(f^{-n k-k}\left(X_{0}\right) \cap f^{-n k}\left(X_{1}\right)\right)=\mu\left(f^{-n k}\left(X_{0}\right)\right) \cdot \mu\left(f^{-n k}\left(X_{1}\right)\right)
$$

and

$$
\lim _{k \rightarrow \infty} \mu\left(f^{-n k-k}\left(X_{1}\right) \cap f^{-n k}\left(X_{0}\right)\right)=\mu\left(f^{-n k}\left(X_{1}\right)\right) \cdot \mu\left(f^{-n k}\left(X_{0}\right)\right) .
$$

Hence, as $k$ increases, the autocorrelation function of the string $s_{k}, s_{2 k}, \ldots, s_{n k}, \ldots$ (Figure 3.4) tends to a delta function. Practically, the function composition $f^{k}$ increases nonlinearity of the relationship between $x_{n k}$ and $x_{(n-1) k}$.

So far, by dropping intermediate states from a cryptographic sequence, we improve its pseudo-random appearance. Also, asymptotical independence forms the basis of multi-round block encryption schemes and ensures that the ciphertext is statistically independent of the plaintext.

\section{An Example of Chaos based PRNG}

Consider the sawteeth map (Figure 3.5)

$$
x_{n+1}=r x_{n} \bmod q,
$$

where $x_{0} \in[0, q], r=p / q>1$, and $p$ is co-prime to $q$. The map is chaotic for all $r$ and has Lyapunov exponent $\lambda=h_{K S}=\log r>0$.

A discrete version of the sawteeth map is well known in conventional cryptography. The classical Linear Congruental Generator (LCG) is given by

$$
x_{n+1}=\left(A x_{n}+C\right) \bmod M \text {, }
$$




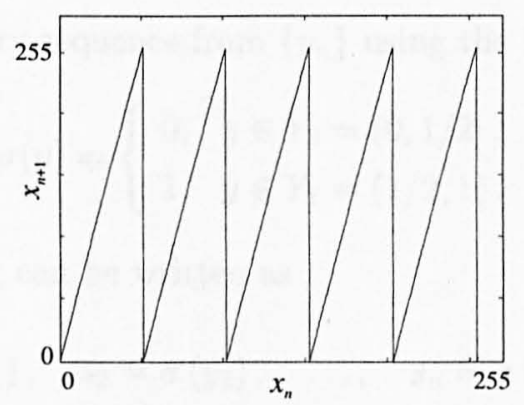

Figure 3.5: The sawteeth map for $p=1279$ and $q=255$.

where $x_{n} \in\{0,1, \ldots, M\}$ and $A, C, M$ are fixed by the designer [64]. The continuous sawteeth system (and LCG) shows the stretch and folder property of chaos: first, the the state is stretched over a large space (e.g, multiplying or raising in power); then folded $q$ times into a smaller state space (using a periodical function such as $\bmod , \sin$ etc).

However, the continuous sawteeth map, unlike the LCG, cannot tell us about its finite-state approximation, especially about the periodical orbits resulting in patterns.

Theoretically, for large $p$ and $q$, the sawteeth map is a one-way function: given $x_{n}$ one can only guess among $q$ equally distributed values what was the previous element $x_{n-1}$. In each iteration much information is lost, and the inverse sawteeth map is highly multi-valued.

By applying a periodical piecewise-linear function $g$ to each element of the chaotic sequence $\left\{x_{n}\right\}$, i.e.

$$
y_{0}=g\left(x_{0}\right), \quad y_{1}=g\left(x_{1}\right), \quad \ldots, \quad y_{n}=g\left(x_{n}\right), \quad \ldots
$$

we obtain a new chaotic sequence $\left\{y_{n}\right\}$ that is unpredictable in both ways: given $y_{n}$, one can only guess among equally distributed values what was the previous element $y_{n-1}$ and what will be the next element $y_{n+1}$. Periodicity provides a strong fold effect ( $g^{-1}$ becomes highly multi-valued), while piecewise-linearity ensures that the uniform distribution of $\left\{x_{n}\right\}$ is passed to $\left\{y_{n}\right\}$. For example, the function $g$ can be another (or the same) sawteeth map. 
We can obtain a binary sequence from $\left\{y_{n}\right\}$ using the $1 / 2$ threshold:

$$
\sigma(y)= \begin{cases}0, & y \in Y_{0}=(0,1 / 2] \\ 1, & y \in Y_{1}=(1 / 2,1) .\end{cases}
$$

Finally, the output string can be written as

$$
s_{1}=\sigma\left(y_{1}\right), \quad s_{2}=\sigma\left(y_{2}\right), \quad \ldots, \quad s_{n}=\sigma\left(y_{n}\right) \quad \ldots
$$

Thus, by defining a chaotic function $f$ with control parameters, an additional transformation $g$ and a partition function $\sigma$, a pseudo-random generator can be constructed. The combined effect of $g$ and $\sigma$ is that of a hard-core predicate.

However, the one-step unpredictability of $\left\{y_{n}\right\}$ does not guarantee that the sequence will be unpredictable when an adversary has access to a sufficiently long sequence $\left\{s_{n}\right\}$. In other words, the vast number of samples can theoretically lead to the predictability of $\left\{s_{n}\right\}$. 


\section{Chapter 4}

\section{Pseudo-Chaotic \\ Cryptographic Systems}

This chapter studies cryptographic systems based on finite-state approximations of chaos (i.e. pseudo-chaos). Two approaches are considered to store the system state on a computer: (i) the floating-point format of real numbers and (ii) 'plain' binary strings or $m$-dimensional cubes.

\subsection{Chaos and Pseudo-Chaos}

In the previous chapter we have constructed a pseudo-random generator from a chaotic system. This generator produces infinite algorithmically random strings, furthermore, rarefied samples from these strings are asymptotically uncorrelated as the distance $k$ increases. However, this result relates to truly chaotic systems with infinite number of states, whereas digital cryptography is based on finite-state computers.

The notion of pseudo-chaos introduced in $[34,26]$ denotes a computer approximation of chaos. The fundamental differences between mathematical chaos and pseudo-chaos are the following:

- The state variable has a finite length, i.e. stores the state with a finite precision. The system has a finite number of states $N$. 


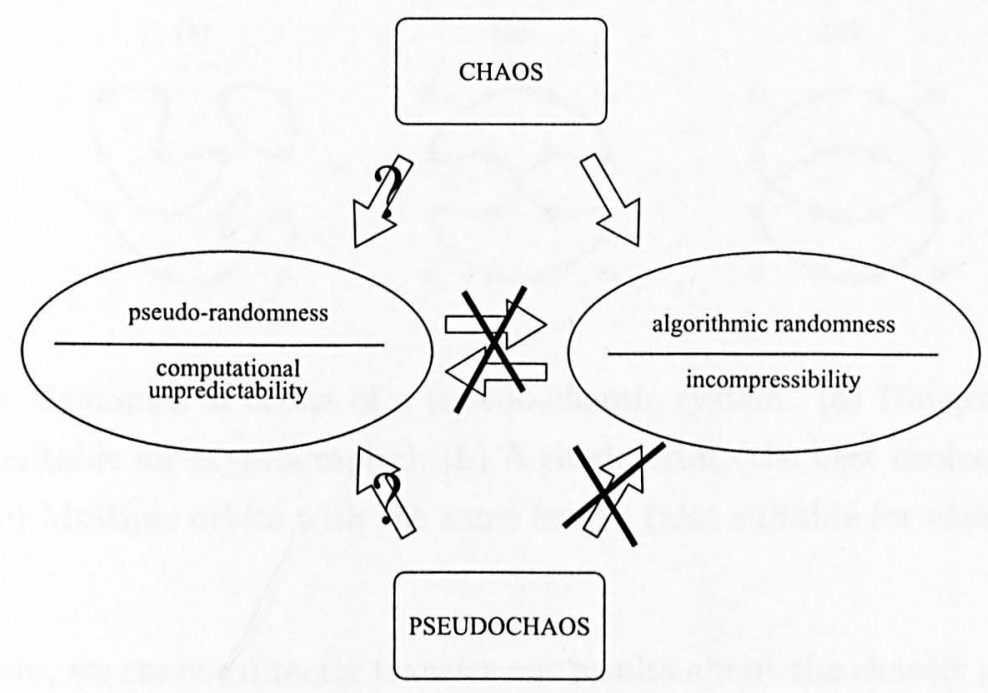

Figure 4.1: Properties of chaotic and pseudo-chaotic systems.

- The iterated function is evaluated with approximate methods, the result is rounded (or truncated) to a finite precision.

- The system is observed during a finite period of time.

The central problem is that rounding is applied in each iteration and the error accumulation causes the original and the approximated processes to diverge very fast. As was first discovered by Lorenz ${ }^{1}$, “. . a small error in the former will produce an enormous error in the future. Prediction becomes impossible ...". Pseudo-chaos is a poor approximation of chaos since the approximated model does not converge with the original model, and, formally, exhibits non-chaotic properties:

- All trajectories are eventually periodic, i.e. contain patterns. A cycle appears as soon as two states are rounded to the same approximated value.

- Consequently, the Lyapunov exponent $\lambda$ (Section 2.2.4) and the KS entropy $h_{K S}$ (Section 3.4.2) trivially equal 0.

\footnotetext{
${ }^{1}$ Lorenz, Edward, is an American meteorologist, who discovered a stable chaotic attractor in the 1960 s.
} 
(a)

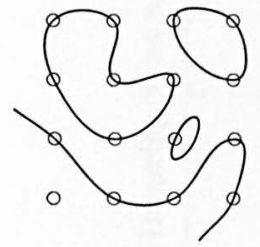

(b)

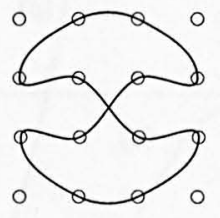

(c)

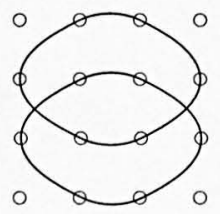

Figure 4.2: Examples of orbits of a pseudo-chaotic system. (a) Dangerously short orbits (unsuitable for cryptography); (b) A single orbit (the best choice for cryptography); (c) Multiple orbits with the same length (also suitable for encryption).

Therefore, we cannot directly transfer our results about the chaotic generator to computer approximations (as summarized in Figure 4.1). The methods from chaos theory should be adopted for pseudo-chaotic systems and cryptographic applications.

What are the minimal, typical and maximal periods of the orbits? Such questions are important in most cryptographic systems. In general, a pseudo-chaotic system produces orbits with different lengths (sometimes called random-length orbits) as illustrated in Figure 4.2-a. Of course, such patterns constitute serious vulnerability: a system may have weak plaintexts and weak keys resulting in recognizable ciphertexts.

If a system has a stable attractor for all initial conditions and parameters, and all orbits have (almost) the same length (Figure 4.2-c), there are more chances to develop a secure encryption scheme. Nevertheless, multiple orbits narrow the search space during cryptanalysis. An ideal cryptosystem has a single orbit passing through the whole state space (Figure 4.2-b).

The next step is to estimated $\lambda$ of a typical orbit for time not exceeding its period. However, the analysis of periodic orbits depends crucially on the ordering with which the orbits are considered [66]. Two orderings, both corresponding to Lebesgue measure, are considered in the literature: ordering according to the system size $N$, and ordering according to the minimal period and then lexicographically within the same period.

In the case of the sawteeth map (3.8), with $r=2$, two different orderings lead to two opposite answers [66]: ordering by system size yields logarithmic compressibility 


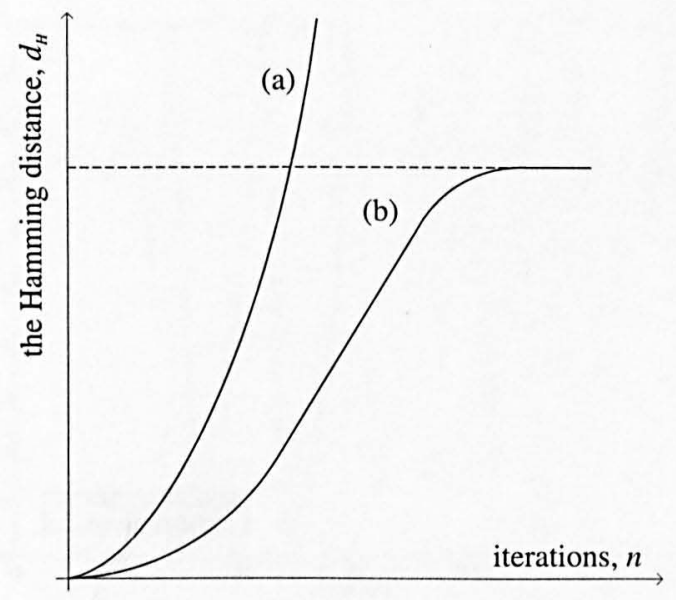

Figure 4.3: A Laypunov exponent of a chaotic (a) and pseudo-chaotic (b) system

of information and zero finite-time (or lack of randomness), while ordering by the minimal period leads to positive finite-time $\lambda$ and randomness.

A comparison between the average Lyapunov curve of a chaotic system and an analogous property of a pseudo-chaotic system is given in Figure 4.3. If the pseudochaotic system has a finite precision $\sigma$, then the exponential divergence given by

$$
e^{n \lambda}=\frac{\left|f^{n}\left(x_{0}+\varepsilon\right)-f^{n}\left(x_{0}\right)\right|}{\varepsilon}, \quad n \rightarrow \infty, \varepsilon \rightarrow 0,
$$

will be eventually limited by $\varepsilon=\sigma$. Usually the fraction (4.1) grows exponentially during the first few iterations and then increases linearly until it finally levels off at a certain finite value.

\subsection{Floating-point Approximations of Chaos}

A floating-point and fixed point arithmetic [58] are the most straightforward solution of approximating a continuous system on a finite state machine (computer). Both approaches imply that the state of a continuous system is stored in a program variable under a finite resolution. A state variable $x$ can be written as a binary fraction $b_{m} b_{m-1} \ldots b_{1}, a_{1} a_{2} \ldots a_{s}$, where $a_{i}, b_{j}$ are bits, $b_{m} b_{m-1} \ldots b_{1}$ denotes the integer part and $a_{1} a_{2} \ldots a_{s}$ is the fractional part of $x$. Under a finite resolution, 


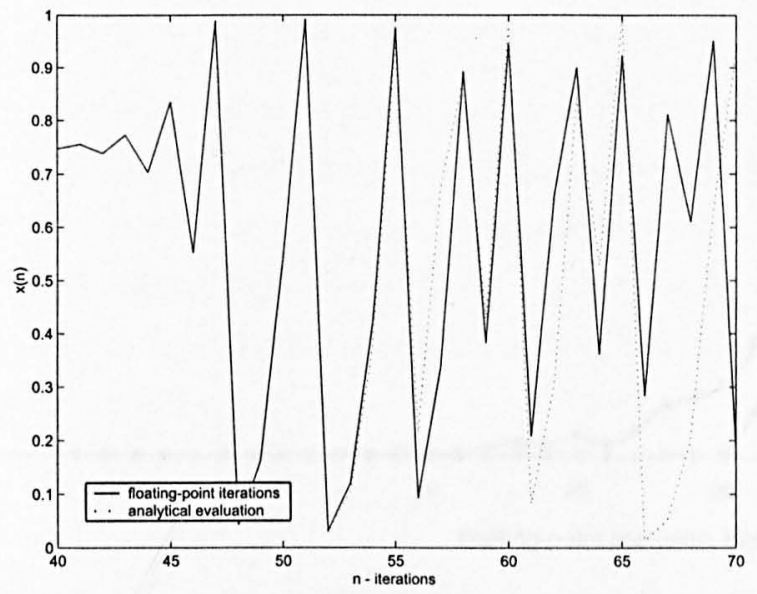

Figure 4.4: Trajectories of a continuous-state chaotic system (4.4) and its 64-bit floating-point approximation. The first curve is obtained by means of the analytical solution (4.5). The rounding off error is amplified in each iteration and the trajectories diverge exponentially.

instead of $x_{n+1}=f(x)$, we write

$$
x_{n+1}=\operatorname{round}_{k}\left(f\left(x_{n}\right)\right),
$$

where $k \leq s$ and $\operatorname{round}_{k}(x)$ is a rounding function defined as

$$
\operatorname{round}_{k}(x)=b_{m} b_{m-1} \ldots b_{1} \cdot a_{1} a_{2} \ldots a_{k-1}\left(a_{k}+a_{k+1}\right) .
$$

The iterative rounding is accumulative and results in surprisingly different behavior of pseudo-chaos compared with its continuum counterparts. Figure 4.4 shows how fast the original and approximated trajectories diverge.

For cryptographic applications, the rounding off function exposes another danger. Rounding or truncating the state (e.g. to zero values) can lead the process coming out of the chaotic attractor. After that, the system state typically directs to a certain constant value or infinity. Thus, we have to exclude some forbidden initial conditions and parameters, which yield a short orbit or patterns of behavior after a small number of iterations. Figure 4.5 is a plot of the average cycle length verses floatingpoint precision. It shows that a high precision does not guarantee a sufficiently long trajectory. 


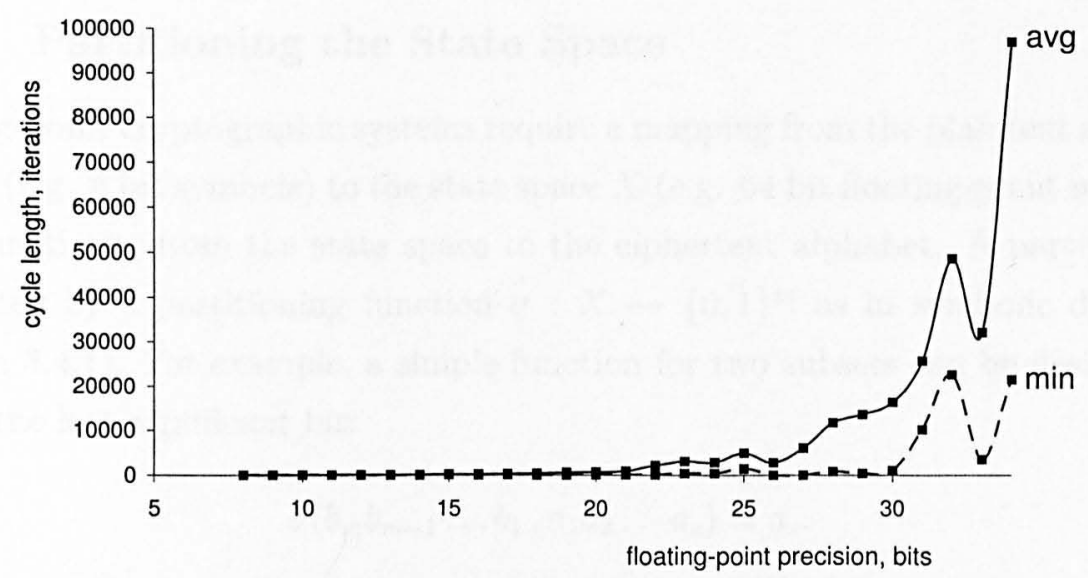

Figure 4.5: The average and the minimal cycle length of the logistic system (4.4) verses floating-point precision obtained from 10 samples of the logistic system.

Another problem is the sensitivity to floating-point processor implementations. Diversified mathematical algorithms or internal precisions in intermediate calculations can lead to the situation when the same code of an encryption software will generate different cryptographic sequences, so there will be no compatibility between software environments.

In Section 3.6 we have mentioned that a chaos-based PRNG with two different seeds produces two different sequence with probability 1 . This is true for chaotic systems with an infinite state space, where the probability $\operatorname{Pr}\left(f\left(x_{n}\right)=f\left(x_{n}^{\prime}\right)\right) \rightarrow 0$ with $x_{n} \neq x_{n}^{\prime}$ (despite of the fact that $f^{-1}$ is multi-valued). In finite-state approximations, the probability of mapping two points into one is much higher (for example, in Figure $4.7 x_{n}=0.2$ and $x_{n}=0.8$ are mapped to the same point $x_{n+1}$.). Furthermore, it can occur in each iteration, so that the significant number of trajectories will have identical end routes.

In spite of these shortcomings, a number of investigators have explored the applications of continuous chaos to digital cryptography. In the following Sections, an overview on encryption schemes based on the floating-point approximation of chaos is given. 


\subsubsection{Partitioning the State Space}

Floating-point cryptographic systems require a mapping from the plaintext alphabet $\{0,1\}^{m}$ (e.g. 8 bit symbols) to the state space $X$ (e.g. 64 bit floating-point numbers) and, sometimes, from the state space to the ciphertext alphabet. A partition can be defined by a partitioning function $\sigma: X \rightarrow\{0,1\}^{m}$ as in symbolic dynamics (Section 3.4.1). For example, a simple function for two subsets can be designed by taking the last significant bit:

$$
\sigma\left(b_{m} b_{m-1} \ldots b_{1} . a_{1} a_{2} \ldots a_{s}\right)=a_{s}
$$

If a floating-point system is a pseudo-random generator, the function $\sigma$ must be irreversible like a hard-core predicate (Section 3.5.2). This can be archived with an equiprobable mapping as suggested in Section 3.6: partitions are selected in such a way, that each symbol occurs with the same probability.

However, it is not obligatory to cover all the state space or assign symbols to all partitions. On the contrary, we can change statistical properties of the resulting symbolic trajectory by assigning symbols in a particular way. For example, Figure 4.12 shows a probability distribution of state points in the attractor of a logistic system. Choosing regions with almost the same probability mass, we obtain better statistics in the output.

The number of subsets can be increased, for example, up to 4, 8, 16 etc. In this case the generator will produce more pseudo-random bits per iteration $(m=2,3,4)$. Clearly, increasing $m$ reduces the cryptographic strength of the generator since it becomes easier to invert $\sigma$.

\subsubsection{Chebyshev Map}

In 1983 Erber et al. [41] suggested using a Chebyshev mixing polynomial to simulate a random process on digital computers. The polynomial is given by

$$
x_{n+1}=x_{n}^{2}-2, \quad x_{n} \in(-2,2) .
$$

The sequence $x_{0}, x_{1}, x_{2}, \ldots$ is chaotic, however, some particular values for $x_{n}$ must be avoided $\left(x_{n} \neq-1,0,1\right)$. With a non-integral seed $x_{0}$, it is theoretically expected 


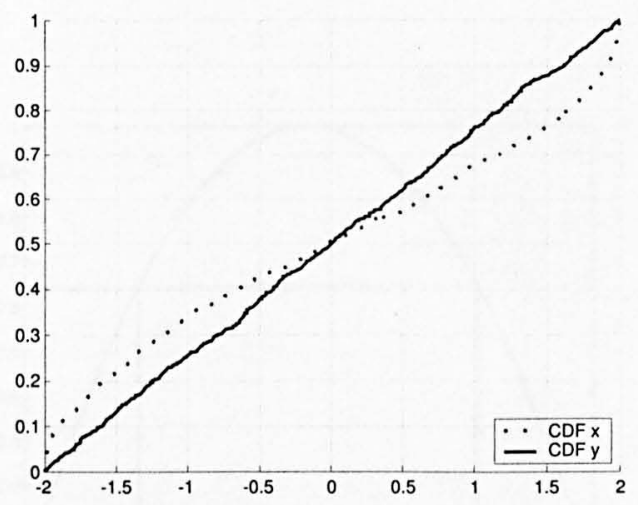

Figure 4.6: The empirical Cumulative Distribution Function of a sequence generated with the Chebyshev system before (doted line) and after (solid line) the additional transformation (4.3). The straight line looks like the uniform distribution on $(-2,2)$.

that these values will never be encountered. A system with 40-bit precision provides an average cycle of 105 steps [86].

The output $y_{n}$ is obtained after an additional nonlinear transformation

$$
y_{n}=\frac{4}{\pi} \arccos \left(\frac{x_{n}}{2}\right)-2
$$

This additional transformation attempts to improve the statistical properties of the sequence, which has a particular probability distribution (the same as for the logistic map given in Figure 4.12).

By applying a transformation with the same shape as the Cumulative Distribution Function (CDF) $\operatorname{Pr}\left(x_{n}<x\right)$ of the input sequence, one can obtain a uniform-like $\mathrm{CDF}$ of the output (Figure 4.6). However, the transformation does not affect the order of elements, so the Chebyshev sequence is highly correlated.

So far, several investigators (Hosack [59], for example) have found that the Chebyshev transformation possesses undesirable qualities which make it unsuitable for pseudo-random number generation.

\subsubsection{Logistic Map}

A similar transformation has become of the most famous chaotic maps. In 1976, Mitchell Feigenbaum studied the complex behavior of the so-called logistic map 


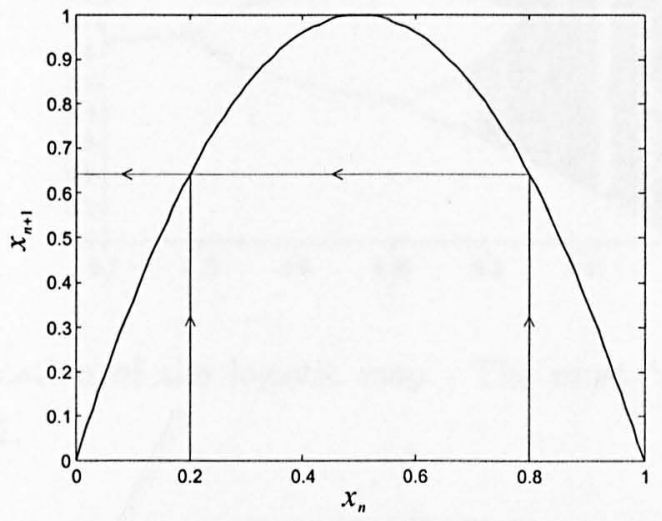

Figure 4.7: The logistic map for $r=0.99$.

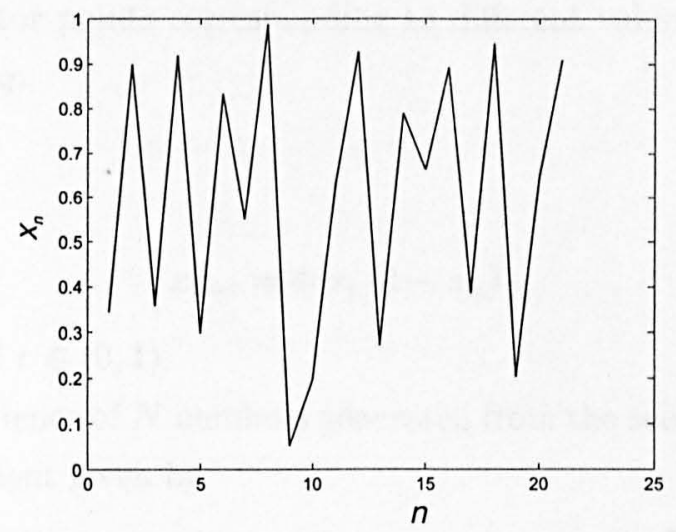

Figure 4.8: A chaotic sequence generated with the logistic map for $x_{0}=0.34$ and $r=0.99$. 


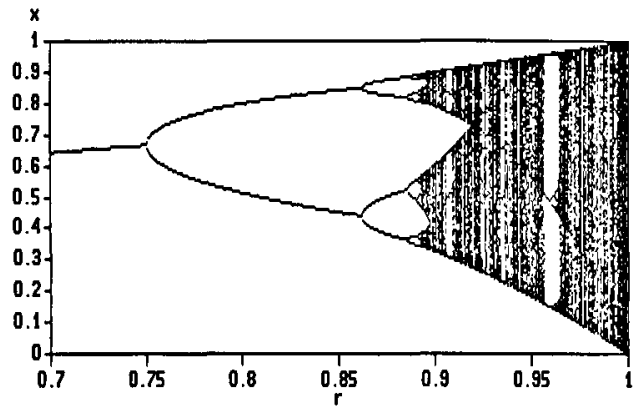

Figure 4.9: Bifurcation of the logistic map. The most 'unpredictable' behavior occurs when $r \rightarrow 1$.

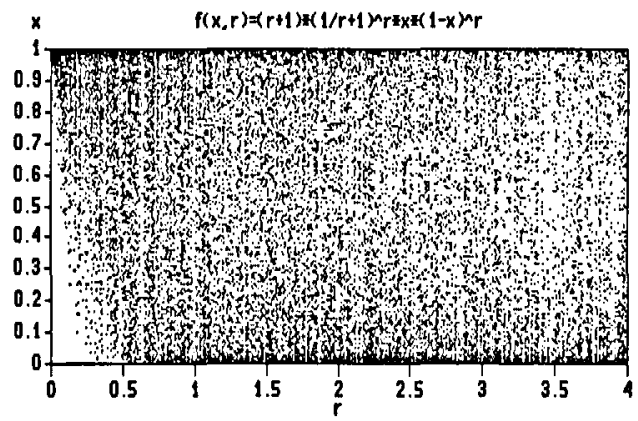

Figure 4.10: Attractor points corresponding to different values of the parameter $r$ in the Matthews map.

(Figure 4.7)

$$
x_{n+1}=4 r x_{n}\left(1-x_{n}\right)
$$

where $x \in(0,1)$ and $r \in(0,1)$.

For any long sequence of $N$ numbers generated from the seed $x_{0}$ we can calculate the Lyapunov exponent given by

$$
\lambda\left(x_{0}\right)=\frac{1}{N} \sum_{n=1}^{N} \log \left|r\left(1-2 x_{n}\right)\right| .
$$

For example, the numerical estimation for $r=0.9$ and $N=4000$ is $\lambda(0.5) \approx 0.7095$.

With certain values of the parameter $r$, the generator delivers a sequence, which appears pseudo-random (Figure 4.8). The Freigenbaum cascade (Figure 4.9) shows 


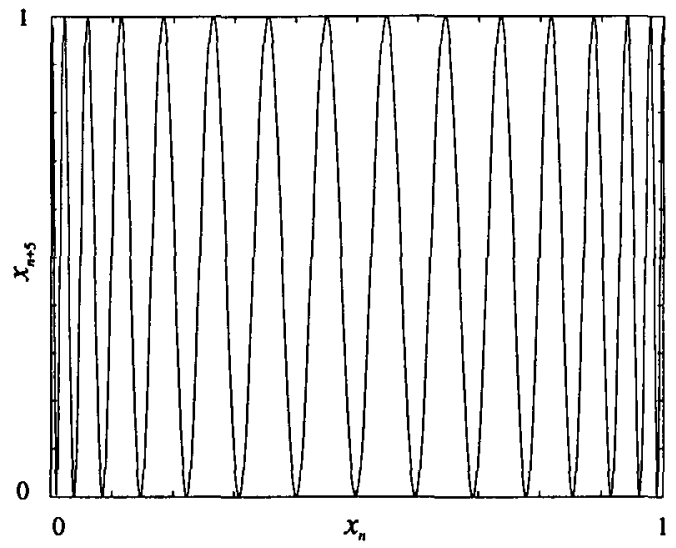

Figure 4.11: The analytical solution at $n=5$ of the logistic map for $r=1$.

the values of $x_{n}$ on the attractor for each value of the parameter $r$. As $r$ increases, the number of points in the attractor increases from 1 to $2,4,8$ and infinity. In this area $(r \rightarrow 1)$ it was considered difficult to estimate the final state of the system (without performing $n$ iterations) given initial conditions $x_{0}$, or vice-versa - to recover $x_{0}$ (which can be a key or a plaintext) from $x_{n}$. This complexity was regarded as a fundamental advantage in using continuous chaos for cryptography.

For the boundary value of the control parameter $r=1$ the analytical solution $[62,51]$ is:

$$
x_{n}=\sin ^{2}\left(2^{n} \arcsin \sqrt{x_{0}}\right) .
$$

When $n=1$ we have the initial equation (4.4).

Hence, the state $x_{n}$ can be computed directly from $x_{0}$ (without performing $n$ iterations). Figure 4.11 gives the solution for $x_{5}=f^{5}\left(x_{0}\right)$. The number of spikes increases with $n$, illustrating the sensitivity to the initial conditions. A discussion on the analytical solution is presented in Section 4.2.8.

Bianco et al. [25] used the logistic map (4.4) to generate a sequence of floating point numbers, which is then converted into a binary sequence. The binary sequence is XOR-ed with the plaintext, as in the one-time pad cipher (see Section 2.1.3). The parameter $r$ together with the initial condition $x_{0}$ form a secret key. The conversion from floating point numbers to binary values is done by choosing two disjoint interval ranges representing 0 and 1 . The ranges are selected in such a way, 


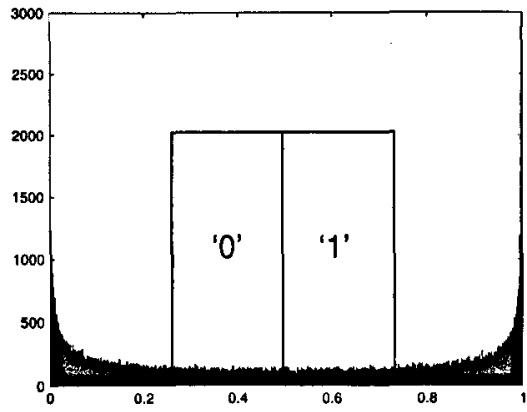

Figure 4.12: The Probability Density Function of a state sequence produced by the logistic system with an incomplete partition.

that the probabilities of occurrence of 0 and 1 are equal (as illustrated in Figure 4.12). Note, the equiprobable mapping does not ensure the uniform distribution. Though the numbers of zeros and ones are equal, the order is not random.

It has been pointed out by Wheeler [99] and Jackson [61] that computer implementations of chaotic systems yield surprisingly different behavior, i.e. it produces very short cycles and trivial patterns (a numeric example in this thesis is given in Figure 4.5).

Matthews [76] generalizes the logistic map with cryptographic constraints and develops a new map to generate a sequence of pseudo-random numbers

$$
x_{n+1}=(r+1)\left(\frac{1}{r}+1\right)^{r} \cdot x_{n}\left(1-x_{n}\right)^{r}, r \in(1,4) .
$$

The Matthews system exhibits chaotic behavior for parameter values within an extended range (Figure 4.10) extending the key space. However, no robust cryptographic system was created due to the general floating-point problems, mentioned above.

\subsubsection{Tent Map}

The one-dimension tent map is defined as

$$
x_{n+1}=\left\{\begin{array}{ll}
a \cdot x_{n}, & 0 \leq x_{n} \leq a \\
\frac{1-x_{n}}{1-a}, & a<x_{n} \leq 1
\end{array},\right.
$$




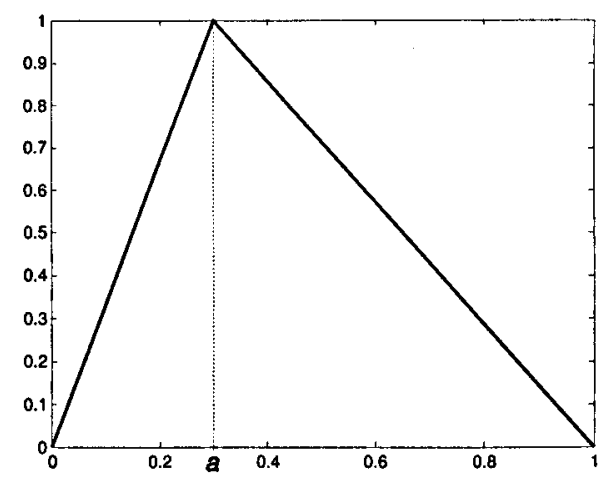

Figure 4.13: The tent map.

where the parameter $a$ determines the top of the tent (Figure 4.13).

The Lyapunov exponent depends on the parameter $a$ and is given by $\lambda(a)=$ $-a \ln (a)-(1-a) \ln (1-a)$ for almost all $x_{0} \in(0,1)[71]$.

Numerically, $\max _{a \in(0,1)} \lambda(a) \approx 0.693$ at $a=0.5$.

The tent map has an analytical solution (again, only for $a=0.5$ ) given by

$$
x_{n}=\frac{1}{\pi} \arccos \left(\cos 2^{n} \pi x_{0}\right) \text {. }
$$

Habutsu et al. [53] suggested a chaos-based block encryption system (Figure 2.3 ) in which the inverse of the one-dimensional tent map (4.6) is applied $N=75$ times to an initial condition representing the plaintext. The 64-bit plaintext is encrypted into 147-bit ciphertext (Figure 2.3). A combination of a floating-point number $a \in[0.4,0.6]$ and a binary sequence $r=\left\{r_{n}\right\}_{n \in[1, N]}$ is the secret key. The map can be written as

$$
x_{n+1}=\left\{\begin{array}{ll}
a \cdot x_{n}, & r_{n}=0 \\
(a-1) x_{n}+1, & r_{n}=1
\end{array}, \quad n \in[1, N] .\right.
$$

where $r_{i} \in\left\{r_{i}\right\}_{1}^{N}$ and $a \in[0.4,0.6]$. For $N$ inverse iterations there are $2^{N}$ possible ciphertexts which encode the same plaintext.

Biham [24] pointed out that the cryptosystem could be easily broken using a chosen ciphertext type of attack; the complexity of a known plain-text type of attack is $2^{38}$. 


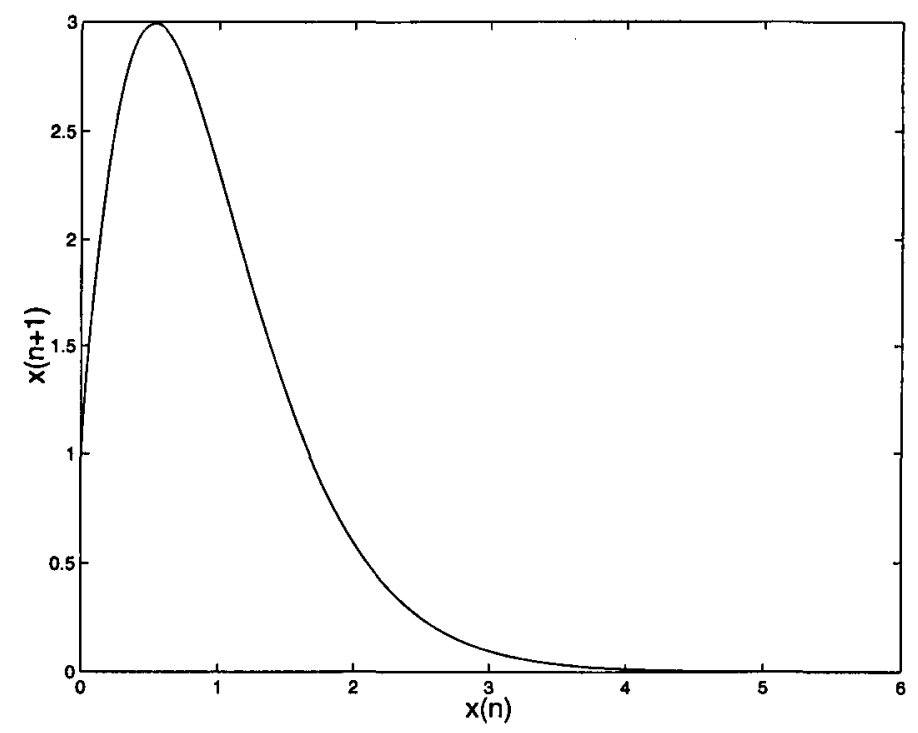

Figure 4.14: The Gallagher map.

\subsubsection{Other Chaotic Maps}

Gallagher et el. [49] developed a chaotic stream cipher, based on the transformation (Figure 4.14)

$$
f(x)=\left(a+\frac{1}{x}\right)^{\frac{x}{a}} \quad x \in(0,10), \quad a \in[0.29,0.40] .
$$

Both the initial condition $x_{0}$ and the parameter $a$ represent the key. After $n_{0}=$ 200 iterations, the system encrypts the plaintext byte $p_{1}$ into the ciphertext float $c_{1}=f^{n_{0}+n_{1}}\left(x_{0}\right)$, i.e. the chaotic map is applied $p_{1} \in[0,255]$ times. Subsequent plaintexts are encrypted using the same trajectory (Figure 2.4-c):

$$
c_{i}=f^{\left(\sum_{i=0}^{p_{i}} n_{i}\right)}\left(x_{0}\right) .
$$

Clearly, disadvantages of such an encryption scheme are: (i) the data expansion (the floating-point representation of $c_{i}$ is considerably larger that the source byte $p_{i}$ ) and (ii) unstable cycles, incident to floating-point chaos generators.

Kotulski [69] proposes a two dimensional map matching the reflection law of a geometric square and defines conditions under which the system is chaotic and mixing. 
There are, in principle, an unlimited number of iterated functions available to generate cryptographic sequences and the nonlinear transformation can be more complex, for example,

$$
\begin{gathered}
f(x)=r x\left(1-\tan \left(\frac{1}{2} x\right)\right), \\
f(x)=r x(1-\log (1+x)) .
\end{gathered}
$$

Though each system has a particular state distribution in the phase space, qualitatively, its behavior is similar to a basic chaotic system such a logistic map.

To increase unpredictability (i.e. the number of states, nonlinearity, complexity) high-order multi-dimensional chaotic system can be used. For example, Paar [80] suggests a second order differential equation describing a robot model

$$
\begin{array}{r}
m \frac{d^{2} x}{d t^{2}}-\beta_{2}\left(\frac{d x}{d t}\right)^{2} \operatorname{sign}\left(\frac{d x}{d t}\right)-\gamma_{2} \operatorname{sign}\left(\frac{d x}{d t}\right)-\delta_{21} x-\delta_{23} x^{2}= \\
=L \frac{\omega_{0}^{2}}{2 \pi} \cos \left(\omega_{0} t\right)-\zeta_{21} e^{\lambda_{21} t}-\zeta_{22} e^{\lambda_{22} t}
\end{array}
$$

which corresponds to a hard spring with coefficients $\delta_{21}$ and $\delta_{23}$ with two types of friction. The right side of the equation represents a periodic time-dependent force with the amplitude $L\left(\omega_{0}^{2} / 2 \pi\right)$ and a feedback force given by the corresponding parameters.

To date, no such systems have been implemented as a working encryption algorithm (at least no algorithm is known to the author). This is principally due to the relatively complex numerical integration schemes that are required and the non-uniform distribution of state variables.

\subsubsection{Multi-Stream Generators}

Protopopescu [82] proposes an encryption scheme based on multiple iterated functions: $m$ different chaotic maps are initialized using a secret key. If the maps depend on parameters, these too are determined by the key. The maps are iterated using floating point arithmetic and $m$ bytes are extracted from their floating point representations, one byte from each map. These $m$ numbers are then combined using an 


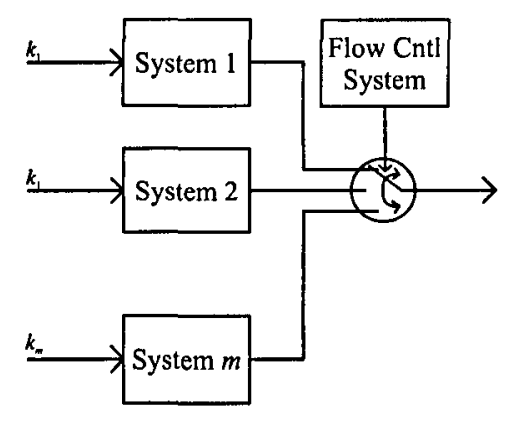

Figure 4.15: A multi-stream cryptographic generator

XOR operation. The process is repeated to create a one time pad which is finally XOR-ed with the plain-text.

In this thesis, we extend the Protopopescu scheme as follows:

1. Chaotic systems can be connected to each other (i.e. the state of each system influences the states of all other systems) to increase the average orbit length. Linked systems form a single chaotic system with a large state space and more stable orbits. A connection between chaotic systems may be established at a given time interval.

2. The set of chaotic systems (iterated functions) can be different for each encryption session. This can be implemented by supplying an iterated function set with the key.

3. The output bit can be generated in each $q$-th iteration. This increases the independence of bits as suggested in Section 3.6.

4. Chaotic systems can be permuted in a complex manner, particulary, the order of 'switching on' the systems may depend on the key (Figure 4.15).

We can define this extended cryptographic system as

$$
\begin{cases}x_{n+1}^{1}=f_{1}\left(x_{n}^{2}, k^{1}\right), & b_{j}^{1}=\sigma_{1}\left(x_{q j}^{1}\right) \\ x_{n+1}^{2}=f_{2}\left(x_{n}^{2}, k^{2}\right), & b_{j}^{2}=\sigma_{2}\left(x_{q j}^{2}\right) \\ \cdots & \cdots \\ x_{n+1}^{m}=f_{m}\left(x_{n}^{m}, k^{m}\right), & b_{j}^{m}=\sigma_{m}\left(x_{q j}^{m}\right)\end{cases}
$$




$$
b_{j}=b_{j}^{1} \oplus b_{j}^{2} \oplus \ldots \oplus b_{j}^{m}
$$

where

$f_{1}, f_{2}, \ldots, f_{m}$ are iterated functions of the session set $\left\langle x_{0}^{1}, k^{1}, x_{0}^{2}, k^{2}, \ldots, x_{0}^{m}, k^{m}\right\rangle$ are initial conditions; $b_{j}^{1}, b_{j}^{2}, \ldots b_{j}^{m}$ are the internal state bits in $(n=q j)$-th moment of time; $b_{j}$ is the generator output.

A mixing component providing the property (1) is given by

$$
\left\{\begin{array}{l}
x_{n}^{1}=\operatorname{mix}_{1}\left(x_{n}^{1}, x_{n}^{2}, \ldots, x_{n}^{m}\right) \\
x_{n}^{2}=\operatorname{mix}_{2}\left(x_{n}^{1}, x_{n}^{2}, \ldots, x_{n}^{m}\right) \\
\cdots \\
x_{n}^{m}=\operatorname{mix}_{m}\left(x_{n}^{1}, x_{n}^{2}, \ldots, x_{n}^{m}\right)
\end{array}\right.
$$

A pseudo-random generator based on multiple chaotic systems with extended properties (1) - (3) (see page 80) has been implemented in this thesis and forms the basis of the software system E-Larm (see Chapter 5 ).

For $m=4$ and $q>15$ the output sequence is statistically close to a random sequence. Nevertheless, compared with traditional generators, this solution is rather cumbersome and inefficient. It attempts to solve the problems related to the floatingpoint arithmetic in a 'extensive' way, providing $(m-1)$ redundant systems (in case one or more systems generate short orbits).

\subsubsection{Iteration Counting Ciphers}

Baptista [22] and Wong [103] propose a few encryption methods in which the ciphertext is the number of iterations. The state space $X$ of a chaotic system is partitioned into $m$ disjoint regions $\left\{X_{1}, X_{2}, \ldots, X_{m}\right\}$ (covering the entire $X$ or, possibly, part of it). A unique plaintext symbol $p$ is assigned to each region. Seeded with an initial condition ( $x_{0}$, control parameters), the system performs $n_{0}$ iterations. All the plaintexts are then encrypted into a sequence of integers

$$
c_{i}=n_{i}-n_{i-1}, \quad i=1,2, \ldots,
$$

where $n_{i}$ is the number of iterations such as the plaintext $p_{i}=\sigma\left(X_{i}\right)$ and $f^{n_{i}} \in X_{i}$. Figure 2.4-b shows the concept of iteration counting ciphers. This encryption scheme 
can be extended with a pseudo-random $n_{\min }$, generated for each plaintext symbol. The integer $n_{\min }$ defines the minimal number of iteration the system should perform before encrypting the next symbol. Clearly, the disadvantages are: (i) a probability of overflowing the counter $c_{i}=n_{i}-n_{i-1}$ (i.e. the length of a trajectory segment can be larger then the maximal number fitting the fixed-length ciphertext $c_{i}$ ); (ii) the ciphertext being larger than the plaintext; (iii) lots of redundant cycles when a long orbit occurs.

Ho [57] continued the research of Baptista and Wong, and went on to develop a software implementation of the encryption techniques, performance evaluation and cryptanalysis. Ho suggests using 4 plaintext bit blocks to speed up the cryptographic algorithm. However, this increases ciphertext redundancy -4 bits of plaintext are encrypted into a 10 bit ciphertext block. Ho discusses various chaos-specific cryptography issues - dynamic mapping from plaintext to floating-point state, trajectory distribution and algorithm complexity.

\subsubsection{Multi-valued Chaotic Maps with Analytical Solutions}

A solvable system has an analytical solution of each trajectory, i.e. for any discrete moment of time $n$ we can calculate the state $x_{n}$ from the initial condition $x_{0}$ (without performing $n$ iterations). We have already considered analytical solutions for the logistic (Section 4.2.3) and the tent (Section 4.2.4) systems.

Clearly, an analytical solution of a dynamic system is important in simulation sciences since it eliminates the rounding accumulation problem and speeds up computations. Figure 4.4 gives two trajectories of the logistic system computed using the iterated function (4.4) and the analytical solution (4.5). A special class of solvable systems is very interesting for cryptographic applications. These are one-step unpredictable solvable systems, described by Gonzalez et al. [51].

All known solutions for a chaotic system can be written in the general form

$$
x_{n}=\Psi\left(\theta T \kappa^{n}\right),
$$

where $\Psi(t)$ is a periodic function with period $T, \kappa$ is an integer and $\theta$ is a real 


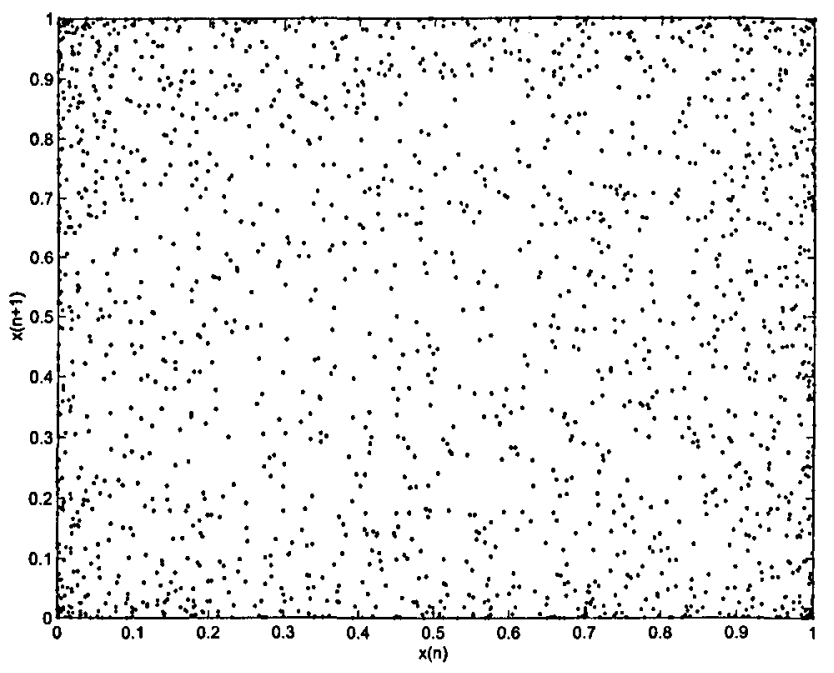

Figure 4.16: A multiple valued chaotic map $x_{n+1}=f\left(x_{n}\right)$

parameter defining the initial condition:

$$
x_{0}=\Psi(\theta T) .
$$

The system is chaotic if the Lyapunov exponent $\lambda=\ln \kappa>0$.

Consider a dynamic system with the solution

$$
x_{n}=\sin ^{2}\left(\pi \theta \kappa^{n}\right) .
$$

The one-step map $x_{n} \rightarrow x_{n+1}$ for this system is given by

$$
x_{n+1}=\sin ^{2}\left(\kappa \arcsin \sqrt{x_{n}}\right) .
$$

Unlike the logistic and the tent equations, the map (4.13) can be multi-valued, in particular, when $\kappa$ is an irrational number. Figure 4.16 shows an undefined set of points obtained with the equation 4.12 for $\kappa=\pi^{1 / 3}$.

On the one hand, analytical solutions provide random access to elements of the cryptographic sequence (unlike conventional pseudo-random generators). On the other hand, the values of $x_{n-1}$ and $x_{n+1}$ cannot be computed from $x_{n}$ because of the multi-value nature of $\arcsin$ in (4.13). So the system is one-step unpredictable (provided the adversary cannot computer the seed and/or does not know the solution). 


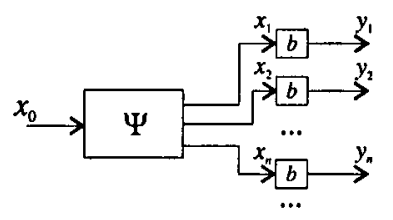

Figure 4.17: A pseudo-random generator based on an analytic solution.

The function $x_{n}=\Psi(\theta, \kappa, n)$ must have the one-way property to protect the seed $x_{0}$. The equation 4.12 has infinitely many solutions for $\theta$ and $\kappa$ given $x_{n}$. However, it is difficult to prove that given a long sequence $x_{n}, x_{n+1}, \ldots$ it is computationally impossible to recover $\theta$ (or $x_{0}$ ) and $\kappa$. To ensure this, we can introduce a hard-core predicate as illustrated in Figure 4.17.

Unfortunately, analytical solutions have the 'stretch-and-folder' property: first, the arguments $(\theta, \kappa)$ are stretched over a large space (by raising in power and multiplying), and then are folded by means a periodical function (sin, mod). Consequently, the maximum length and the 'pseudo-random' quality of the sequence is limited by the computer precision. As $n$ increases, the effect of an intermediate rounding in (4.12) becomes more destructive, so solvable systems also have a 'horizon' of predictability. For large $n$, the result (in the context of patterns and probability distributions) may be even worse than iterative computation.

Another problem is that we know analytical solutions only for certain (boundary) values of control parameters. This does not allow to use parameters as a secret key, all the secrecy must be kept in the seed.

Kotulski et al. [68] studied floating-point implementations of solvable chaotic systems (4.13) and found them useful for producing short chaotic sequences (e.g. in block ciphers), whereas long sequence did not pass statistical tests. The problem is that generators are very sensitive to the choice of seeds and control parameters, i.e. not all seeds produce a sufficiently long sequence.

\subsection{Binary Pseudo-Chaos}

Section 2.2.7 provided an introduction to binary chaos, defined by a binary iterated function $f: \Omega \rightarrow \Omega$ and a set of infinite strings $\Omega$. 
Cryptographic systems use finite strings and have a limited exponential divergence of trajectories; in our notation, they are pseudo-chaotic. Typically, binary pseudo-chaotic systems behaves similarly to chaos during the first iterations (e.g. block ciphers, where the number of rounds is, typically, $n=16$ ) until the state is truncated. After this, one can discover non-chaotic properties such as periodicity.

A common definition of the Lyapunov exponent for binary systems $\langle\mathcal{A}, f\rangle$, over a finite state space $\mathcal{A}^{m} \subset \Omega$ is given by

$$
\lambda\left(x_{0}\right)=\lim _{n \rightarrow \infty} \frac{1}{n} \log d_{H}\left(f^{n}\left(x_{0}\right), f^{n}\left(x_{0}^{\prime}\right)\right),
$$

where $x_{0}^{\prime} \in \mathcal{A}$ is taken from the neighborhood of $x$, i.e. $d_{H}\left(x_{0}, x_{0}^{\prime}\right)=1$.

In a 'good' pseudo-chaotic system of the size $2^{m}$, two trajectories beginning at adjacent points $x_{0}$ and $x_{0}^{\prime}$ diverge, on average, exactly to $\left\langle d_{H}\right\rangle=m / 2$ (Figure 4.3-b).

Like any finite-state system, a binary pseudo-chaotic system is periodical. For example, a Linear Feedback Shift Register (LFSR) given by

$$
x_{n}=\left(c_{1} x_{n-1}+c_{2} x_{n-2}+\cdots+c_{k} x_{n-k}\right) \bmod 2
$$

with particular coefficients $c_{i}[86]$ has an ideal trajectory of length $2^{m}$.

\subsubsection{RANROT}

Fog [43] introduced a class of pseudo-chaotic number generators, whose cycle lengths depend on the initial condition. This class of pseudo-chaotic system (named RANROT) is similar to additive generators but with extra rotation or swapping of bits. For example,

$$
x_{n}=\left(\left(x_{n-j}+x_{n-k}\right) \bmod 2^{m}\right) \operatorname{rotr} r,
$$

where $x_{n} \in\{0,1\}^{m}, r \in[0, m / 2)$ and $j, k$ are different integers chosen according to certain rules. This nonlinear transformation insures exponential divergence at the beginning of trajectories and unpredictable behavior if the rotation parameter $r$ is unknown. According to the author, the RANROT generators have passed all conventional tests of pseudo-randomness and appear to be faster than other generators of similar quality.

To avoid short cycles, Fog suggests making a periodicity test (up to 1000 iterations) before using a cryptographic application. 


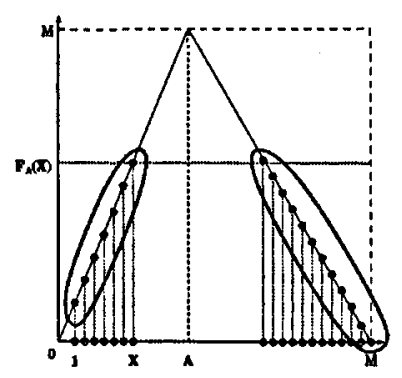

Figure 4.18: The discrete tent map.

\subsubsection{Discrete Tent Map}

Masuda et al. [75] suggest a cryptosystem based on a discretized one-dimension tent map

$$
F(X)=\left\{\begin{array}{ll}
\left\lceil\frac{M}{A} X\right\rceil, & 1 \leq X \leq A \\
\left\lfloor\frac{M}{M-A}(M-X)+1\right\rfloor, & A<X \leq M
\end{array},\right.
$$

where $A=1,2, \ldots, M$ and \lfloor\rfloor$,\lceil\rceil$ are round-down and round-up respectively. An initial condition is the plaintext; the final state is the ciphertext and a single parameter is the key. The chaotic map is applied sufficiently many times. The author discusses such properties as the correlation between invariant measures of plaintexts and ciphertexts. Sufficient conditions are given under which the cipher is resistant to differential and linear analysis.

\subsubsection{Cellular Automata}

Wolfram [102] describes a PRNG based on a one-dimensional cellular automata (CA). The system state is defined by a linear array of elements (cells)

$$
\mathbf{b}=(b(1), b(2), \ldots, b(n)) \in\{0,1\}^{n}
$$

The iterated function $f:\{0,1\}^{m} \rightarrow\{0,1\}^{m}$ is given by

$$
b(i)=b(i)-1 x \operatorname{xor}(b(i) \text { or } b(i+1)), \quad \text { for all } 1 \leq i \leq m .
$$

The array is considered circular i.e $b(m+1)=b(1)$, and the new element values are considered to be updated in parallel. The output is taken from one cell 

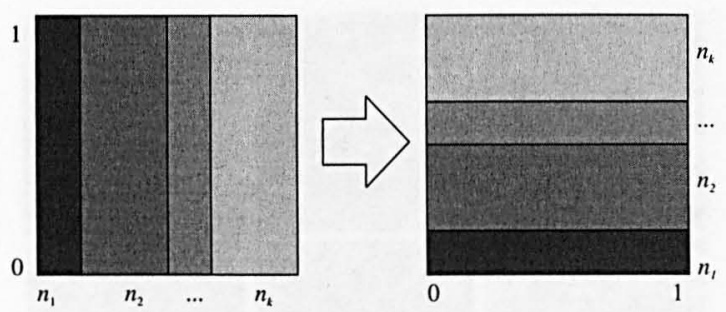

Figure 4.19: A stretch-and-fold baker transformation.

$b_{k}, k \in[0, n]$ only. This particular CA method requires three array-access operations plus two logical operations for each element of the array. While fairly simple to implement in hardware (since hardware may perform each cell computation in parallel), a large CA system may involve more computations than desired for a software implementation using a single CPU [86].

Gutowitz [52] suggests a complex scheme using a cellular automata. A 512-bit plaintext block is encrypted into a slightly larger ciphertext (578 bits). A two part key (1088-bit) is used for a particular encryption scheme with a so-called block-link structure. There are two rounds, each round consists of two subrounds and each subround includes substitution and permutation phases. The author expects that a sufficient number of rounds ensures a distribution that is statistically close to that of a random process and shows that the cipher is resistant to differential cryptanalysis.

\subsubsection{Generalized Baker Map}

A generalized Baker map, also known as Kolmogorov flows, represents the most unstable class of chaotic systems, which can be particularly useful for mixing two dimensional data blocks. The iterated function $T_{\rho}$ can be considered as a geometrical transformation of a square image: the image is divided into vertical strips according to a partition set $\rho=\left\{n_{1}, n_{2}, \ldots, n_{k}\right\}$, stretched horizontally and folded vertically as illustrated in Figure 4.19.

A discrete interpretation of the Baker map based on modular calculus is invertible. The iterated function $T_{\rho}$ is defined on a finite matrix:

$$
T_{\rho}:\{0,1\}^{N} \rightarrow\{0,1\}^{N}
$$




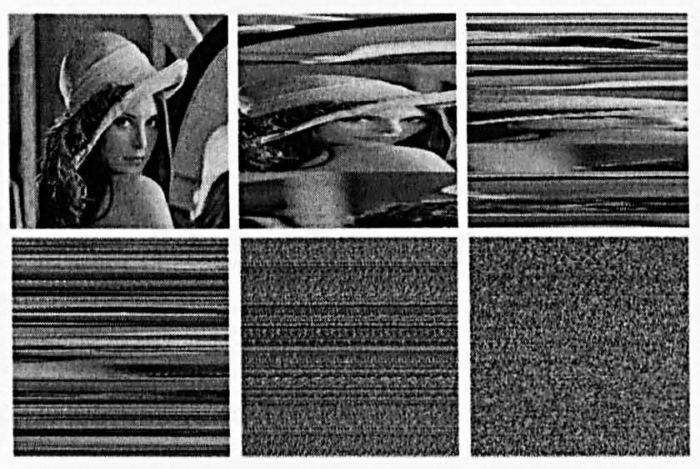

Figure 4.20: Six Baker transformations at $\rho=\{0.25,0.5,0.25\}$ applied to Lenna [89].

The set $\left\{n_{1}, n_{2}, \ldots, n_{k}\right\}$ is chosen in such a way that each integer $n_{i}$ divides $N$ and $n_{1}+n_{2}+\ldots+n_{k}=N$. The bit $b_{r, s} \in\{0,1\}^{N}$ is mapped to a new position given by

$$
T_{\rho}(r, s)=\left(\frac{N}{n_{i}}\left(r-N_{i}\right)+s \bmod \frac{N}{n_{i}}, \frac{n_{i}}{N}\left(s-s \bmod \frac{N}{n_{i}}\right)+N_{i}\right),
$$

where $r, s \in\{1,2, \ldots, N\}, N_{i}=n_{1}+n_{2}+\ldots+n_{i}, i \in\{1,2, \ldots, k\}$ and $N_{i} \leq r<N_{i}+n_{i}$. Clearly, this discrete version of the transformation becomes a key-dependent cyclic permutation.

Scharinger [89] and Fridrich [45] describe chaotic block ciphers based on discrete Kolmogorov flows. The technique appears to be highly efficient since large matrices can be permuted in bulk and relatively few iterations scramble the input object to unrecognizable form (Figure 4.20). However, this transformation should be used in combination with a substitution phase, since no permutation can change the statistical properties of the plaintext. With a simple modular addition, Fridrich extends the transformation to three dimensions. The last 3D transformation is a good substitution cipher, which produces ciphertext with a uniform histogram in a few iterations. The diffusion phase is implemented with a LFSR. The resulting encryption scheme is evaluated in terms of resistance to the general classes of attacks, and no cryptographic weakness is found. Cappelletti [32] designed a hardware implementation (using FPGA) of the Scharinger's scheme. 


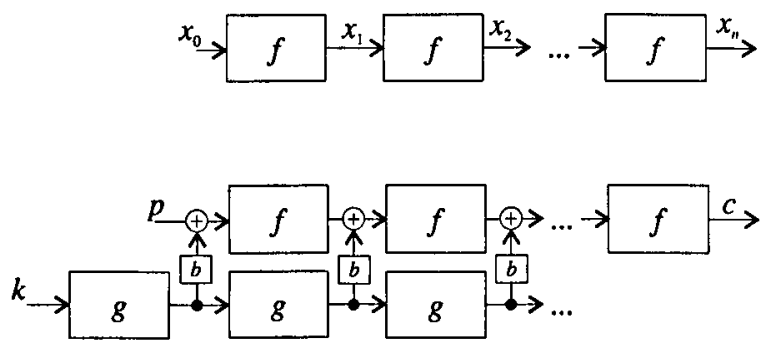

Figure 4.21: A typical block cipher is a combination of several pseudo-chaotic systems

\subsubsection{Pseudo-Chaos and Conventional Cryptosystems \\ Pseudo-Random Generators}

Existing pseudo-random generators can be viewed as pseudo-chaotic systems as illustrated in Section 3.6. Another example is the Blum-Blum-Shub system [77, 86] given by the iterated function

$$
x_{n+1}=x_{n}^{2} \bmod M,
$$

where $M=p q, p, q$ are two distinct prime numbers each congruent to 3 modulo 4 . The output bit $b_{n}$ is obtained from a predicate $\sigma\left(x_{n}\right)$, which is the last significant bit of $x_{n}$.

Besides the sensitivity to the initial condition and the topological transitivity, a pseudo-random generator has to be computationally unpredictable. The last property is ensured by a one-way iterated function and a hard-core predicate (Section 3.5.2). A one-way transformation is based on a certain mathematical problem, which is considered unsolved. For example, the Blum-Blum-Shub function works under the assumption that integer factorization is intractable.

Chaos theory is not focused on the algorithmic complexity of the iterated function, whereas in cryptography the complexity is the key issue, i.e. security. 


\section{Symmetric Block Ciphers}

All classical iterative block ciphers, in our notation, are pseudo-chaos or combinations of several pseudo-chaotic systems. As an example, consider the new Rijndael algorithm [84] accepted as $\mathrm{AES}^{2}$. The system state $x$ is a two-dimensional array of bits. The plaintext is assigned to the initial conditions $x_{0}$, and after a fixed number of iterations ( $n=10 \ldots 14$ ), the ciphertext is obtained from the final state $x_{n}$. The encryption transformation is a combination of several pseudo-chaotic maps:

1. the substitution phase is a composition of multiplicative inverse and affine transformations;

2. the mixing phase includes cycle shifts and column multiplication over a finite field;

3. the round key is obtained from another pseudo-chaotic system (pseudo-random generator).

If we consider the substitution and mixing phases as a single iterated function, the encryption scheme will represent two linked pseudo-chaotic systems (Figure 4.21).

\footnotetext{
${ }^{2}$ The Advanced Encryption Standard is an encryption algorithm for securing sensitive but unclassified material by US Government agencies and, as a likely consequence, may eventually become the de facto encryption standard for commercial transactions in the private sector.
} 


\section{Chapter 5}

\section{E-Larm: A Chaos Based Encryption System}

\subsection{Overview}

E-Larm is a cryptographic tool designed to evaluate chaos-based algorithms. It allows the generation of pseudo-chaotic sequences from a floating-point seed as well as encrypting and decrypting binary files. E-Larm provides an implementation of a multi-stream generator as described in Section 4.2.6. The encryption scheme is a sample Vernam cipher (Section 2.1.3) using a pseudo-chaotic sequence as the keystream. The software is given on the CD in Appendix A.

The analysis of the output (pseudo-chaotic sequences, ciphertext) is undertaken in mathematical packages such as Matlab 6 and Statistica 6 as well as statistical test suite developed by NIST [88].

\subsection{Algorithm}

The basic idea of this generator is to extract bits from multiple pseudo-chaotic systems in a complex and key-dependent manner. E-Larm code contains a set of one-dimensional iterated functions $f: X \rightarrow X$, all producing chaos on a certain domain (typically, $(0,1)$ ). The number of iterated function used in the same session is $\mathrm{Ns}=4$. Examples of the functions are given below (their properties are assessed 
in Chapter 6):

1. Smooth nonlinear functions

- Logistic map $x_{n+1}=p x_{n}\left(1-x_{n}\right), p=3.9$

- Sine $\operatorname{map} x_{n+1}=\left|\sin \left(p \pi x_{n}\right)\right|, p=5.0$

- Tangent-feedback map $x_{n+1}=3.3 x_{n}\left(1-\tan \left(1 / 2 x_{n}\right)\right)$

- Logarithm-feedback map $x_{n+1}=p x-n\left(1-\log \left(1+x_{n}\right)\right), p=5.0$

2. Piecewise-linear functions

- Tent map $x_{n+1}=1-\left|2 x_{n}-1\right|$

- Sawteeth map $x_{n+1}=5 x \bmod 4$

For each iterated function we define a 2-region partitioning function part : $\mathbb{X} \rightarrow$ $\{0,1\}$ (section 3.4.1) in such a way that the state point spends, on average, equal periods of time in each region. If the state point does not belong to any region, the function part returns -1. Practically, part can be defined using one of two approaches:

\section{Last Significant Bits (LSB)}

The function part extracts one or more last significant bits from the floating point format of the state variable $x$. Then the bits can be combined into one output bit, for example, using the XOR-operator. The E-Larm implementation of part extracts two last bits (for certain maps the single bit has biased statistics because of the rounding):

$$
\operatorname{part}(x)=(x \text { and } 1) \operatorname{xor}((x \operatorname{xor} 2) \gg 1)
$$

where $\gg$ is the right shift. This approach is very efficient, especially when the state space is non-uniform (e.g logistic, sine, logarithm, tangent maps) because the space is partitioned into a large number of regions lying on the resolution grid. If an area of the state space has a higher probability mass, then it is equally distributed among small regions covering this area. 


\section{Threshold Partitioning (P2)}

The second approach is to divide the state space into two large compact regions. The boundary values are selected using the symmetry of the probability density function of the system states (e.g. Figure 4.12). Symbolically, that is

$$
\operatorname{part}(x)= \begin{cases}0, & X_{m i n}^{0} \leq x \leq X_{m a x}^{0} \\ 1, & X_{m i n}^{1} \leq x \leq X_{m a x}^{1} \\ -1, & \text { otherwise }\end{cases}
$$

where $\left[X_{\text {min }}^{0}, X_{\text {max }}^{0}\right] \cup\left[X_{\text {min }}^{1}, X_{\text {max }}^{1}\right] \subset(0,1)$ and $\left[X_{\min }^{0}, X_{m a x}^{0}\right] \cap\left[X_{m i n}^{1}, X_{m a x}^{1}\right]=\emptyset$. If $X_{m a x}^{0}=X_{\min }^{1}=X_{t h r e s}$; we then have a simple threshold partitioning, or, in other words, first significant bit partitioning. Practically, this approach is more efficient for piecewise-linear maps, such as the tent or sawteeth maps, because their floating point approximation produces patterns in the last significant bits.

The following table describes variables and functions referenced in the algorithm. 


\begin{tabular}{|c|c|c|}
\hline Ns & int & number of the pseudo-chaotic systems \\
\hline MAXiter & int & $\begin{array}{l}\text { minimal and maximal number of inter- } \\
\text { mediate states }\end{array}$ \\
\hline func $[\mathrm{Ns}]$ & func [] & $\begin{array}{l}\text { set of iterated functions of the form } \\
\text { state }[s]=\text { func (state }[s], \operatorname{param}[s])\end{array}$ \\
\hline $\operatorname{part}[\mathrm{Ns}]$ & func [] & $\begin{array}{l}\text { set of partitioning functions of the form } \\
b=\text { func (state }[s] \text { ) }\end{array}$ \\
\hline $\operatorname{mix}$ & func & $\begin{array}{l}\text { mixing function of the form } \\
\text { state }[s]=\operatorname{mix}(s)\end{array}$ \\
\hline
\end{tabular}

Input Variables

$\begin{array}{lll}\mathrm{L} & \text { int } & \text { length of the output sequence } \\ \mathrm{p} \text { [Ns] } & \text { int [] } & \text { parameters of the systems } \\ \text { seed [Ns] } & \text { double [] } & \text { initial states } \\ \text { MINiter } & \text { int } & \text { minimal number of intermediate states }\end{array}$

\section{Output Variables}

out [L] byte the output sequence

\section{Internal Variables}

$\begin{array}{lll}\mathbf{s} & \text { int } & \text { counter of the systems } \\ \mathbf{i} & \text { int } & \text { counter of output symbols } \\ \mathbf{j} & \text { int } & \text { counter of intermediate states } \\ \text { state [Ns] } & \text { double [] } & \text { current states of the systems } \\ \text { b } & \text { byte } & \text { bit produced by a pseudo-chaotic sys- } \\ & & \text { tem }\end{array}$

Algorithm 5.1 describes a basic version of the E-Larm generator in a pseudocode. The generator produces a binary sequence out [L]. A combination of initial states seed [], control parameters $\mathrm{p}[\mathrm{]}$ and MINiter may be used as a secret key (generator seed). However, the values should be chosen from an appropriate range for each system.

A minimal number of chaotic iterations MINiter per round is defined to control the dependence of bits in the output sequence (see asymptotic independence in 
Section 3.6). To avoid the short cycles, at least at some degree, we suggest to mix periodically the states of pseudo-chaotic systems used. In E-Larm, the function mix is applied to the state of the $s$-th system, setting an influence of all systems on the current system:

$$
\operatorname{mix}(s)=\left(\operatorname{state}[\mathrm{s}] \sum_{q=1 \ldots N_{s}, q \neq s} \text { state [q] }\right) \bmod 1,
$$

i.e. the system state is multiplied by the sum of other system states, and the fractional part of the result is taken.

The upper limit MAXiter is necessary to treat 'bad trajectories' that come out of the chaotic attractor due to floating-point rounding. In this case the function part becomes -1 for all subsequent iterations and we reset the system with the mix function.

Typical values of parameters are given below:

$\mathrm{Ns}=4$

MINiter $=10$

MAXiter $=20$

\subsection{Practical Implementation}

\subsubsection{Platform}

The software platform of E-Larm is Java 2 version 1.4. The choice of platform was determined by the following:

- Java ensures portability of cryptographic algorithms using floating-point arithmetic. The class StrictMath contains methods for performing basic numeric operations such as the elementary exponential and trigonometric functions independently from the hardware architecture.

- Java is the most advanced developing tool of cryptographic applications. The Java Cryptography Extension (JCE) provides a framework and implementations for encryption, key generation and other algorithms.

\subsubsection{User Interface}

E-Larm has both a command line and graphical interface: 
Algorithm 5.1: Multi-Stream Pseudo-Chaotic Generator

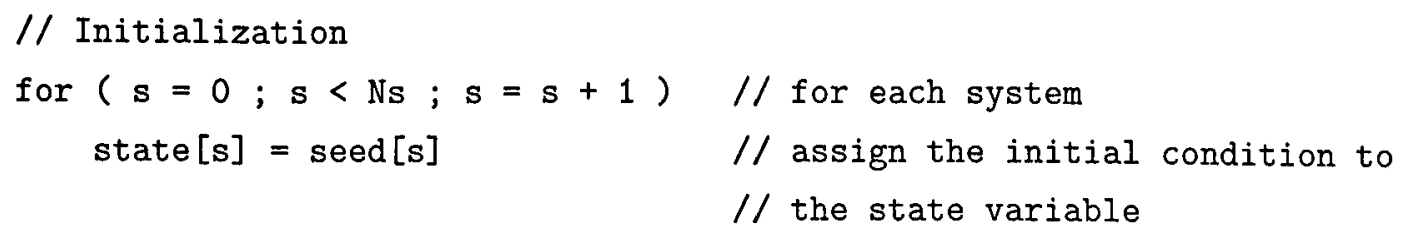

end for

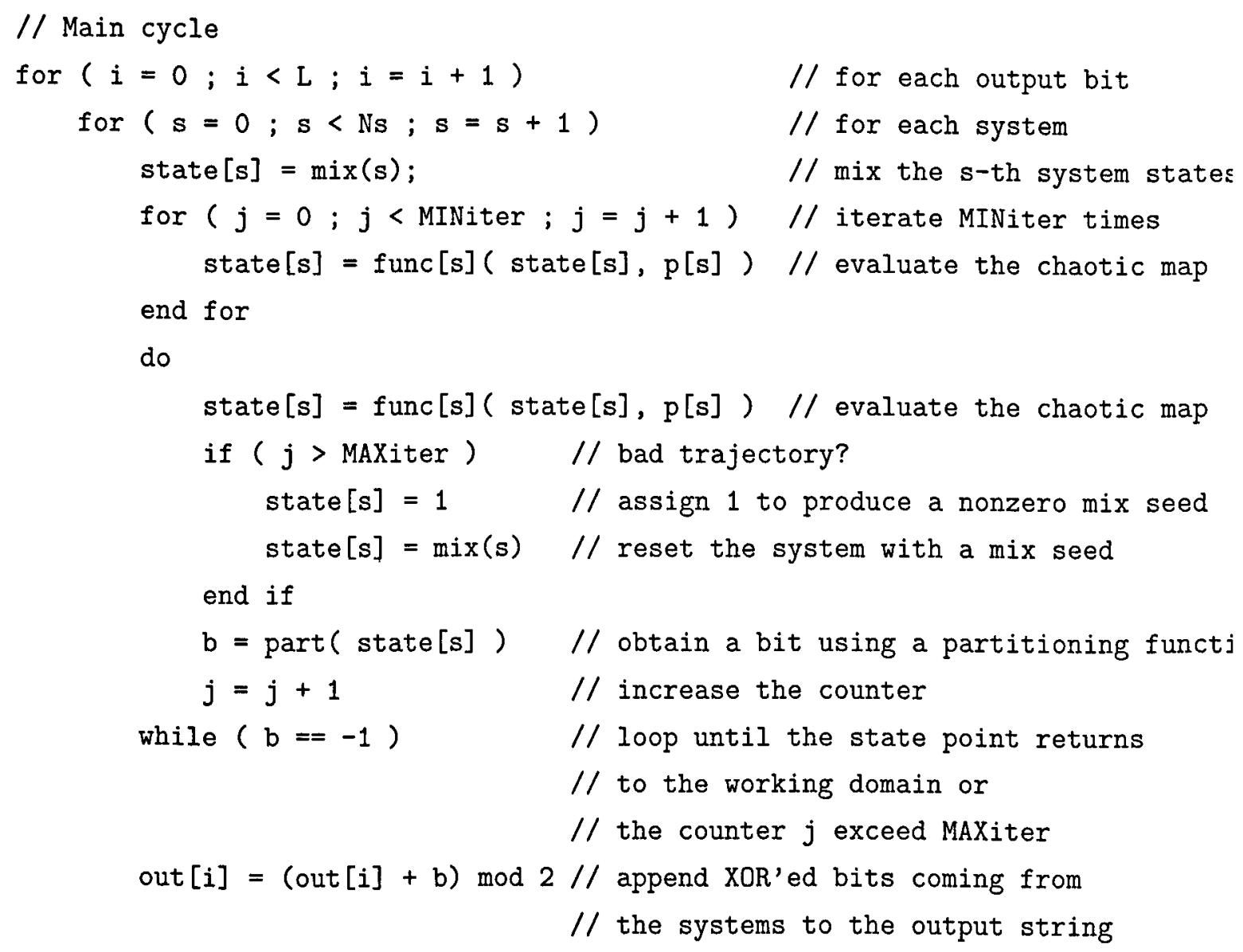




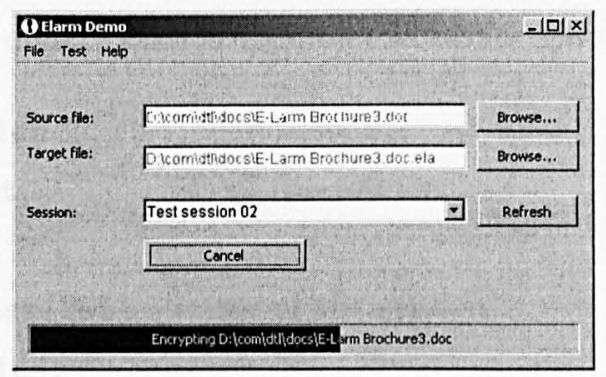

Figure 5.1: E-Larm GUI

- In command line mode, the application accepts up to 3 arguments: java ElarmDemo [InputFileName [OutputFileName [SessionName]]]

E-Larm encrypts (decrypts) the file InputFileName into InputFileName using session SessionName. If the extension of InputFileName is different from ela, the software performs encryption, otherwise (if the input file extension is ela) it performs decryption. If the number of arguments is less than 3 , the graphical user interface is lunched and initialized with arguments, if given.

- E-Larm has a graphical user interface based on the class library javax. swing. * (Figure 5.1). Input and output files can be entered manually or by means of a file browser (Browse...). Push button Encrypt (Decrypt) activates a separate thread performing the cryptographic transformation. The tread can be stopped with the button Cancel.

Additionally, one can save a pseudo-random sequence to a binary file, generated from the current session by choosing Test $\rightarrow$ Generate Session...

\subsubsection{Sessions}

An encryption (decryption) session describes a set of pseudo-chaotic systems used by the multi-stream generator. For each iterated function we define initial conditions (seed), control parameters and algorithm parameters (minimal number of iterations and a partitioning function). Session defines the environment in which the cryptographic sequence is produced and can be considered as a key. 
The following table defines session variables and their data types used by E-Larm:

\begin{tabular}{|c|c|c|}
\hline \multicolumn{3}{|c|}{ Properties of session } \\
\hline id & $\begin{array}{l}\text { int, } \\
{\left[1,2^{15}\right]}\end{array}$ & ID of the session \\
\hline name & $\operatorname{char}[255]$ & name of the session \\
\hline \multicolumn{3}{|c|}{ Properties of iterfunc } \\
\hline id & $\begin{array}{l}\text { int, } \\
{\left[1,2^{15}\right]}\end{array}$ & ID of the iterated function \\
\hline seed & $\begin{array}{l}\text { double, } \\
{[0,1]}\end{array}$ & $\begin{array}{l}\text { the initial condition of the it- } \\
\text { erated function }\end{array}$ \\
\hline miniter & $\begin{array}{l}\text { int, } \\
{\left[1,10^{4}\right]}\end{array}$ & $\begin{array}{l}\text { minimal number of intermedi- } \\
\text { ate iterations time }\end{array}$ \\
\hline $\begin{array}{l}\text { lowerbound, } \\
\text { upperbound }\end{array}$ & $\begin{array}{l}\text { double, } \\
{[0,1]}\end{array}$ & $\begin{array}{l}\text { lower and upper bounds of a } \\
\text { state space subset from which }\end{array}$ \\
\hline & & $\begin{array}{l}\text { produced. These paramet- } \\
\text { ers define the partitioning } \\
\text { function part: the interval } \\
\text { [lowerbound, upperbound] } \\
\text { is equally divided in two } \\
\text { region corresponding to logical } \\
0 \text { and } 1 \text {. This parameters are } \\
\text { ignored if the LSB partitioning } \\
\text { function is used. }\end{array}$ \\
\hline
\end{tabular}

For the purpose of the usability, sessions are stored in the XML format as illustrated below. Once the E-Larm application is started, sessions are loaded from conf/sessions . xml and becomes available in the Session combo box (Figure 5.1). The Refresh button causes the sessions to be reloaded from the file. 
Source 5.1: An XML session file

$<$ ?xml version="1.0" encoding="UTF-8"?>

$<$ !-- Elarm Session Keys -->

<!DOCTYPE sessions SYSTEM "sessions.dtd">

〈sessions>

<session id="1" name="Test Session 01">

<iterfunc id="1" seed="0.26" miniter $=" 10 "$

lowerbound="0.2" upperbound="0.8"/>

<iterfunc id="2" seed="0.33" miniter="12"

lowerbound="0.18" upperbound="0.9"/>

<iterfunc id="3" seed="0.745" miniter="8"

lowerbound="0.6" upperbound="1"/>

$</$ session $>$

<session id="2" name="Test Session 02">

<iterfunc id="2" seed="0.87" miniter="12"

lowerbound="0.18" upperbound="0.9"/>

<iterfunc $i d=" 3 "$ seed="0.61" miniter="8"

lowerbound="0.6" upperbound="1"/>

<iterfunc id="4" seed="0.589" miniter="10"

lowerbound="0.25" upperbound="0.8"/>

$</$ session $>$

$</$ sessions $>$ 


\subsection{Initial Conditions and Parameters}

Chaotic generators are sensitive to the choice of initial conditions and parameters. If the initial condition $x_{0}$ does not belong the chaotic (strange) attractor, the system can reach a point attractor or periodic attractor, and start generating a trivial sequence. It is important to ensure that the control parameters correspond a chaotic behaviour.

In E-Larm the range for the initial condition $x_{0}$ and the value of parameter $p$ were defined in the following way:

- For well known chaotic map (logistic, sine, tent, sawteeth maps), the values were taken to guarantee most unstable mode as described in the literature (e.g. [81], [20]).

- Original chaotic functions (tangent, logarithm) were assigned parameters by studying these functions analytically and by carrying simulations in Matlab. In particular, histograms (PDF of the state space) were studied at different control parameters (histogram plots are given in Section 6.5).

Following is a list of iterated functions with initial conditions and control parameters used by E-Larm. Initial conditions (seed) are defined in the session file (Section 5.3.3), whereas control parameters are built in the application (i. e. defined in the source code).

Table 5.1: Continuous nonlinear maps

\begin{tabular}{|l|l|l|l|}
\hline Name & Equation & Params & Init. Cond. \\
\hline Logistic map & $x_{n+1}=p x_{n}\left(1-x_{n}\right)$ & $p=3.99$ & $x_{0} \in(0,1)$ \\
\hline Sine map & $x_{n+1}=\left|\sin \left(p \pi x_{n}\right)\right|$ & $p=5.0$ & $x_{0} \in(0,1)$ \\
\hline Tangent-feedback map & $x_{n+1}=p x_{n}\left(1-\tan \left(0.5 x_{n}\right)\right)$ & $p=3.3$ & $x_{0} \in(0.5,1.5)$ \\
\hline Logarithm-feedback map & $x_{n+1}=p x_{n}\left(1-\log \left(1+x_{n}\right)\right)$ & $p=5.0$ & $x_{0} \in(0.3,1.5)$ \\
\hline Exactly solvable map & $x_{n}=\sin ^{2}\left(\pi \theta \kappa^{n}\right)$ & $\kappa=\pi^{1 / 3}$ & $x_{0}=(0,1)$ \\
\hline
\end{tabular}


Table 5.2: Piecewise-linear maps

\begin{tabular}{|l|l|l|l|}
\hline Name & Equation & Params & Init. Cond. \\
\hline Tent map & $x_{n+1}=1-\left|p x_{n}-1\right|$ & $p=2$ & $x_{0} \in(0,1)$ \\
\hline Sawteeth map & $x_{n+1}=p / q x_{n} \bmod q$ & $p=5, q=4$ & $x_{0} \in(0,1)$ \\
\hline
\end{tabular}

\subsection{Evaluation}

Pseudo-randomness and performance tests are described in Chapter 6. 


\section{Chapter 6}

\section{Evaluation of}

\section{Chaos-based PRNG's used for E-Larm}

\subsection{Introduction}

Various statistical tests $[64,77,85,88,74,98]$ can be applied to a given chaotic sequence to compare and evaluate it to a truly random sequence (section 3.3.1). The likely outcome of statistical tests, when applied to a truly random sequence, is known a priori and can be described in probabilistic terms. There are an infinite number of possible statistical tests, each assessing the presence or absence of a pattern which, if detected, would indicate that the sequence is non-random, but no specific finite set of tests is deemed 'complete'. In addition, the results of statistical testing must be interpreted with some care and caution to avoid incorrect conclusions about a specific generator.

In this research we borrow the test methodology described in [88]. A classical statistical test is formulated to test a specific null hypothesis $H_{0}$. Let the null hypothesis be that the sequence being tested is random. Associated with this null hypothesis is the alternative hypothesis $H_{a}$, which is that the sequence is not random. For each applied test, a decision or conclusion is derived that accepts or rejects the null hypothesis, i.e., whether the generator is (or is not) producing random values, 
based on the sequence that was produced.

For each test, a relevant randomness statistic must be chosen and used to determine the acceptance or rejection of the null hypothesis. Under an assumption of randomness, such a statistic has a distribution of possible values. A theoretical reference distribution of this statistic under the null hypothesis is determined by mathematical methods. From this reference distribution, a critical value is determined (typically, this value is 'far out' in the tails of the distribution, say out at the $99 \%$ point). During a test, a test statistic value is computed on the data (the sequence being tested). This test statistic value is compared to the critical value. If the test statistic value exceeds the critical value, the null hypothesis for randomness is rejected. Otherwise, the null hypothesis (the randomness hypothesis) is not rejected (i.e. the hypothesis is accepted).

In practice, the reason that statistical hypothesis testing works is that the reference distribution and the critical value are dependent on and generated under a tentative assumption of randomness. If the randomness assumption is, in fact, true for the data at hand, then the resulting calculated test statistical value on the data will have a very low probability (e.g., $0.01 \%$ ) of exceeding the critical value.

On the other hand, if the calculated test statistical value does exceed the critical value (i.e., if the low probability event does in fact occur), then from a statistical hypothesis testing point of view, the low probability event should not occur naturally. Therefore, when the calculated test statistical value exceeds the critical value, the conclusion is made that the original assumption of randomness is suspect or faulty. In this case, statistical hypothesis testing yields the following conclusions: reject $H_{0}$ (randomness) and accept $H_{a}$ (non-randomness).

Statistical hypothesis testing is a conclusion-generation procedure that has two possible outcomes, either accept $H_{0}$ (the data is random) or accept $H_{a}$ (the data is non-random). The following table relates the true (unknown) status of the data at hand to the conclusion arrived at using the testing procedure: 


\begin{tabular}{|c|c|c|}
\hline \multirow{2}{*}{ True situation } & \multicolumn{2}{|c|}{ Conclusion } \\
\cline { 2 - 3 } & Accept $H_{0}$ & Accept $H_{a}$ \\
\hline Data is random $\left(H_{0}\right.$ is true $)$ & No error & Type I error \\
\hline Data is not random $\left(H_{a}\right.$ is true $)$ & Type II error & No error \\
\hline
\end{tabular}

If the data is, in truth, random, then a conclusion to reject the null hypothesis (i.e., conclude that the data is non-random) will occur a small percentage of the time. This conclusion is called a Type I error. If the data is, in truth, non-random, then a conclusion to accept the null hypothesis (i.e., conclude that the data is actually random) is called a Type II error. The conclusions to accept $H_{0}$ when the data is really random, and to reject $H_{0}$ when the data is non-random, are correct.

The probability of a Type I error is often called the level of significance of the test. This probability can be set prior to a test and is denoted as $\alpha$ (ALPHA). For the test, $\alpha$ is the probability that the test will indicate that the sequence is not random when it really is random. That is, a sequence appears to have non-random properties even when a 'good' generator produced the sequence. Common values of $\alpha$ in cryptography are about 0.01 .

The probability of a Type II error is denoted as $\beta$. For the test, $\beta$ is the probability that the test will indicate that the sequence is random when it is not; that is, a 'bad' generator produces a sequence that appears to have random properties. Unlike $\alpha, \beta$ is not a fixed value. $\beta$ can take on many different values because there are an infinite number of ways that a data stream can be non-random, and each different way yields a different $\beta$. The calculation of the Type II error $\beta$ is more difficult than the calculation of a because of the many possible types of non-randomness.

One of the primary goals of the following tests is to minimize the probability of a Type II error, i.e., to minimize the probability of accepting a sequence being produced by a good generator when the generator was actually bad. The probabilities $\alpha$ and $\beta$ are related to each other and to the size $N$ of the tested sequence in such a way that if two of them are specified, the third value is automatically determined. In practice, we usually select a sample size $N$ and a value for $\alpha$. Then a critical point for a given statistic is selected that will produce the smallest $\beta$. That is, a suitable sample size is selected along with an acceptable probability of deciding that 
a bad generator has produced the sequence when it really is random. Then the cutoff point for acceptability is chosen such that the probability of falsely accepting a sequence as random has the smallest possible value.

Each test is based on a calculated test statistic value, which is a function of the data. If the test statistic value is $S$ and the critical value is $t$, then the Type I error probability is $P\left(S>t \mid H_{0}\right.$ is true $)=P$ (reject $H_{0} \mid H_{0}$ is true), and the Type II error probability is $P\left(S \leq t \mid H_{0}\right.$ is false $)=P$ (accept $H_{0} \mid H_{0}$ is false). The test statistic is used to calculate a $P$-value that summarizes the strength of the evidence against the null hypothesis. For these tests, each $P$-value is the probability that a perfect random number generator would have produced a sequence 'less random' than the sequence that was tested, given the kind of non-randomness assessed by the test.

If a $P$-value for a test is determined to be equal to 1 , then the sequence appears to have perfect randomness. A $P$-value of zero indicates that the sequence appears to be completely non-random. A significance level $\alpha$ can be chosen for the tests. If $P$-value $\geq \alpha$, then the null hypothesis is accepted; i.e., the sequence appears to be random. If $P$-value $<\alpha$, then the null hypothesis is rejected; i.e., the sequence appears to be non-random. The parameter $\alpha$ denotes the probability of the Type I error. Typically, $\alpha$ is chosen in the range $[0.001,0.01]$.

Most of the tests have the standard normal and the chi-square $\chi^{2}$ as reference distributions. If the sequence under test is in fact non-random, the calculated test statistic will fall in extreme regions of the reference distribution. The standard normal or Gaussian distribution

$$
f(x, \mu, \sigma)=\frac{1}{\sqrt{2 \pi \sigma^{2}}} e^{-\frac{1}{2}\left(\frac{x-\mu}{\sigma}\right)^{2}}
$$

is used to compare the value of the test statistic obtained from the RNG with the expected value of the statistic under the assumption of randomness. The test statistic for the standard normal distribution is of the form $z=(x-\mu) / \sigma$, where $x$ is the sample test statistic value, and $\mu$ and $\sigma^{2}$ are the expected value and the variance of the test statistic. The $\chi^{2}$ distribution is used to compare the goodnessof-fit of the observed frequencies of a sample measure to the corresponding expected frequencies of the hypothesized distribution. The test statistic is of the form $\chi^{2}=$ $\sum\left(\left(o_{i}-e_{i}\right)^{2} / e_{i}\right)$, where $o_{i}$ and $e_{i}$ are the observed and expected frequencies of occurrence of the measure, respectively. 


\subsection{Considerations for Randomness and Unpredictability}

The following assumptions are made with respect to random binary sequences to be tested:

Uniformity: At any point in the generation of a sequence of random or pseudorandom bits, the occurrence of a zero or one is equally likely, i.e., the probability of each is exactly $1 / 2$. The expected number of zeros (or ones) is $N / 2$.

Scalability: Any test applicable to a sequence can also be applied to subsequences extracted at random. If a sequence is random, then any such extracted subsequence should also be random. Hence, any extracted subsequence should pass any test for randomness.

Consistency: The behavior of a generator must be consistent across initial conditions (seeds). It is inadequate to test a PRNG based on the output from a single seed, or a generator on the basis of an output produced from a single physical output.

\subsection{Chaotic Maps}

The following tables describe chaotic maps that have been tested. The finite state approximation was the 64-bit floating-point format (double). Two different partitioning methods were used: last significant bit (LSB) for continuous nonlinear maps, and threshold partitioning ( 2 regions) for piecewise-linear maps (see sections 5.2 and 3.4.1). This choice was determined experimentally as will be discussed later. 
Table 6.1: Continuous nonlinear maps

\begin{tabular}{|l|l|l|l|l|}
\hline Name & Code & Equation & Params & Fig. \\
\hline Logistic map & par & $x_{n+1}=p x_{n}\left(1-x_{n}\right)$ & $p=3.99$ & 6.1 \\
\hline Sine map & $\sin$ & $x_{n+1}=\left|\sin \left(p \pi x_{n}\right)\right|$ & $p=5.0$ & 6.3 \\
\hline Tangent-feedback map & $\tan$ & $x_{n+1}=p x_{n}\left(1-\tan \left(0.5 x_{n}\right)\right)$ & $p=3.3$ & 6.4 \\
\hline Logarithm-feedback map & $\log$ & $x_{n+1}=p x_{n}\left(1-\log \left(1+x_{n}\right)\right)$ & $p=5.0$ & 6.5 \\
\hline Exactly solvable map & exactsol & $x_{n}=\sin ^{2}\left(\pi \theta \kappa^{n}\right)$ & $\kappa=\pi^{1 / 3}$ & 6.9 \\
\hline
\end{tabular}

Table 6.2: Piecewise-linear maps

\begin{tabular}{|l|l|l|l|l|}
\hline Name & Code & Equation & Params & Fig. \\
\hline Tent map & tent & $x_{n+1}=1-\left|p x_{n}-1\right|$ & $p=2$ & 6.6 \\
\hline Sawteeth map & saw & $x_{n+1}=p / q x_{n} \bmod q$ & $p=5, q=4$ & 6.7 \\
\hline
\end{tabular}

The E-Larm mixing scheme (referenced hereafter as 'combo' system) has been tested on 4 pseudo-chaotic systems: logistic, sine, tangent and sawteeth maps.

\subsection{Visual Approaches}

Before starting numeric tests it is helpful to check the PRNG behavior using simple plots in different domains: space-time, phase-space and frequency as well as to check the time series distribution.

\subsubsection{Phase-Space Diagrams}

Traditionally, a chaotic system is defined by an iterated function of the form $x_{n+1}=$ $f\left(x_{n}\right)$ as introduced in section 2.2. Most of chaotic maps, that have been studied, are simple and easily predictable (first row in Figures 6.1, 6.3, 6.4, 6.5, 6.6, 6.7, $6.9)$. However, the 15-round composition $x_{n+15}=f^{15}\left(x_{n}\right)$ shows the complexity and mixing effect of chaos. Such a multi-round transformation ensures the asymptotic independence of the output bits (section 3.6), i.e hides the time series correlations and increases the computational unpredictability. 


\subsubsection{Time series}

The second row in Figures 6.1, 6.3 etc depicts a time series and its 1000-bin histogram (approximated probability density function). Visibly, the histograms of the floatingpoint numbers are not flat, i.e. the system states are not uniformly distributed over the state space and, consequently, are predictable. However, histograms of extracted bits using a convenient partition method appear to be uniform:

- In the case of a piecewise linear map (sawteeth, tent maps) the statistical result is better when we choose two or more compact non-overlapping regions (e.g. using a threshold). Piecewise linear maps can produce time series with good randomness in higher order bits, but often keep the last bit(s) unmodified (as illustrated in Figure 6.8), even with control parameters that should theoretically guarantee chaos.

- On the other hand, for a continuous nonlinear map (logistic, sine maps), the last significant bit (LSB) method provides more uniform statistics because of a rounding effect (Figure 6.2). The LSB method works even when the histogram is not symmetric and it is difficult to find two compact partitions where total probability masses are equal (e.g. Figures 6.4 and 6.5).

The time series obtained with an analytical (exact) solution (section 4.2.8) have different histograms in the beginning and at the end of the sequence (Figure 6.9) because the precision degrades. The last approximated PDF is not continuous, which illustrates the rounding and approximating artifacts. The cycle length histogram is given in Figure 6.10.

\subsubsection{Power Spectrum}

The spectral density Fourier transform function detects periodic features. The spectral density function (spectrum) is given by

$$
P_{m}=\left(\frac{1}{N} \sum_{n=-N / 2}^{N / 2} x_{n} \exp (-i 2 \pi n m / N)\right)^{2}
$$

where $N$ is the length of the sequence, $m$ is the spatial frequency. 
It is considered that the spectrum should be uniform and no more than $5 \%$ of the peaks should surpass the $95 \%$ cutoff. Clearly, most of chaotic maps have a nonunform spectrum of their time series. But, if we drop out $k=15$ intermediate states (multi-round composition), the new sequence will have a 'more random' spectrum (bottom row of Figures 6.1, 6.3 etc).

\subsubsection{Approximated Entropy}

Approximated entropy (APEN) characteristics are based on repeating patterns in the string. "For a fixed block length $m, \operatorname{APEN}(m)$ measures the logarithmic frequency with which blocks of length $m$ that are close together remain close together for blocks augmented by one position. Thus, small values of APEN $(m)$ imply strong regularity, or persistence, in a sequence. Alternatively, large values of APEN $(m)$ imply substantial fluctuation, or irregularity" (Pincus and Singer in [88]). In a long truly random sequence, one should expect that $\operatorname{APEN}(m) \approx \log 2 \approx 0.6931$.

Figure 6.11 depicts the APEN values for five binary sequences taken from:

- the binary expansion of $\pi=3.1416 \ldots$,

- LCG, Linear Congruent Generator, (page 3.6),

- SHA1G, Secure Hash Algorithm Generator described in FIPS 186 (sha-1),

- Logistic system (par),

- Sine system $(\sin )$,

- E-Larm, 4 stream combo (mix).

The $x$-axis reflects the number of bits considered in the sequence. The $y$-axis reflects the deficit from maximal irregularity, that is, the difference between the $\log 2$ and the observed approximate entropy value. For a fixed sequence length, one can determine which sequence appears to be more random.

For a sequence of $1 \mathrm{M}$ bits, (Figure 6.11, upper plot), logistic and sine systems appear more random that LCG. On the lower plot (2M bit sequences) both E-Larm and SHA1G have higher entropy than the binary extension of $\pi$. However, for larger block sizes, this is not the case [88]. 
Figure 6.1: Properties of the logistic map

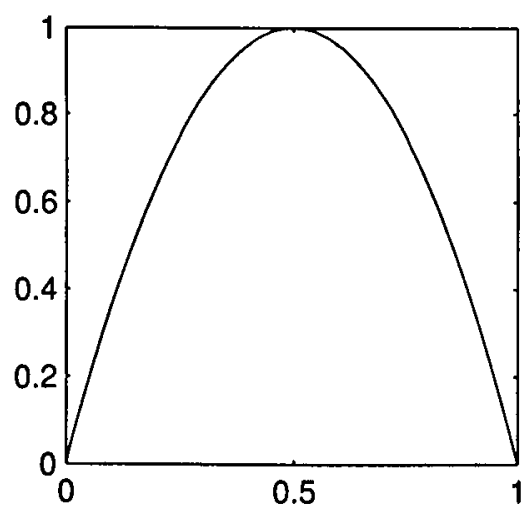

The iterated function $x_{n+1}=f\left(x_{n}\right)$

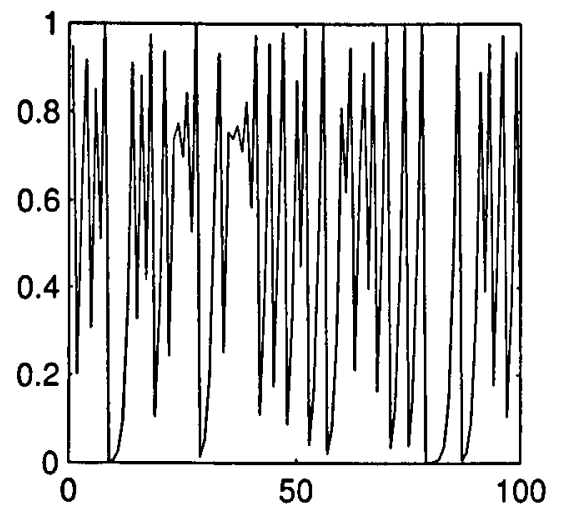

A time series (shown $N=100$ )

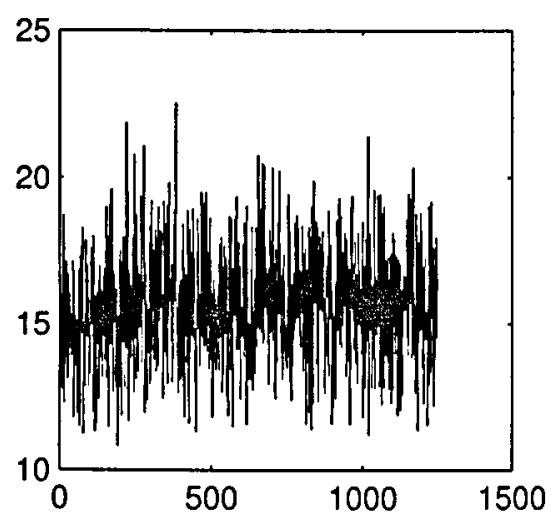

The spectral density Fourier transform of $x_{1}, x_{2}, \ldots, x_{N} \quad(N=6000)$ averaged from 10 experiments

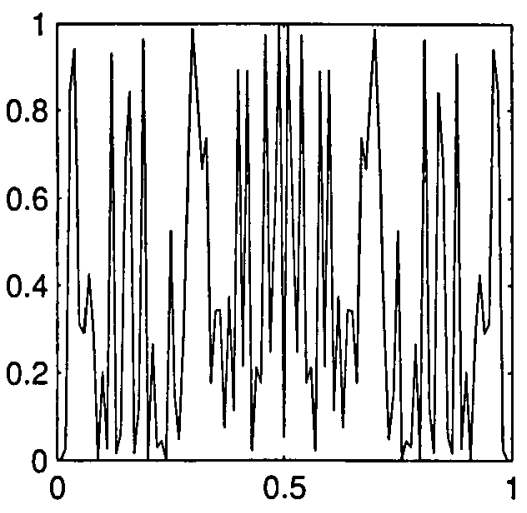

The composition of 15 iterations $x_{n+15}=f^{15}\left(x_{n}\right)$
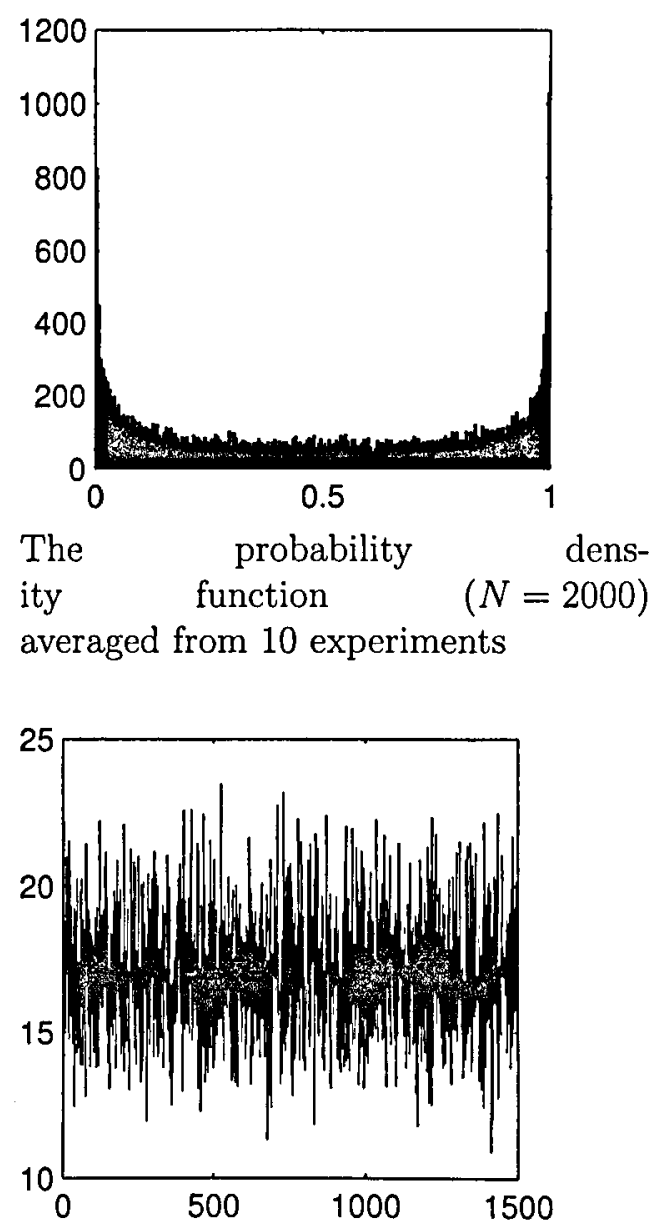

The spectral density Fourier transform of $x_{1}, x_{15}, \ldots, x_{15 N} \quad(N=6000)$ averaged from 10 experiments 


\subsection{Brief Description of Tests}

There are 16 tests in the NIST statistical package, and 12 of them have been applied to analyze pseudo-chaotic sequences. The following is their brief description and parameter values used. The test number and code name in teletype is given for further reference.

1 Frequency (monobit) test (frequency) determines whether the number of ones and zeros in a sequence are approximately the same as would be expected for a truly random sequence.

2 Frequency test within a block (block-frequency) determines whether the frequency of ones in an $M$-bit block $(M=100)$ is approximately $M / 2$, as would be expected under an assumption of randomness.

3 Runs test (runs) focuses on the total number of runs in the sequence, where a run is an uninterrupted sequence of identical bits. A run of length $k$ consists of exactly $k$ identical bits and is bounded before and after with a bit of the opposite value. Among all the runs half should be of length 1, a quarter should be of length 2, an eighth should be of length 3 and so on (as long as the number of runs so indicated exceeds one); for each of these lengths there should be equally many runs of zero bits and runs of one bits.

4 Test for the longest run of ones in a $M$-bit block (longest-run) determines whether the length of the longest run of ones within the tested sequence is consistent with the length of the longest run of ones that would be expected in a random sequence.

5 Binary Matrix Rank Test (rank) focuses on the rank of disjoint submatrices of the entire sequence. The test checks for linear dependence among fixed length substrings of the original sequence.

6 Spectral Density Test using Discrete Fourier transform ( $f \mathrm{ft}$ ) measures peak heights in the Discrete Fourier Transform (DFT) of the sequence. The intention is to detect whether the number of peaks exceeding the $95 \%$ threshold is significantly different than $5 \%$. 
7 Non-overlapping Template Matching Test (nonperiodic-templates) measures the number of occurrences of pre-specified target strings. The purpose of this test is to detect generators that produce too many occurrences of a given non-periodic (aperiodic) pattern. For this test and for the Overlapping Template Matching test, an $m=10$ bit window is used to search for a specific $m$-bit pattern. If the pattern is not found, the window slides one bit position. If the pattern is found, the window is reset to the bit after the found pattern, and the search resumes.

8 Overlapping Template Matching Test (overlapping-templates) counts the number of occurrences of prespecified target strings. Both this test and the previous test use an $m=20$ bit window to search for a specific $m$-bit pattern. The difference is that when the pattern is found, the window slides only one bit before resuming the search.

9 Maurer's 'Universal Statistical' Test (universal) focuses on the number of bits between matching patterns (a measure that is related to the length of a compressed sequence). The purpose of the test is to detect whether or not the sequence can be significantly compressed without loss of information. A significantly compressible sequence is considered to be non-random.

10 Serial Test (serial) is focused on the frequency of all possible overlapping $m=10$ bit patterns across the entire sequence. The purpose of this test is to determine whether the number of occurrences of the $2^{m} m$-bit overlapping patterns is approximately the same as would be expected for a random sequence. Random sequences have uniformity; that is, every $m$-bit pattern has the same chance of appearing as every other $m$-bit pattern.

11 Approximate Entropy Test (apen) As with the Serial test, the focus of this test is the frequency of all possible overlapping $m$-bit patterns across the entire sequence. The purpose of the test is to compare the frequency of overlapping blocks of two consecutive/adjacent lengths $(m$ and $m+1)$ against the expected result for a random sequence. 
The pseudo-chaotic systems, which have been tested, are given below with their notation code, number of rounds and length of the sequence generated:

\begin{tabular}{|c|c|c|c|c|}
\hline $\begin{array}{l}\text { Iterated } \\
\text { Function }\end{array}$ & Code & $\begin{array}{l}\text { Number of } \\
\text { Rounds }\end{array}$ & $\begin{array}{l}\text { Length of } \\
\text { Sequence }\end{array}$ & Fig. \\
\hline SHA1G & sha-1 & - & 500000 & $\begin{array}{l}6.12 \\
6.13\end{array}$ \\
\hline Logistic map & par & 15 & 500000 & $\begin{array}{l}6.12 \\
6.13\end{array}$ \\
\hline Logistic map & par-bad & 1 & 500000 & $\begin{array}{l}6.12 \\
6.13\end{array}$ \\
\hline Sine map & $\sin$ & 15 & 500000 & $\begin{array}{l}6.12 \\
6.13\end{array}$ \\
\hline $\begin{array}{l}\text { Tangent- } \\
\text { feedback } \\
\text { map }\end{array}$ & $\tan$ & 15 & 500000 & $\begin{array}{l}6.14 \\
6.15\end{array}$ \\
\hline $\begin{array}{l}\text { Logarithm- } \\
\text { feedback } \\
\text { map }\end{array}$ & $\log$ & 15 & 500000 & $\begin{array}{l}6.14, \\
6.15\end{array}$ \\
\hline $\begin{array}{l}\text { Sawteeth } \\
\text { map }\end{array}$ & saw & 15 & 500000 & $\begin{array}{l}6.14 \\
6.15\end{array}$ \\
\hline $\begin{array}{l}4 \text { stream } \\
\text { combo } \\
\text { (E-Larm) }\end{array}$ & $\operatorname{mix}$ & 16 & 500000 & $\begin{array}{l}6.14 \\
6.15\end{array}$ \\
\hline $\begin{array}{l}4 \text { stream } \\
\text { combo } \\
\text { (E-Larm) }\end{array}$ & $\operatorname{mix}$ & 16 & 2000000 & 6.16 \\
\hline
\end{tabular}

The following test procedure has been applied to the pseudo-chaotic systems:

1. Four different seeds were taken from the valid range (See Tables 5.1 and 5.2 on page 100) using a standard Java PRNG; the control parameter $p$ was assigned a constant as given in Tables 5.1 and 5.2 .

2. Four pseudo-chaotic sequence of the length $N=500,000$ (denoted as T1, T2, T3 and T4) were produced by the E-Larm PRNG.

3. The eleven tests described above were made for each sequence. The test software was compiled on a Linux platform from NIST sources [88]. 
4. $(4 \times 11) P$-value were obtained and saved in tables (Figures 6.12-6.16).

5. The average $P$-value was computed from four values.

In addition to the standard length $N=500,000$, four long sequences $(N=$ 2,000,000) were generated and tested using the mix system (E-Larm). Following is a description of the results given in Figures 6.12-6.16:

\begin{tabular}{l|l} 
TEST SUMMARY FOR <code> & $\begin{array}{l}<\text { code> reference a pseudo-chaotic sys- } \\
\text { tem (see the table above) } \\
\text { Level of significance } \alpha\end{array}$ \\
ALPHA & $\begin{array}{l}\text { Length of the sequence } \\
\text { Tn denotes a } P \text {-value column corres- } \\
\text { ponding to the n-th sequence. Four dif- } \\
\text { ferent sequence were tested } \\
\text { Average } P \text {-value } \\
\text { Test conclusion. The test fails if more } \\
\text { then one sequence has } P \text {-value }< \\
\text { The generator is considered pseudo- } \\
\text { MEAN } \\
\text { random if it passes all the tests }\end{array}$ \\
FINAL CONLUSION
\end{tabular}


Figure 6.2: Properties of the logistic map (cont.)
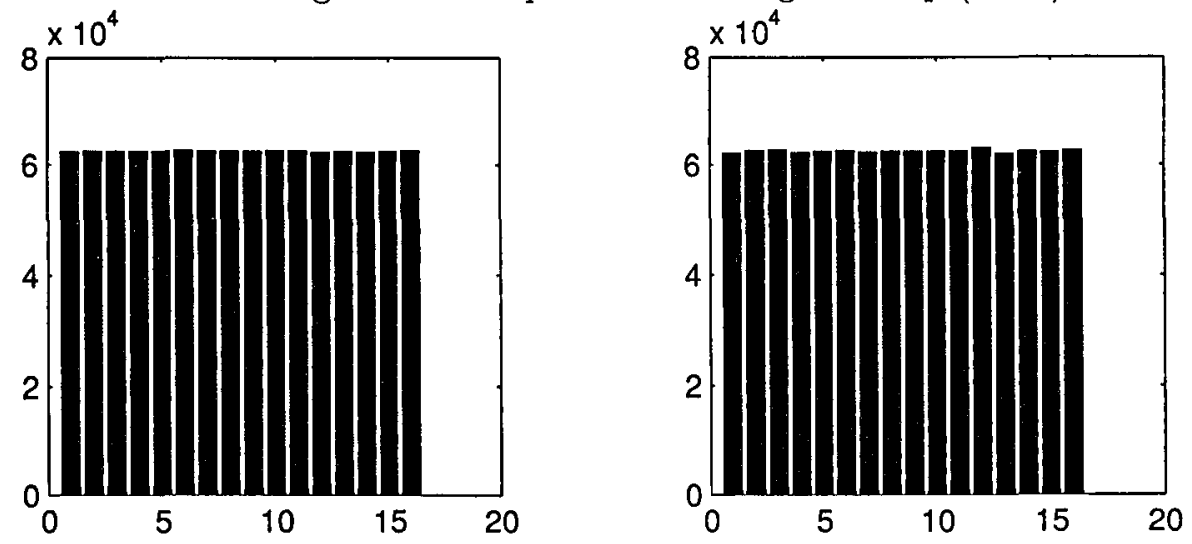

Histogram of 4-bit values obtained us- Histogram of 4-bit values obtained using XOR product of the 2 last signific- ing threshold partitioning at $x_{\text {thres }}=$ ant bits $(N=1,000,000)$ $0.552(N=1,000,000)$ 
Figure 6.3: Properties of the sine map

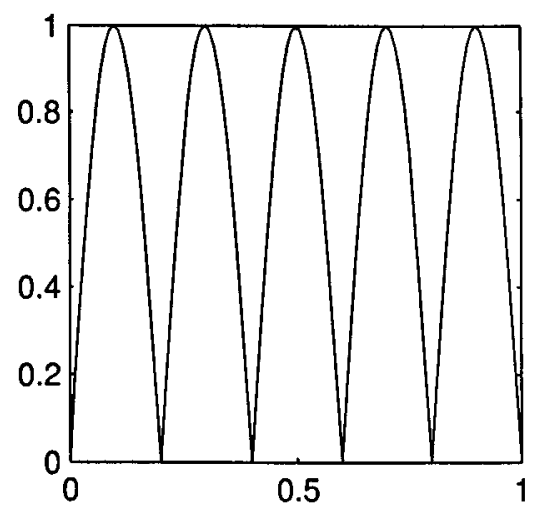

The iterated function $x_{n+1}=f\left(x_{n}\right)$

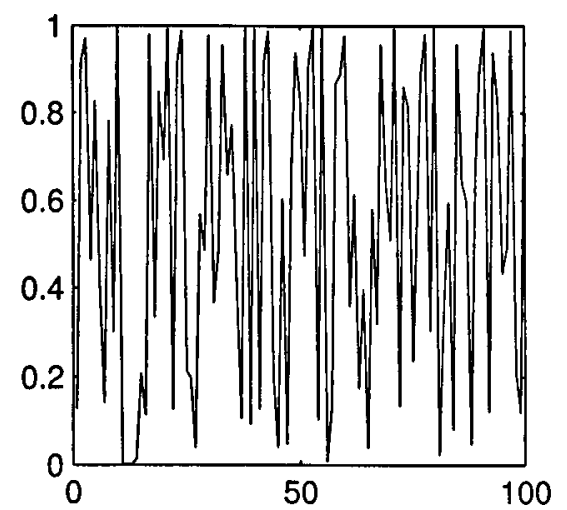

A time series (100 steps) $x_{1}, x_{2}, \ldots, x_{N}$ (shown $N=100$ )

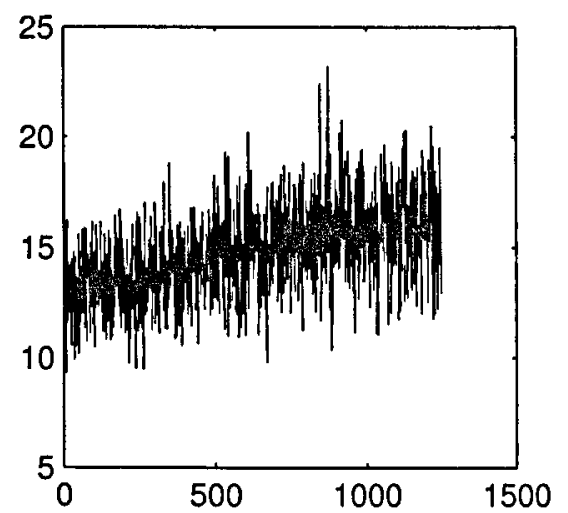

The spectral density Fourier transform of $x_{1}, x_{2}, \ldots, x_{N} \quad(N=6000)$ averaged from 10 experiments

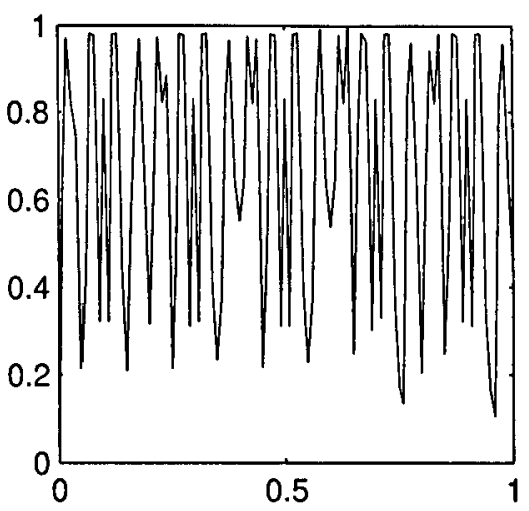

The composition of 15 iterations $x_{n+15}=f^{15}\left(x_{n}\right)$

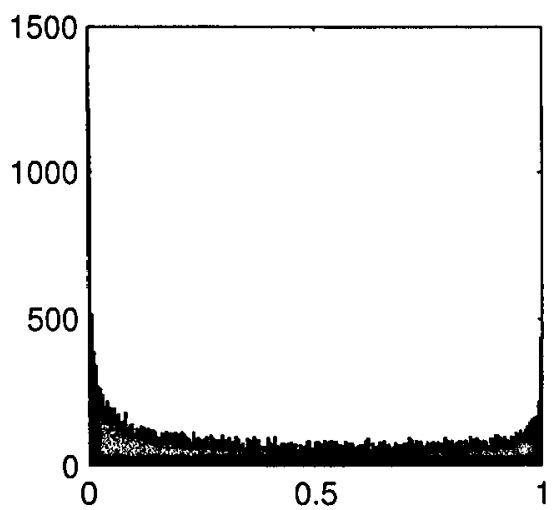

The probability density function $(N=2000)$ averaged from 10 experiments

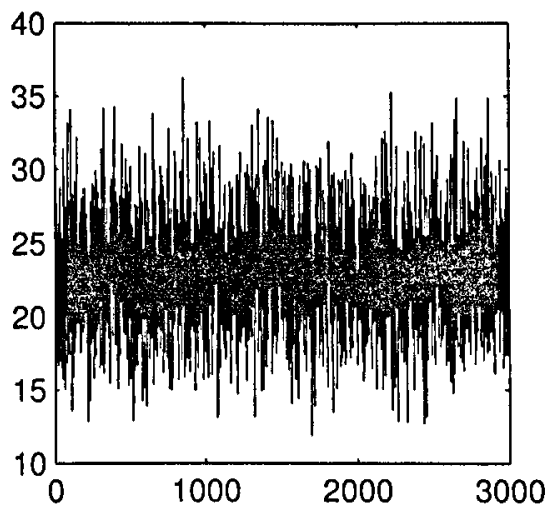

The spectral density Fourier transform of $x_{1}, x_{15}, \ldots, x_{15 N} \quad(N=6000)$ averaged from 10 experiments 
Figure 6.4: Properties of the tangent-feedback map

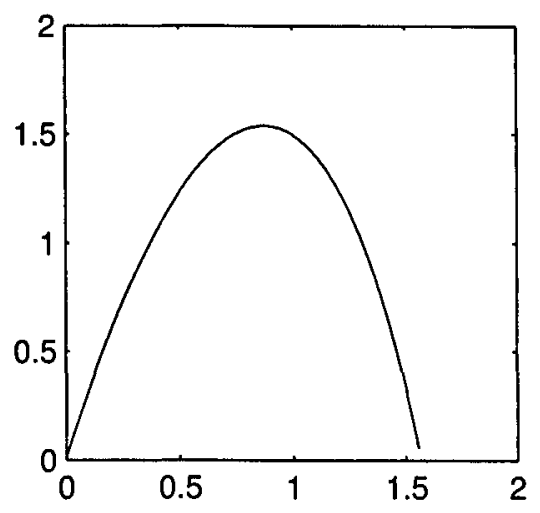

The iterated function $x_{n+1}=f\left(x_{n}\right)$

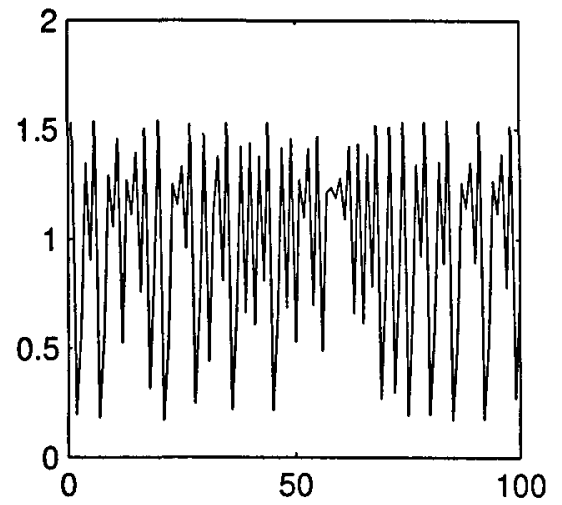

A time series (100 steps) $x_{1}, x_{2}, \ldots, x_{N}$ (shown $N=100$ )

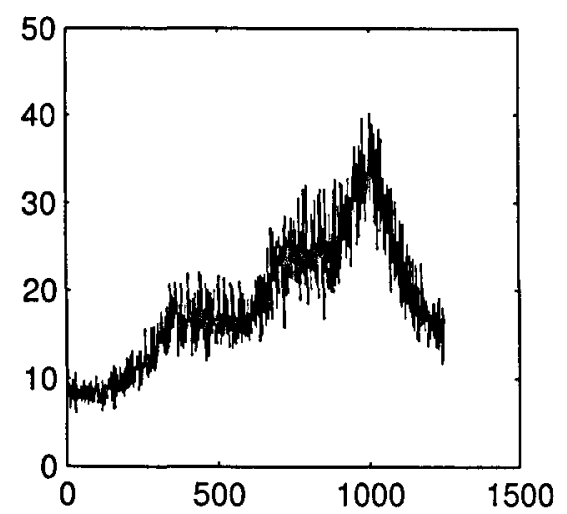

The spectral density Fourier transform of $x_{1}, x_{2}, \ldots, x_{N} \quad(N=6000)$ averaged from 10 experiments

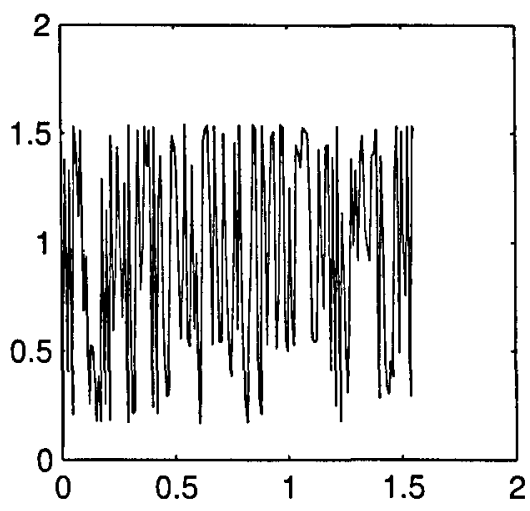

The composition of 15 iterations $x_{n+15}=f^{15}\left(x_{n}\right)$

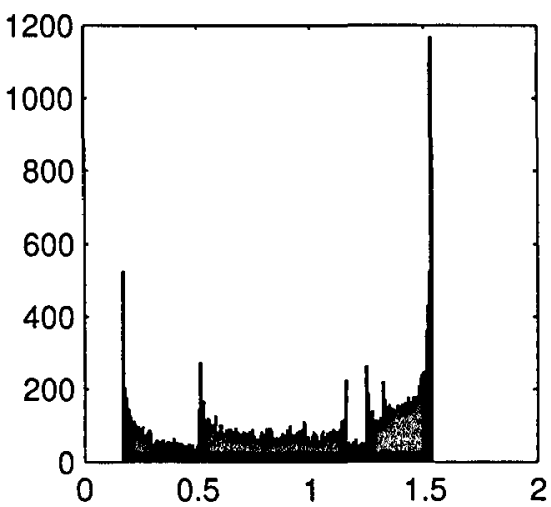

The probability density function $(N=2000)$ averaged from 10 experiments

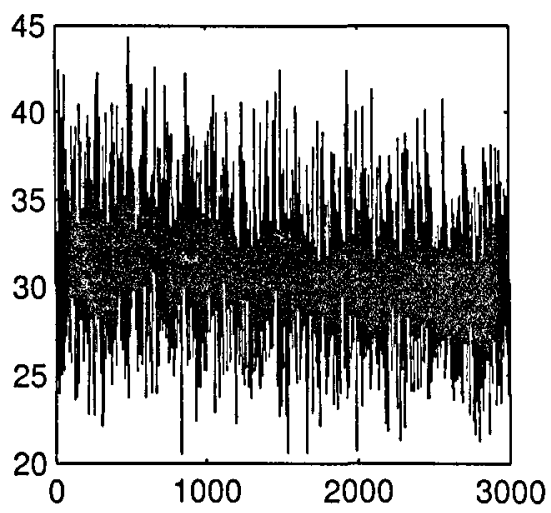

The spectral density Fourier transform of $x_{1}, x_{15}, \ldots, x_{15 N} \quad(N=6000)$ averaged from 10 experiments 
Figure 6.5: Properties of the logarithm-feedback map

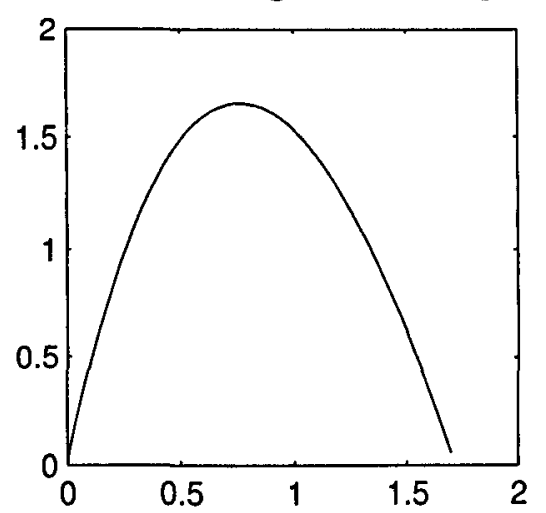

The iterated function $x_{n+1}=f\left(x_{n}\right)$

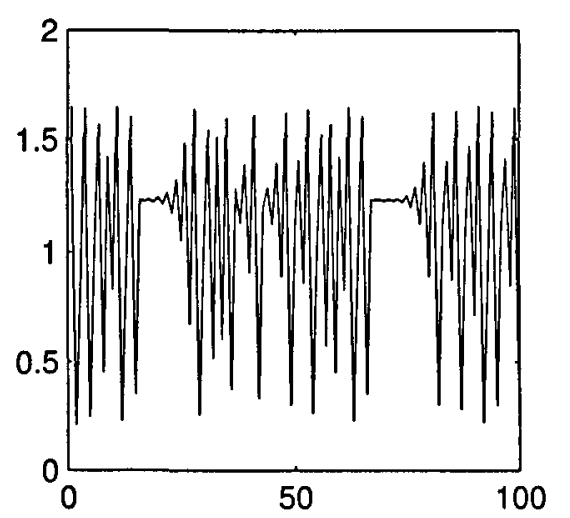

A time series (100 steps) $x_{1}, x_{2}, \ldots, x_{N}$ (shown $N=100$ )

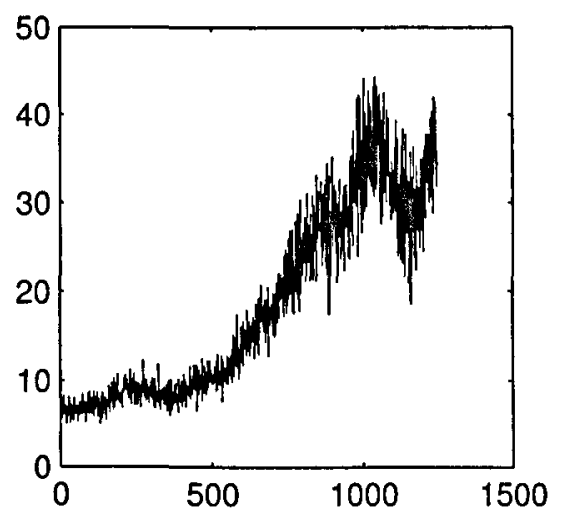

The spectral density Fourier transform of $x_{1}, x_{2}, \ldots, x_{N} \quad(N=6000)$ averaged from 10 experiments

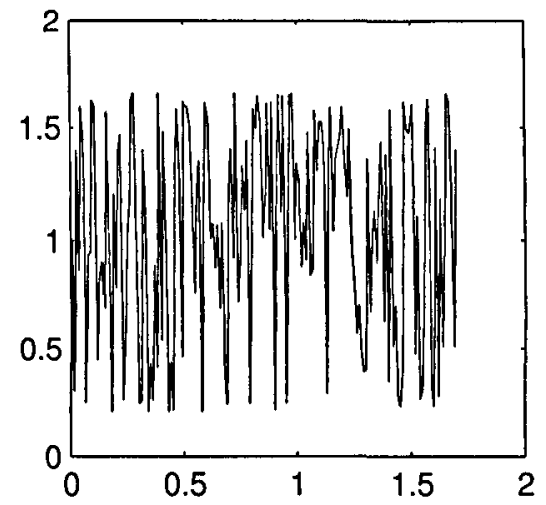

The composition of 15 iterations $x_{n+15}=f^{15}\left(x_{n}\right)$

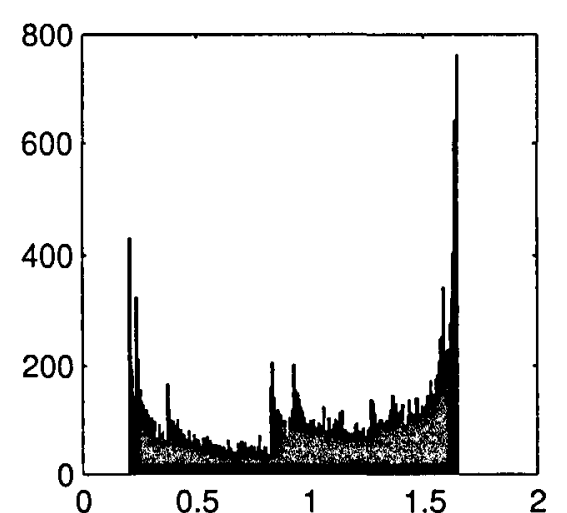

The probability density function $(N=2000)$ averaged from 10 experiments

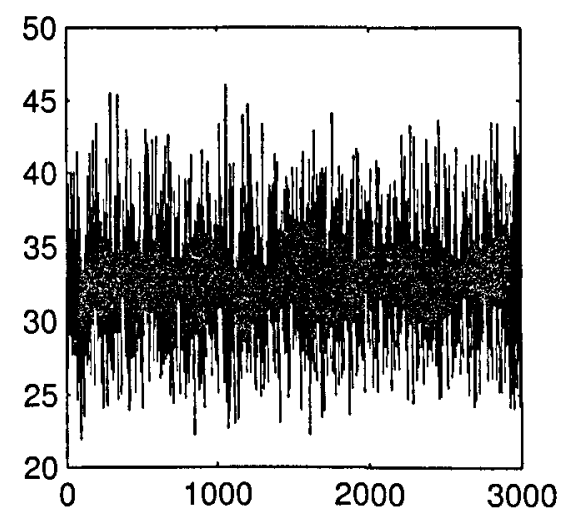

The spectral density Fourier transform of $x_{1}, x_{15}, \ldots, x_{15 N} \quad(N=6000)$ averaged from 10 experiments 
Figure 6.6: Properties of the tent map

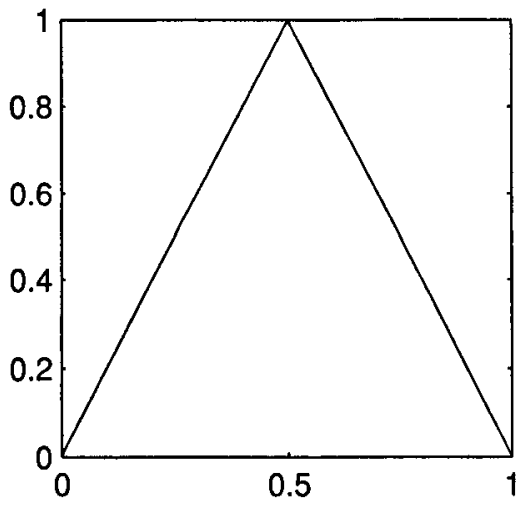

The iterated function $x_{n+1}=f\left(x_{n}\right)$

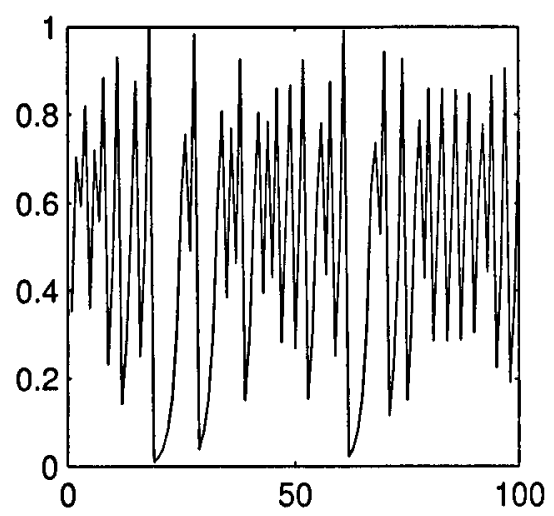

A time series (100 steps) $x_{1}, x_{2}, \ldots, x_{N}$ (shown $N=100$ )

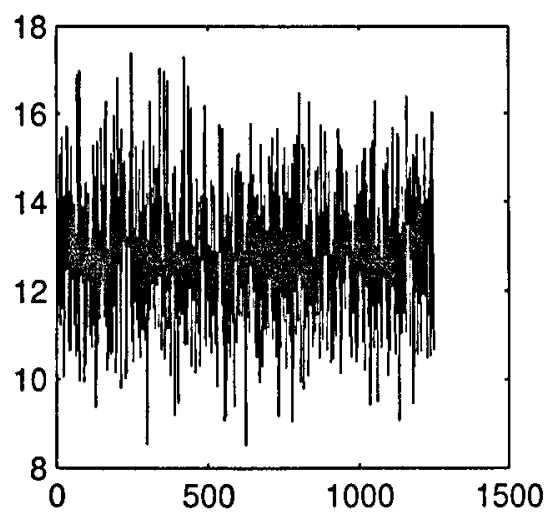

The spectral density Fourier transform of $x_{1}, x_{2}, \ldots, x_{N} \quad(N=6000)$ averaged from 10 experiments

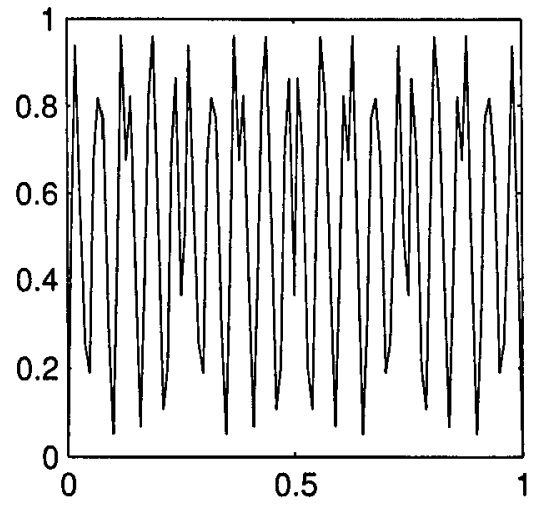

The composition of 15 iterations $x_{n+15}=f^{15}\left(x_{n}\right)$

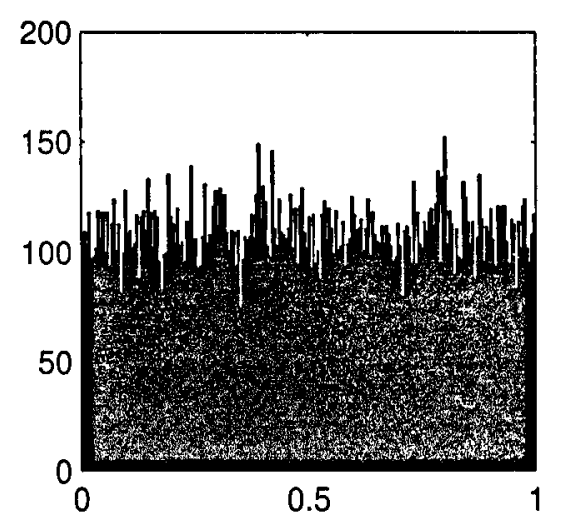

The probability density function $(N=2000)$ averaged from 10 experiments

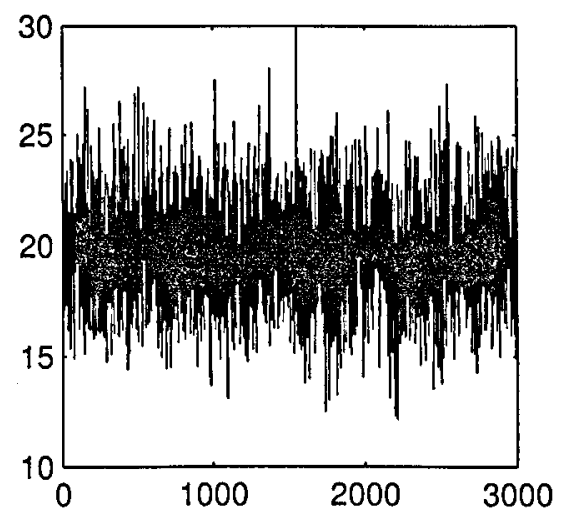

The spectral density Fourier transform of $x_{1}, x_{15}, \ldots, x_{15 N} \quad(N=6000)$ averaged from 10 experiments 
Figure 6.7: Properties of the sawteeth map

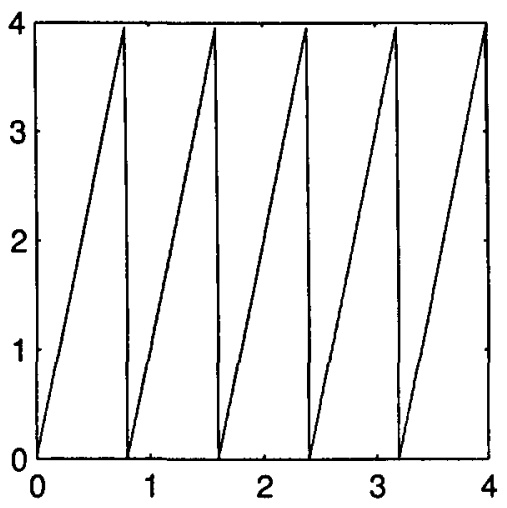

The iterated function $x_{n+1}=f\left(x_{n}\right)$

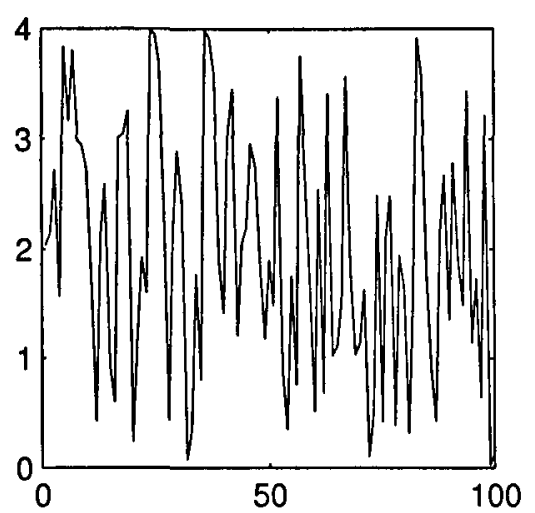

A time series (100 steps) $x_{1}, x_{2}, \ldots, x_{N}$ (shown $N=100$ )

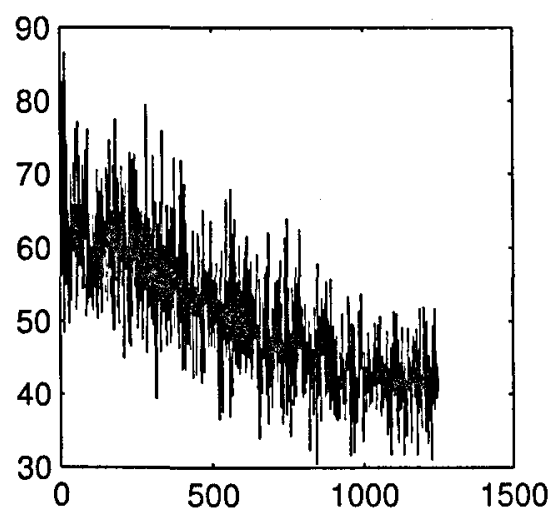

The spectral density Fourier transform of $x_{1}, x_{2}, \ldots, x_{N} \quad(N=6000)$ averaged from 10 experiments

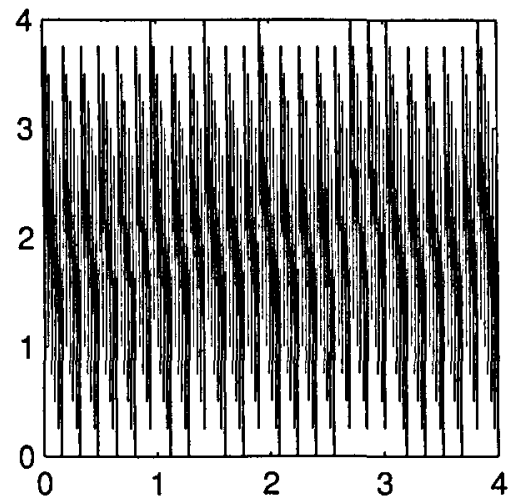

The composition of 15 iterations $x_{n+15}=f^{15}\left(x_{n}\right)$

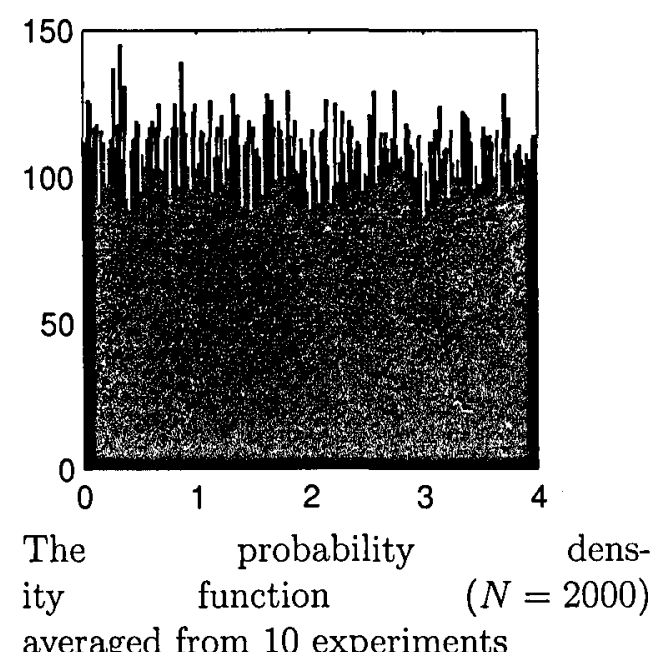

averaged from 10 experiments

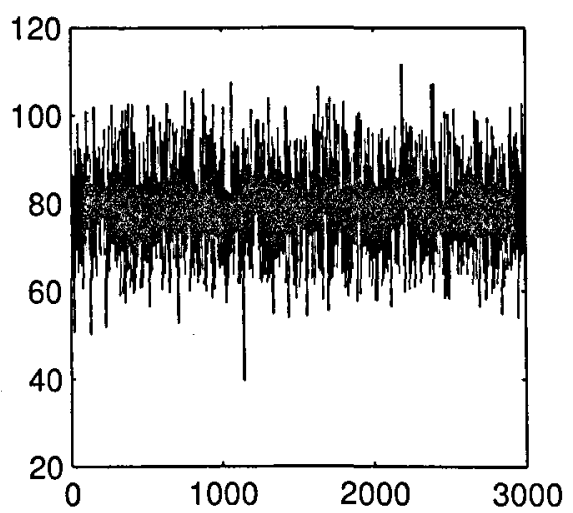

The spectral density Fourier transform of $x_{1}, x_{15}, \ldots, x_{15 N} \quad(N=6000)$ averaged from 10 experiments 
Figure 6.8: Properties of the sawteeth map (cont.)
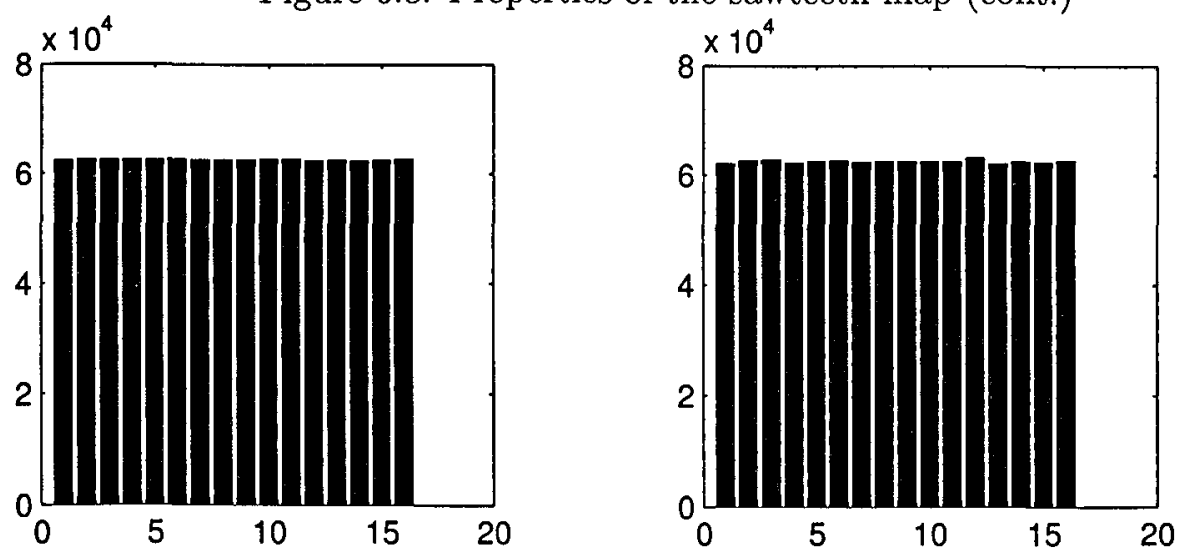

Histogram of 4-bit values obtained us- Histogram of 4-bit values obtained using XOR product of the 2 last signific- ing threshold partitioning at $x_{\text {thres }}=2$ ant bits 
Figure 6.9: Properties of the exactly solvable map

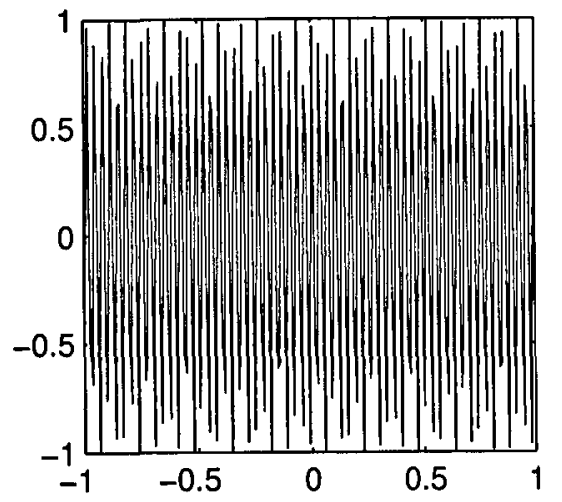

The map $x_{n+1}=f\left(x_{n}\right)$

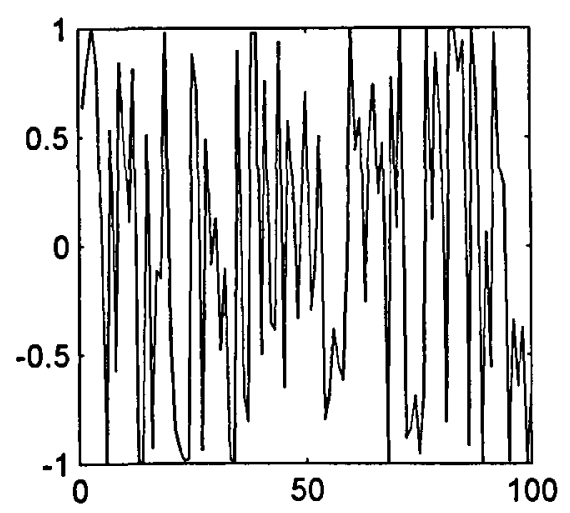

A time series $x_{1}, x_{2}, \ldots, x_{N}$ (shown $N=100$ )

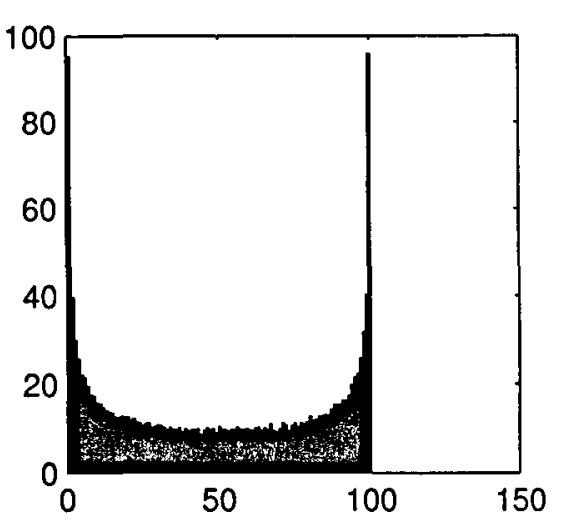

The state distribution

( $N=1000$ first states) averaged from 10 experiments

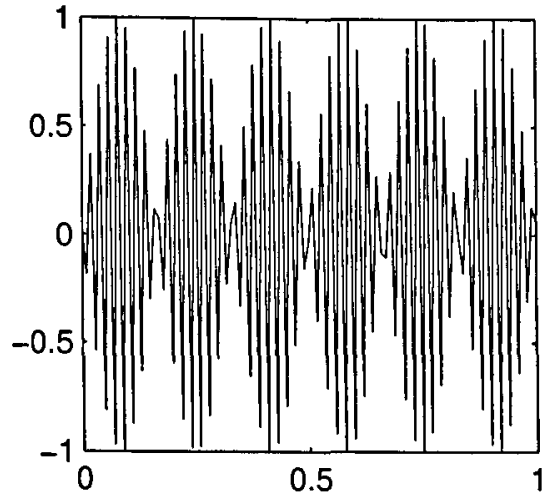

The 15-step map $x_{n+15}=f^{15}\left(x_{n}\right)$

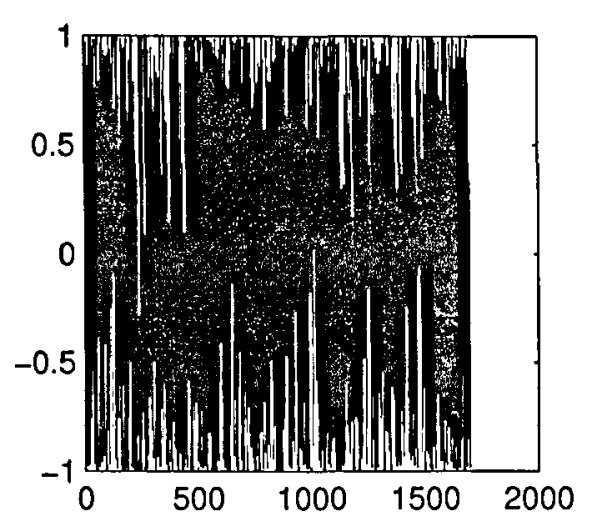

A time series $x_{1}, x_{2}, \ldots, x_{N} \quad(\mathrm{~N}=2000$ steps) After, in average, $N=1860$ the variable $x$ becomes Not-a-Number (NaN).

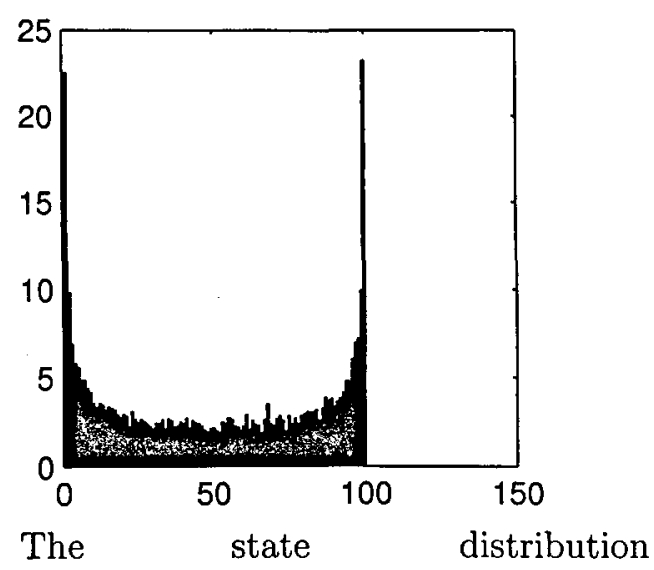

$(N=1000$ last states before $\mathrm{NaN})$ averaged from 10 experiments 
Figure 6.10: Properties of the exactly solvable map (cont.)
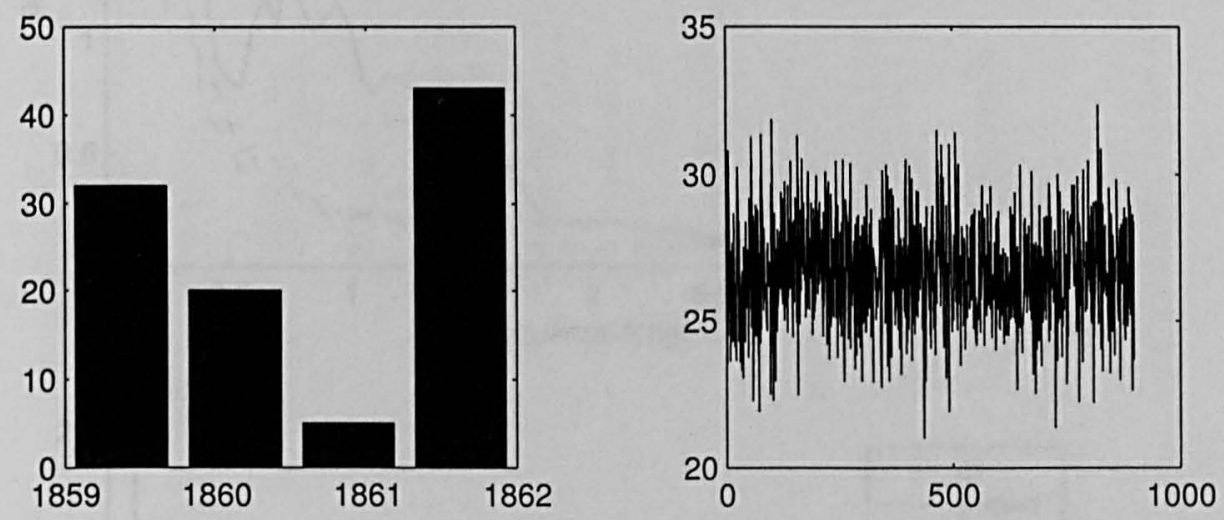

The distribution of cycle lengths The spectral density Fourier trans(averaged from 100 experiments) form of $x_{1}, x_{2}, \ldots, x_{N} \quad(N=1859)$ averaged from 10 experiments 
Figure 6.11: Approximated entropy plots
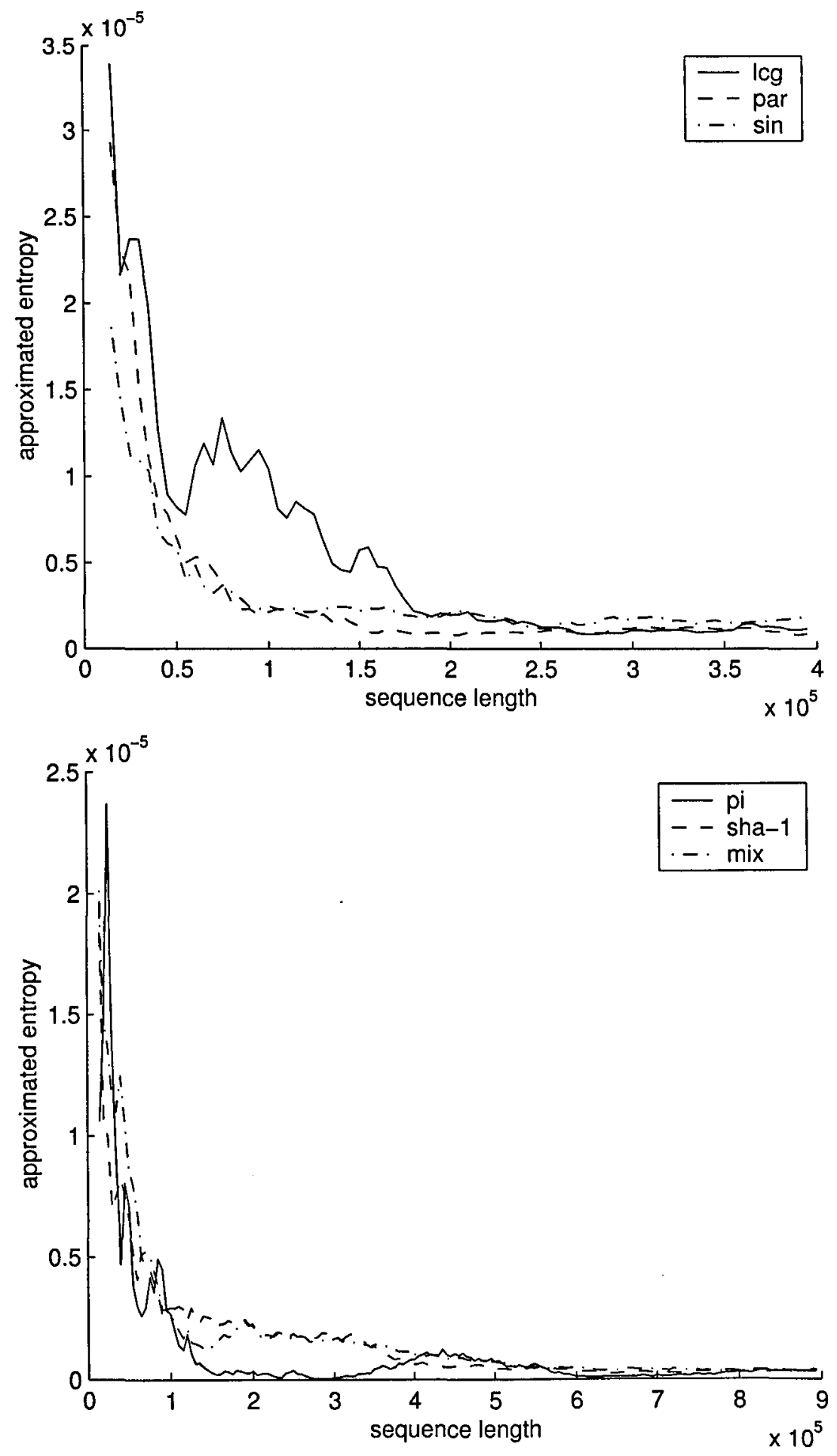
Figure 6.12: Estimation of $P$-value for sha-1, par, par-bad, sin sha-1
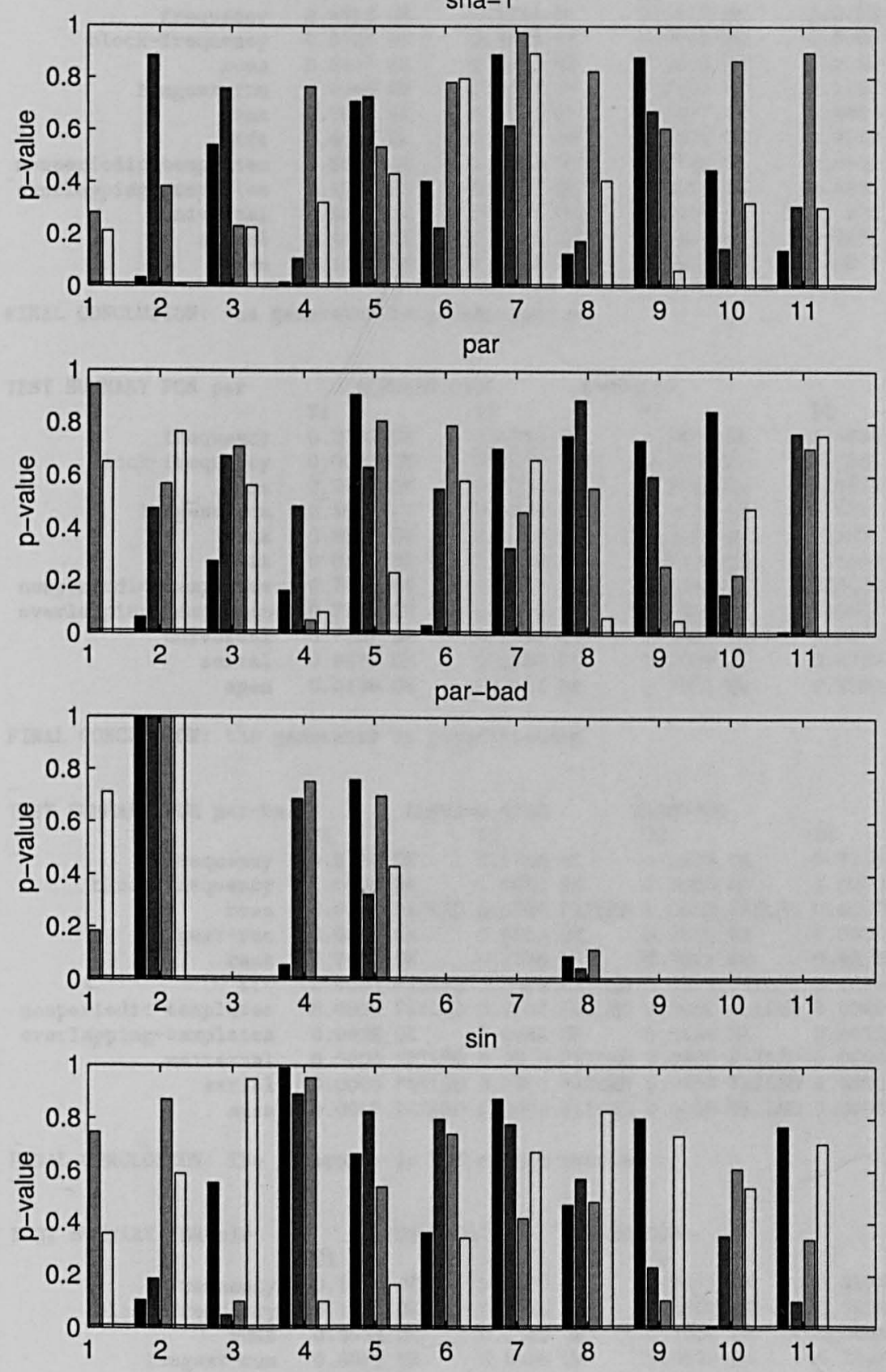
Figure 6.13: Estimation of $P$-value for sha-1, par, par-bad, sin (cont.)

\begin{tabular}{|c|c|c|c|c|c|c|c|}
\hline [EST SUMMARY FOR sha-1 & ALPI & .0100 & $\mathrm{~N}=500000$ & & & & \\
\hline & T1 & T2 & T3 & T4 & & MEAN & CONCL \\
\hline frequency & 0.4955 OK & $0.1414 \mathrm{OK}$ & $0.2812 \mathrm{OK}$ & 0.2123 & $\mathrm{OK}$ & 0.2826 & OK \\
\hline block-frequency & $0.0321 \mathrm{OK}$ & $0.8834 \mathrm{OK}$ & $0.3829 \mathrm{OK}$ & 0.0066 & FAILED & 0.3263 & $\mathrm{OK}$ \\
\hline runs & $0.5417 \mathrm{OK}$ & $0.7580 \mathrm{OK}$ & $0.2309 \mathrm{OK}$ & 0.2258 & $\mathrm{OK}$ & 0.4391 & OK \\
\hline longest-run & $0.0145 \mathrm{OK}$ & $0.1075 \mathrm{OK}$ & $0.7653 \mathrm{OK}$ & 0.3227 & $\mathrm{OK}$ & 0.3025 & $\mathrm{OK}$ \\
\hline rank & $0.70880 \mathrm{~K}$ & $0.7272 \mathrm{OK}$ & $0.5357 \mathrm{OK}$ & 0.4314 & $\mathrm{OK}$ & 0.6008 & $\mathrm{OK}$ \\
\hline fft & $0.4037 \mathrm{OK}$ & $0.2258 \mathrm{OK}$ & $0.7831 \mathrm{OK}$ & 0.7972 & OK & 0.5524 & $\mathrm{OK}$ \\
\hline nonperiodic-templates & $0.8883 \mathrm{OK}$ & $0.6172 \mathrm{OK}$ & $0.9729 \mathrm{OK}$ & 0.8935 & $\mathrm{OK}$ & 0.8430 & $\mathrm{OK}$ \\
\hline overlapping-templates & 0.1275 ox & 0.1777 OK & $0.8251 \mathrm{OK}$ & 0.4092 & OK & 0.3849 & $\mathrm{OK}$ \\
\hline universal & $0.88050 \mathrm{~K}$ & 0.6734 OK & $0.6067 \mathrm{OK}$ & 0.0636 & $\mathrm{OK}$ & 0.5560 & OK \\
\hline serial & $0.4485 \mathrm{OK}$ & $0.14780 \mathrm{~K}$ & $0.8656 \mathrm{OK}$ & 0.3251 & $\mathrm{OK}$ & 0.4467 & $\mathrm{OK}$ \\
\hline apen & $0.1425 \mathrm{OK}$ & $0.3097 \mathrm{OK}$ & $0.8977 \mathrm{OK}$ & 0.3057 & OK & 0.4139 & OK \\
\hline
\end{tabular}

FINAL CONCLUSION: the generator is pseudo-random

\begin{tabular}{rlll} 
TEST SUMMARY FOR par & \multicolumn{3}{c}{ ALPHA=0.0100 } \\
& T1 & $\mathrm{N}=$ \\
frequency & $0.2382 \mathrm{OK}$ & $0.0913 \mathrm{OK}$ \\
block-frequency & $0.0593 \mathrm{OK}$ & $0.4740 \mathrm{OK}$ \\
runs & $0.2758 \mathrm{OK}$ & $0.6723 \mathrm{OK}$ \\
longest-run & $0.1633 \mathrm{OK}$ & $0.4838 \mathrm{OK}$ \\
rank & $0.9078 \mathrm{OK}$ & $0.6305 \mathrm{OK}$ \\
fft & $0.0318 \mathrm{OK}$ & $0.5509 \mathrm{OK}$ \\
nonperiodic-templates & $0.7034 \mathrm{OK}$ & $0.3301 \mathrm{OK}$ \\
overlapping-templates & $0.7518 \mathrm{OK}$ & $0.8890 \mathrm{OK}$ \\
universal & $0.7357 \mathrm{OK}$ & $0.5998 \mathrm{OK}$ \\
serial & $0.8472 \mathrm{OK}$ & $0.1530 \mathrm{OK}$ \\
apen & $0.0138 \mathrm{OK}$ & $0.7627 \mathrm{OK}$
\end{tabular}

FINAL CONCLUSION: the generator is pseudo-random

\begin{tabular}{|c|c|c|c|}
\hline \multicolumn{4}{|l|}{$\mathrm{N}=500000$} \\
\hline T3 & $\mathrm{T} 4$ & MEAN & CONCL \\
\hline $0.9436 \mathrm{OK}$ & $0.6509 \mathrm{OK}$ & 0.4810 & OK \\
\hline $0.5685 \mathrm{OK}$ & $0.7666 \mathrm{OK}$ & 0.4671 & OK \\
\hline $0.7110 \mathrm{OK}$ & $0.5622 \mathrm{OK}$ & 0.5553 & OK \\
\hline $0.0514 \mathrm{OK}$ & $0.0810 \mathrm{OK}$ & 0.1949 & OK \\
\hline $0.8071 \mathrm{OK}$ & $0.2331 \mathrm{OK}$ & 0.6446 & $\mathrm{OK}$ \\
\hline $0.7901 \mathrm{OK}$ & 0.5819 OK & 0.4887 & $\mathrm{OK}$ \\
\hline $0.4643 \mathrm{OK}$ & $0.6623 \mathrm{OK}$ & 0.5400 & $\mathrm{OK}$ \\
\hline $0.5553 \mathrm{OK}$ & $0.0655 \mathrm{OK}$ & 0.5654 & OK \\
\hline $0.2603 \mathrm{OK}$ & $0.0552 \mathrm{OK}$ & 0.4127 & $\mathrm{OK}$ \\
\hline $0.2282 \mathrm{OK}$ & $0.4795 \mathrm{OK}$ & 0.4270 & OK \\
\hline $0.7051 \mathrm{OK}$ & $0.7550 \mathrm{OK}$ & 0.5591 & OK \\
\hline
\end{tabular}

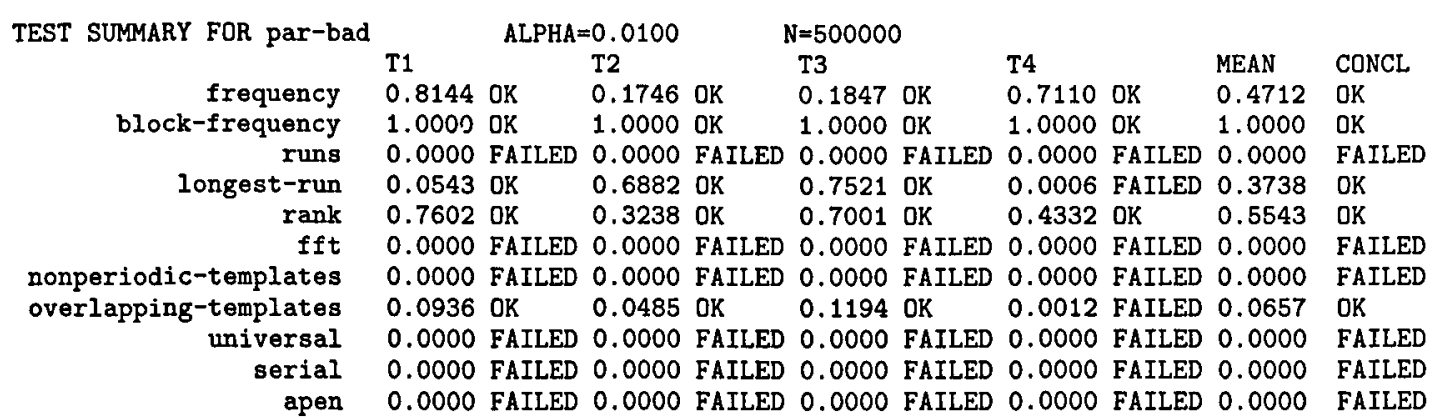

FINAL CONCLUSION: The generator is NOT pseudo-random

\begin{tabular}{|c|c|c|c|c|c|c|c|c|c|c|}
\hline TEST SUMMARY FOR $\sin$ & ALI & $\mathrm{HA}=0.01$ & 100 & & 0000 & & & & & \\
\hline & T1 & & $\mathrm{T} 2$ & & T3 & & T4 & & MEAN & CONCL \\
\hline frequency & 0.1094 & OK & 0.9391 & $\mathrm{OK}$ & 0.7471 & OK & 0.3595 & OK & 0.5388 & OK \\
\hline block-frequency & 0.1032 & OK & 0.1848 & OK & 0.8700 & $\mathrm{OK}$ & 0.5903 & $\mathrm{OK}$ & 0.4371 & OK \\
\hline runs & 0.5558 & $\mathrm{OK}$ & 0.0510 & OK & 0.1009 & $\mathrm{OK}$ & 0.9468 & $\mathrm{OK}$ & 0.4136 & $\mathrm{OK}$ \\
\hline longest-run & 0.9895 & $\mathrm{DK}$ & 0.8905 & OK & 0.9523 & OK & 0.1007 & OK & 0.7332 & $\mathrm{OK}$ \\
\hline rank & 0.6649 & $\mathrm{OK}$ & 0.8253 & $\mathrm{OK}$ & 0.5374 & $\mathrm{OK}$ & 0.1657 & OK & 0.5483 & $\mathrm{OK}$ \\
\hline$f f t$ & 0.3636 & OK & 0.7972 & $\mathrm{OK}$ & 0.7411 & OK & 0.3446 & $\mathrm{OK}$ & 0.5616 & $\mathrm{OK}$ \\
\hline aperiodic-templates & 0.3484 & $\mathrm{OK}$ & 0.8734 & $\mathrm{OK}$ & 0.0687 & OK & 0.1330 & $\mathrm{OK}$ & 0.3559 & OK \\
\hline erlapping-templates & 0.4719 & $\mathrm{OK}$ & 0.5722 & $\mathrm{OK}$ & 0.4818 & OK & 0.8303 & OK & 0.5891 & OK \\
\hline universal & 0.8035 & $\mathrm{OK}$ & 0.2317 & OK & 0.1068 & OK & 0.7345 & $\mathrm{OK}$ & 0.4691 & $\mathrm{OK}$ \\
\hline serial & 0.0050 & FAILED & 0.3522 & $\mathrm{OK}$ & 0.6083 & OK & 0.5354 & $\mathrm{OK}$ & 0.3752 & OK \\
\hline apen & 0.9168 & $\mathrm{OK}$ & 0.1010 & OK & 0.3373 & OK & 0.7041 & $\mathrm{OK}$ & 0.5148 & $\mathrm{OK}$ \\
\hline
\end{tabular}

FINAL CONCLUSION: the generator is pseudo-random 
Figure 6.14: Estimation of $P$-value for tan, log, saw, mix
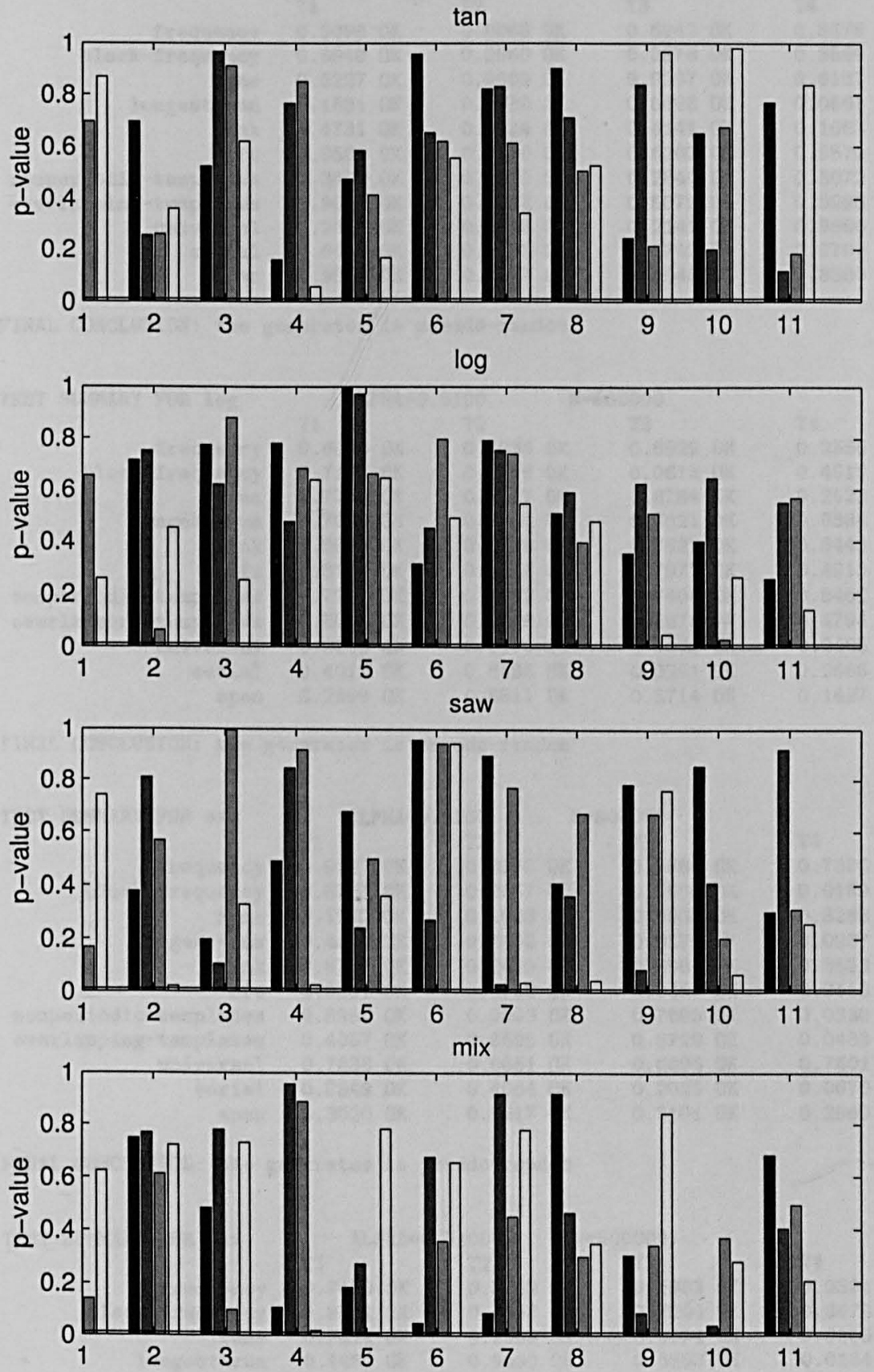
Figure 6.15: Estimation of $P$-value for tan, log,saw,mix (cont.)

\begin{tabular}{rlll} 
TEST SUMMARY FOR tan & \multicolumn{3}{c}{ ALPHA $=0.0100$} \\
frequency & $0.9099 \mathrm{OK}$ & $0.6963 \mathrm{OK}$ \\
block-frequency & $0.6948 \mathrm{OK}$ & $0.2550 \mathrm{OK}$ \\
runs & $0.5227 \mathrm{OK}$ & $0.9660 \mathrm{OK}$ \\
longest-run & $0.1581 \mathrm{OK}$ & $0.7639 \mathrm{OK}$ \\
rank & $0.4731 \mathrm{OK}$ & $0.5834 \mathrm{OK}$ \\
$\mathrm{fft}$ & $0.9561 \mathrm{OK}$ & $0.6530 \mathrm{OK}$ \\
nonperiodic-templates & $0.3499 \mathrm{OK}$ & $0.6699 \mathrm{OK}$ \\
overlapping-templates & $0.9012 \mathrm{OK}$ & $0.7102 \mathrm{OK}$ \\
universal & $0.2447 \mathrm{OK}$ & $0.8365 \mathrm{OK}$ \\
serial & $0.6498 \mathrm{OK}$ & $0.2003 \mathrm{OK}$ \\
apen & $0.9047 \mathrm{OK}$ & $0.1157 \mathrm{OK}$
\end{tabular}

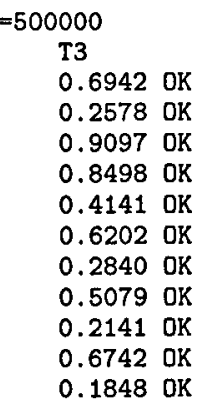

T4

$0.8675 \mathrm{OK}$

0.3586 OK

$0.6187 \mathrm{OK}$

$0.0561 \mathrm{OK}$

0.1683 OK

$0.5570 \mathrm{OK}$

0.3072 OK

$0.5283 \mathrm{OK}$

$0.9650 \mathrm{OK}$

$0.9792 \mathrm{OK}$

$0.8380 \mathrm{OK}$
MEAN

0.3915 OK

0.7543 DK

$0.4570 \quad$ OK

0.4097 OK

$0.6966 \quad \mathrm{OK}$

0.4027 OK

0.6619 OK

0.5651 OK

$0.6259 \quad \mathrm{OK}$

0.5108 OK

FINAL CONCLUSION: the generator is pseudo-random

\begin{tabular}{rlll} 
TEST SUMMARY FOR log & \multicolumn{3}{c}{ ALPHA $=0.0100$} \\
frequency & $0.6028 \mathrm{OK}$ & $0.8034 \mathrm{OK}$ \\
block-frequency & $0.7123 \mathrm{OK}$ & $0.7516 \mathrm{OK}$ \\
runs & $0.7748 \mathrm{OK}$ & $0.6187 \mathrm{OK}$ \\
longest-run & $0.7805 \mathrm{OK}$ & $0.4734 \mathrm{OK}$ \\
rank & $0.9996 \mathrm{OK}$ & $0.9716 \mathrm{OK}$ \\
fft & $0.3128 \mathrm{OK}$ & $0.4518 \mathrm{OK}$ \\
nonperiodic-templates & $0.7937 \mathrm{OK}$ & $0.7542 \mathrm{OK}$ \\
overlapping-templates & $0.5094 \mathrm{OK}$ & $0.5908 \mathrm{OK}$ \\
universal & $0.3550 \mathrm{OK}$ & $0.6270 \mathrm{OK}$ \\
serial & $0.4012 \mathrm{OK}$ & $0.6486 \mathrm{OK}$ \\
apen & $0.2599 \mathrm{OK}$ & $0.5511 \mathrm{OK}$
\end{tabular}

FINAL CONCLUSION: the generator is pseudo-random

$=500000$
$T 3$
$0.6529 \quad 0 K$
$0.0613 \quad 0 K$
$0.8784 \quad 0 K$
$0.6821 \quad 0 K$
$0.6627 \quad 0 K$
$0.7972 \quad 0 K$
$0.7406 \quad 0 K$
$0.3978 \quad 0 K$
$0.5082 \quad 0 K$
$0.0291 \quad 0 K$
$0.5714 \quad 0 K$

$\mathrm{T} 4$

0.2555 OK

0.4519 OK

$0.25280 \mathrm{KK}$

$0.6384 \mathrm{OK}$

$0.6443 \mathrm{OK}$

$0.4913 \mathrm{OK}$

$0.5462 \mathrm{OK}$

$0.4794 \mathrm{OK}$

$0.0485 \mathrm{OK}$

$0.2666 \mathrm{OK}$

$0.1427 \mathrm{OK}$
MEAN

0.5787 OK

0.4943 OK

$0.6312 \mathrm{OK}$

$0.6436 \quad \mathrm{OK}$

$0.8195 \quad \mathrm{OK}$

0.5133 OK

0.7087 OK

0.4944 OK

$0.3847 \quad \mathrm{OK}$

$0.3364 \quad \mathrm{OK}$

0.3813 OK
TEST SUMMARY FOR saw

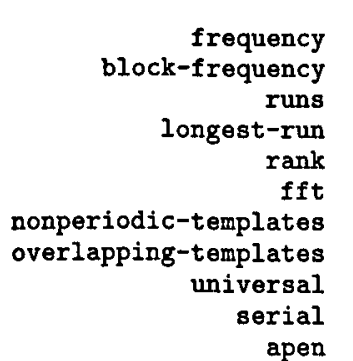

ALPHA $=0.0100$

0.9122 oK

0.3757 OK

0.1952 OK

0.4881 OK

0.6757 OK

$0.9561 \mathrm{OK}$

$0.8957 \mathrm{OK}$

$0.4057 \mathrm{OK}$

$0.7838 \mathrm{OK}$

$0.8549 \mathrm{OK}$

$0.3020 \mathrm{OK}$
0.8875 OK

$0.8057 \mathrm{OK}$

0.1033 OK

$0.8442 \mathrm{DK}$

$0.2403 \mathrm{OK}$

0.2708 OK

$0.0303 \mathrm{OK}$

$0.3595 \mathrm{OK}$

$0.0851 \mathrm{OK}$

$0.4084 \mathrm{DK}$

0.9217 OK
$N=500000$

T3

$0.1684 \mathrm{OK}$

$0.5660 \mathrm{OK}$

0.9933 OK

$0.9170 \mathrm{OK}$

$0.4983 \mathrm{OK}$

$0.9415 \mathrm{OK}$

0.7683 OK

$0.6720 \mathrm{OK}$

0.6696 oK

$0.2025 \mathrm{OK}$

$0.3101 \mathrm{OK}$
T4

$0.7386 \mathrm{OK}$ $0.0189 \mathrm{OK}$

$0.3263 \mathrm{OK}$

$0.0237 \mathrm{OK}$

$0.3593 \mathrm{OK}$

$0.9415 \mathrm{DK}$

$0.0330 \mathrm{DK}$

0.0433 OK

$0.7601 \mathrm{OK}$

$0.0670 \mathrm{OK}$

$0.2560 \mathrm{OK}$
MEAN

0.6767 OK

$0.4416 \quad \mathrm{OK}$

0.4045 OK

$0.5683 \mathrm{OK}$

0.4434 OK

0.7775 OK

$0.4318 \quad \mathrm{OK}$

$0.3701 \mathrm{OK}$

$0.5746 \quad O K$

0.3832 OK

0.4475 OK

FINAL CONCLUSION: the generator is pseudo-random

\begin{tabular}{rlll} 
TEST SUMMARY FOR mix & \multicolumn{3}{c}{ ALPHA $=0.0100$} \\
& $\mathrm{~T} 1$ & $\mathrm{~T} 2$ \\
frequency & $0.7450 \mathrm{OK}$ & $0.3942 \mathrm{OK}$ \\
block-frequency & $0.9304 \mathrm{OK}$ & $0.4553 \mathrm{OK}$ \\
runs & $0.3494 \mathrm{OK}$ & $0.1509 \mathrm{OK}$ \\
longest-run & $0.4450 \mathrm{OK}$ & $0.9430 \mathrm{OK}$ \\
rank & $0.0195 \mathrm{OK}$ & $0.4050 \mathrm{OK}$ \\
fft & $0.6947 \mathrm{OK}$ & $0.0394 \mathrm{OK}$ \\
nonperiodic-templates & $0.2308 \mathrm{OK}$ & $0.8101 \mathrm{OK}$ \\
overlapping-templates & $0.0385 \mathrm{OK}$ & $0.0943 \mathrm{OK}$ \\
universal & $0.9305 \mathrm{OK}$ & $0.0503 \mathrm{OK}$ \\
serial & $0.0954 \mathrm{OK}$ & $0.4085 \mathrm{OK}$ \\
apen & $0.0245 \mathrm{OK}$ & $0.0293 \mathrm{OK}$
\end{tabular}

FINAL CONCLUSION: the generator is pseudo-random
T4

T3

0.1983 OK $0.7304 \mathrm{OK}$ 0.0394 OK $0.6398 \mathrm{OK}$ 0.0902 OK

0.5504 OK $0.0389 \mathrm{OK}$

0.8429 OK

$0.6932 \mathrm{OK}$

$0.7430 \mathrm{OK}$

$0.9045 \mathrm{OK}$
0.0394 OK

$0.8473 \mathrm{OK}$

$0.9650 \mathrm{DK}$

$0.0134 \mathrm{OK}$

$0.2480 \mathrm{OK}$

$0.7430 \mathrm{OK}$

$0.9889 \mathrm{OK}$

$0.4590 \mathrm{OK}$

$0.4305 \mathrm{OK}$

$0.7102 \mathrm{OK}$

$0.0893 \mathrm{OK}$
MEAN

0.3442 OK

0.7408 OK

0.3761 OK

0.5103 OK

0.1907 OK

0.5068 OK

0.5172 OK

0.3586 OK

0.5261 OK

0.4893 OK

0.2619 OK

NCL

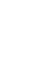


Figure 6.16: Estimation of $P$-value for mix (long sequence)

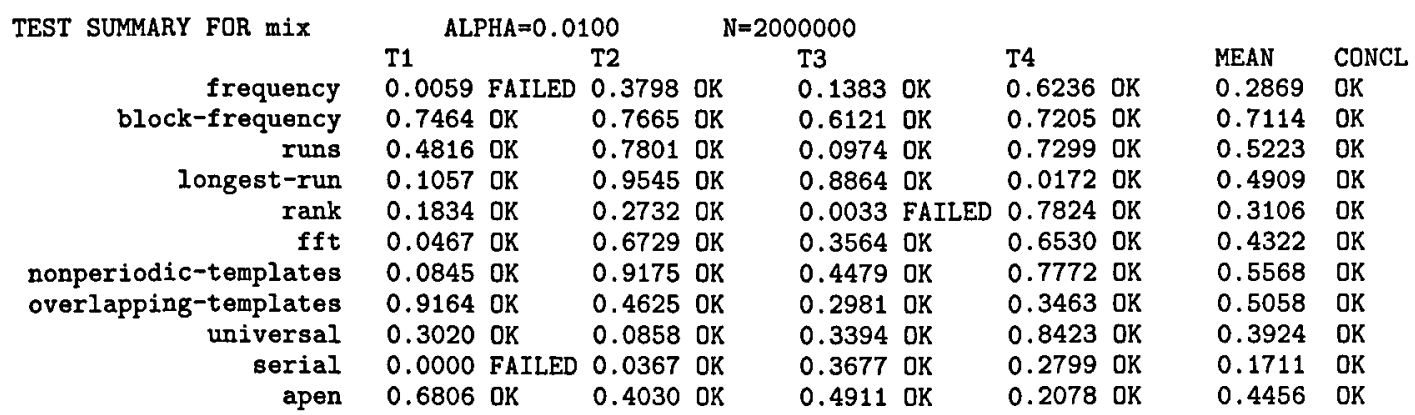

FINAL CONCLUSION: the generator is pseudo-random 


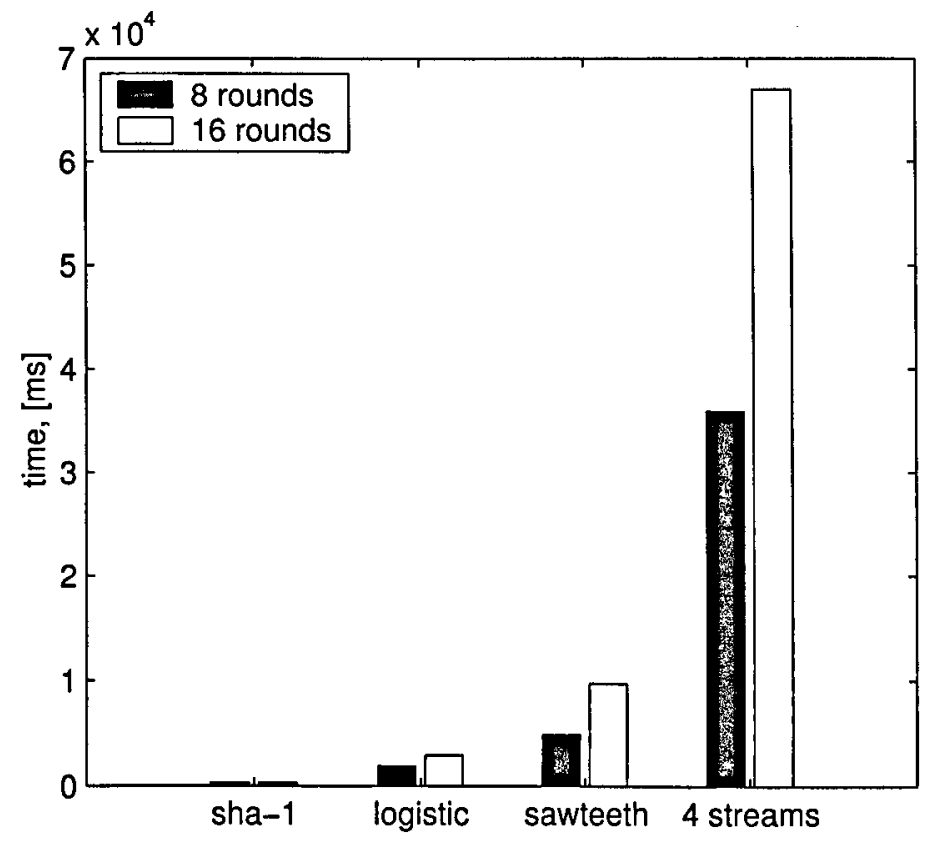

Figure 6.17: Performance evaluation of pseudo-random generators

\subsection{Performance Evaluation}

Figure 6.17 gives run times in milliseconds of different generators: SHA1G, logistic system, sawteeth system and E-Larm (4 streams combo). The test was made with $500 \mathrm{~K}$ sequences in Java Runtime Environment version 1.4 running on Pentium III $1 \mathrm{GHz}$. The time includes the hard-disk output operation. The number of rounds ( 8 or 16) is the number of intermediate iterations between output bit extracting and mixing. Floating-point generators, particulary, E-Larm, are very slow versus a conventional PRNG (SHA1G). Numerically, the E-Larm algorithm is slower than SHA1G by 100-300 fold depending on pseudo-chaotic systems used.

\subsection{Conclusions}

The E-Larm implementation of a pseudo-chaotic generator has passed major statistical tests and, from the null hypothesis, can be considered pseudo-random. Longsequence test $(N=2,000,000)$ for the mix has shown more failures of sequence 
subtests than the basic tests $(N=500,000)$. Clearly, the statistical tests identify more 'non-random' patterns as we increase the length of sequences. It is very likely that the tests will fail if we take a sufficiently long sequence (say, $N \sim 10,000,000$ ).

No theoretical cause was found to confirm the pseudo-random property because of iterative rounding, whose crucial impact was too complex to study analytically. Other disadvantages of E-Larm versus conventional PRNG isolate this approach from modern cryptographic applications. In particular:

- The trajectory length is not perfect and not even stable, i.e. the generator has random cycles, including very short patterns. No mathematical instrument is known to estimate the cycle length and find control parameters to produce perfect trajectories, which is not the case for binary generators.

- Compared with modern PRNG's, the E-Larm implementation is complex and inefficient. It requires floating-point processing, float-to-bit transformation and other unnecessary time-consuming operations. A floating-point format is redundant for the present cryptographic application and the overall performance is not acceptable. 


\section{Chapter 7}

\section{Conclusions}

\subsection{Theory of Chaos based Cryptography}

This thesis has discussed cryptography in the context of chaos theory. Clearly, there is a fundamental relationship between the two sciences. In both chaos theory and cryptography, the object of study is a dynamic system that performs an iterative nonlinear transformation of information in an apparently unpredictable but deterministic manner. In terms of chaos theory, the sensitivity to the initial conditions together the mixing property ensures cryptographic confusion and diffusion, as originally suggested by Shannon.

At the same time, there are conceptual differences:

- Chaos theory studies dynamic systems defined on an infinite state space (e.g vectors of real numbers or infinite binary strings), whereas cryptography relies on a finite-state machine (computer). All chaos models implemented on a computer are approximations, i. e. pseudo-chaos.

- Chaos theory studies the asymptotic behavior of a nonlinear system $(n \rightarrow \infty)$, whereas cryptography focuses on the effect of a small number of iterations $(n \ll \infty)$.

- Chaos theory is not concerned about the algorithmic complexity of the iterated function, while in cryptography complexity is the key issue, meaning security. In other words, the concepts of cryptographic security and efficiency have no 
counterparts in chaos theory. However, certain mathematical problems from chaos theory can be adopted to cryptography.

- Classical chaotic systems have visually recognizable attractors, in which the dimension is fractional. Cryptography attempts to maximize the attractor dimension (to an integer) and to hide any visible structure. Unlike chaos in general, cryptographic systems use all combinations of independent variables to be most unpredictable for an observer.

Chaotic systems are algorithmically random and thus cannot be predicted by a deterministic Turing machine even with infinite power. However, chaotic systems are predictable by a probabilistic Turing machine. Finding probabilistically unpredictable chaotic systems is a central problem for chaos based cryptography.

A rarefied sample $x_{k}, x_{2 k}, \ldots x_{n k}, \ldots$ from a time series $x_{1}, x_{2}, x_{3}, \ldots$, produced by a chaotic and mixing system, is asymptotically independent: for any $n$, elements $x_{(n-1) k}$ and $x_{n k}$ will be more and more independent as $k$ increases. So far, ergodic theory explains a confusion mechanism of cryptographic systems (i.e. the statistical independence between the ciphertext and the plaintext, or between pseudo-random numbers).

Chaotic systems with analytical solutions of the form $x_{n}=\Psi\left(x_{0}, n\right)$ and multivalued map $x_{n+1}=f\left(x_{n}\right)$ can, theoretically, deliver computationally unpredictable (pseudo-random) sequences. The advantage of such a generator is the random access, i.e. any element $x_{n}$ can be computed directly from the initial condition (seed) $x_{0}$. The cryptographic secrecy is kept in the seed and the solution $\Psi\left(x_{0}, n\right)$.

The properties summarized above relate to infinite state systems (true chaos), while cryptographic applications run on finite-state computers. Computer approximations of chaos, called pseudo-chaos do not 'converge' with the original model due to the nature of chaos. Therefore, these theoretical results cannot be directly applied to practical cryptography.

In conclusion, we should admit that the impact of chaos theory is quite doubtful. On the one hand the synergetic approach identifies common laws driving cryptographic systems and iterative nonlinear transformation, exponential instability and other properties. On the other hand, this synergetic knowledge is not enough to 
design strong cryptographic systems. Neither chaos, nor pseudo-chaos can guarantee pseudo-randomness and resistance to different kinds of cryptanalyses. Chaos theory is not related to number theory and the computational complexity that underpin digital cryptography.

For many years, symmetric ciphers and PRNG's have been deeply studied and do not seem to need serious upgrades. By contrast, asymmetric schemes, which modern security relies upon, require much more attention; they form the basis for such crucial applications as key management and digital signature. However, chaos theory, at least in this project, has not brought any new ideas to asymmetric cryptography.

\subsection{Practical Issues}

Floating-point arithmetic is the most obvious solution of approximating continuous chaos on a finite-state machine. However, there is no straightforward application to pseudo-random number generation and ciphering. Critical problems are (i) growing rounding-off errors; (ii) the approximated system is not structurally stable, i.e. different initial conditions and parameters yield different cryptographic properties, such as very short orbits, weak plaintexts or weak keys. These problems have been solved in the E-Larm system software (Chapter 5), but in some (insufficient) degree only.

Chaotic systems based on smooth nonlinear functions (e.g. $x^{2}, \sin (x), \log (x)$ ) produce sequences with a highly non-uniform distribution. Clearly, these can be predicted by a probabilistic machine. Using the last significant bit (LSB) partitioning and multi-round transformation, we obtain a bit sequence with good statistical properties. It passes all pseudo-randomness tests, from which we conclude that it is pseudo-random.

Many piecewise-linear maps (e.g. sawteeth, tent map) generate sequences, which have theoretically flat distributions. But in practice, these are even more dangerous than nonlinear systems because of the overall effect of linearity, rounding and iterative transformations. Often, histograms of bit sequences produced by piecewise-linear maps, look like a staircase or a large saw. The LSB method does not work (last bits display patterns), instead, the output can be produced from first significant bit(s) 
or using a threshold.

As shown in Chapter 6, floating-point chaos based cryptosystems, both nonlinear and piecewise-linear are dramatically inefficient when compared to conventional PRNG's. Other shortcomings, particulary, the lack of theoretical conviction in their pseudo-random property make them unacceptable to modern cryptography.

All conventional cryptographic systems (encryption schemes, pseudo-random generators, hash functions) are binary pseudo-chaotic systems, defined on a finite space of strings. Such systems are periodical but have a limited sensitivity to the initial conditions, i.e. the Lyapunov exponents are positive only if measured at the beginning of the process (before one can see the cycles). The mixing property leads to pseudo-randomness.

Pseudo-chaotic systems have typically many orbits of different length. Measuring the minimal, average and maximal length of a system is not a trivial number theory problem. Clearly, ideal cryptographic systems have a single orbit that includes all the possible states.

Iterative block ciphers can be viewed as a combination of two linked pseudochaotic systems: data and round-key systems. The iterated function of the data system includes: nonlinear substitutions, row shifts, column mixing etc. The roundkey system is a pseudo-random generator providing the sensitive dependence of the ciphertext on the key.

Technically, most of pseudo-random generators are based on the stretch-and-fold transformation: first, the state is stretched over a large space (e.g, multiplying or raising in power), then folded into the original state space (using a periodical function such as mod, sin). In mathematical chaos, the stretch-and-fold transformation forms the basis of the majority of iterated functions.

An exact (analytical) solution of a chaotic system has a much stronger stretchand-fold property. Consequently, it eliminates the precision problem in some degree only: in the beginning, the simulation of chaos is more accurate, but, for long sequences the result is even worse than iterative computations.

Floating-point implementations of analytical solutions do not provide good pseudorandom properties. In particular, orbits can be unpredictably short and their length depends on the initial condition(s). 
Another problem is that we know analytical solutions only for certain (boundary) values of the control parameters. This does not allow the use of parameters as a secret key; all the secrecy must be kept in the seed.

\subsection{Future Work}

As discussed above, chaotic dynamics, which essentially describes real (continuous) state systems, does not really contribute to digital cryptography. However, the following future directions can be suggested with respect to binary chaos:

Structurally stable pseudo-chaotic systems It is very important to have a structurally stable cryptosystem, i.e. a system that has (almost) the same cycle length and Lyapunov exponents for all initial conditions and control parameters. Most of the known pseudo-chaotic systems do not possess this quality.

Increasing-precision pseudo-chaotic systems All approximations of chaos studied within this work are based on fixed precision computations. However, it is possible to increase the precision or resolution (e.g. the length of a binary state string) in each iteration. Furthermore, the precision can vary up and down according to a set of rules, defined to estimate the error impact. This technique would be very important in simulation sciences.

Conditions of unpredictability for chaotic systems It is still an open question as to what properties of a chaotic system guarantee its computational unpredictability. Known unpredictable systems have emerged from number theory problems. It is possibly that this is the only way to find unpredictable systems.

Invertible pseudo-chaotic systems A one-way transformation forms the basis of a PRNG, whereas a key-dependent invertible transformation is the essence of a cipher or encryption scheme. Most chaos based ciphers extended their chaotic PRNG with invertible transformation (XOR, cyclic shifts and other permutations). The latter transformations can also be considered as pseudochaotic maps. 
Chaos based asymmetric encryption Modern cryptography is based on asymmetric cryptographic systems, based on trapdoor functions, i.e. functions that have the one-way property unless a secret parameter (trapdoor) is known. No counterpart of a trapdoor transformation is known in chaos theory. 


\section{Bibliography}

[1] С. П. Капица, С. П. Курдюмов, and Малинецкий Г. Г. Синергетика и проәнозы будущего. Едиториал УРСC, 2001. // S. P. Kapitsa, S. P. Kurdumov, G. G. Malinensky, Synergetics and forecasts.

[2] Ю. А. Данилов and Б. Б. Кадомцев. Нелинейнъе волны. Самоорганизаuия. Наука, Москва, 1983. http://www.synergetic.ru/science/index. php?article=dan // U. A. Danilov, B. B. Kadomtsev, Nonlinear waves. Selforganization.

[3] М. В. Капранов and В. Г. Чернобаев. Управляемые генераторы хаотических колебаний на базе систем фазовой синхронизации. Радиотехническue mempadu, (15), 1998. // M. V. Kapranov, V. G. Chernobaev, Controlled chaos generators based on phase syncronization systems.

[4] Г. Г. Малинецкий and А. Б. Потапов. Современные проблемы нелинейной динамики. Едиториал УРСC, 2000. // G. G. Malinensky, A. B. Potapov, Modern problems in nonlinear dynamics.

[5] М. Гэри and Д. Джонсон. Вычислительные маиинь и трудно решаемые задачи. Мир, Москва, 1982. // M. R. Garey, D. S. Johnson, Computers and intractability.

[6] Г. Николис and И. Пригожин. Познание сложного. Введение. Мир, 1990. // G. Nicolis, and I. Prigogine, Exploring complexity. An introduction, W. H. Freeman and Company, New York.

[7] А. Дмитриев and С. Старков. Новые подходы к решению проблем в системах связи и компьютерных сетях: динамический хаос. Компътотерра, (46), 
2001. // A. Dmitriev, Novel solutions in comunication system and computer networks: deterministic chaos.

[8] П. Р. Халмош. Теория меры. ИЛ, 1953. // Р. R. Halmos, Measure theory.

[9] Я. Г. Синай. Современные проблемы эргодической теории. ФИЗМАТЛИТ, Москва, 1995. // Y. G. Sinai, Modern problems in ergodic theory.

[10] В. С. Анищенко. Детерминированный хаос. Соросовский образовательный журнал, (6):70-76, 1997. http://www.nsu.ru/materials/ssl/text/ metodics/anishenko.html // V. S. Anishenko, Deterministic Chaos.

[11] Дж. Д. Биркгоф. Динамические системъ. РХД, Ижевск, 1999. // G. D. Birkhoff, Dynamical Systems.

[12] П. Р. Халмош. Лекции по эргодической теории. РХД, Ижевск, 1999. // P. R. Halmos, Lectures on ergodic theory.

[13] В. В. Ященко, editor. Введение в криптографию. МЦНМО, Москва, 2000. // V. V. Yanchenko, Introduction to cryptography.

[14] В. В. Суриков. О термине синергетика. In Синергетика. Трудь семинаpa., volume 3, Москва, 2000. Изд-во МГУ. // V. V. Surikov, On the term synergetics.

[15] А. Ю. Лоскутов. Синергетика и нелинейная динамика: новые подходы к старым проблемам. In Синергетика. Труды семинара., volume 3, Москва, 2000. Изд-во МГУ. // А. Ү. Loskutov, Synergetics and nonlinear dynamics: new approaches to old problems.

[16] Г. Шустер. Детерминированный хаос. Введение. Мир, Москва, 1988. // H. G. Schuster, Deterministic chaos: an introduction, VCH, Weinheim, 1988.

[17] А. Лоскутов. Нелинейная динамика, теория динамического хаоса и синергетика (перспективы и приложения). Компъютерра, (47), 1998. http://www.cplire.ru/win/InformChaosLab/chaoscomputerra/ 
Loskutov.htmI // A. Loskutov, Nonlinear dynamics, chaos theory and synergetics (perspectives and applications).

[18] И. Пригожин. Конеч определенности. Времл, хаос и новые законы. РХД, Ижевск, 1999. // I. Prigogine, The end of certainty. Time, chaos and the new laws of nature, The Free Press, New York, 1997.

[19] А. Дмитриев. Детерминированный хаос и информационные технологии. Наука и жизнь, (5), 2001. http://nauka.relis.ru/cgi/nauka.pl?07+ 0105+07105044+HTML // A. Dmitriev, Deterministic chaos and information technologies.

[20] М. Шредер. Фракталы, хаос, степенные законы. Миниатюры из бесконечного рал. РХД, Ижевск, 2001. // M. Schroeder, Fractals, Chaos, Power Laws. Mintutes from an infinite paradise, W. H. Freeman and Company, New York.

[21] А. Н. Колмогоров. Теория информации и теория алгоритмов. Наука, Москва, 1987. // A. N. Kolmogorov, Information theory and algorithm theory.

[22] M. S. Baptista. Cryptography with chaos. Physics Letters A, 240(1-2):50-54, 1998.

[23] F. L. Bauer. Decrypted Secrets. Springer, 1997.

[24] E. Beham. Cryptanalysis of the chaotic-map cryptosystem. In Proc. of EUROCRYPT'91, 1991. http://citeseer.nj.nec.com/175190.html.

[25] M. E. Bianco and D. Reed. An encryption system based on chaos theory. US Patent No. 5048086, 1991.

[26] J. M. Blackledge. E-larm: super security for e-banking through the application of iterated function systems and fractal modulation for digital encryption. In Proc. IMA Conference on Fractal Geometry: Mathematical Techniques, Algorithms and Applications, 2000. not published.

[27] M. Blum and S. Micali. How to generate crytographically strong sequences of pseudo-random bits. SIAM Journal of Computations, 13(4):850-864, 1984. 
[28] G. Boffetta, M. Cencini, M. Falcioni, and A. Vulpiani. Predictability: a way to characterize complexity, 2001. http://www. unifr.ch/econophysics/.

[29] R. Brown and L. O. Chua. Clarifying chaos: examples and counterexamples. $I J B C, 6(2): 219-249,1996$.

[30] A. A. Brudno. Entropy and the complexity of the trajectories of a dynamical system. Trans. Moscow Mathematical Society, 44, 1983.

[31] A. B. Cambel. Applied Chaos Theory. A Paradigm for Complexity. Academic Press Inc., New-York, 1993.

[32] L. Cappelletti. An FPGA implementation of a chaotic encryption algorithm. Master's thesis, Universita Degli Studi di Padova, 2000. http://www. lcappelletti.f2s.com/Didattica/thesis.pdf.

[33] J. M. Carroll, J. Verhagen, and P. T. Wong. Chaos in cryptography: the escape from the strange attractor. Cryptologia, 16(1):52-72, 1992.

[34] B. V. Chirikov and F. Vivaldi. An algorithmic view of pseudorandomness. Physica D, (129), 1999.

[35] Y. H. Chu and S. Chang. Dynamic cryptography based on synchronized chaotic systems. Electronic Letters, 35(12), 1999.

[36] Y. H. Chu and S. Chang. Dynamic data encryption system based on synchronized chaotic systems. Electronic Letters, 35(4), 1999.

[37] F. Dachselt, K. Kelber, and W. Schwarz. Chaotic coding and cryptoanalysis. http://citeseer.nj.nec.com/355232.html.

[38] F. Dachselt, K. Kelber, J. Vandewalle, and W. Schwarz. Chaotic versus classical stream ciphers - a comparative study, 1998. citeseer.nj.nec.com/ article/dachselt98chaotic.html.

[39] Whitfield Diffie and Martin Hellman. New directions in cryptography. 2(6):644-654, 1976. 
[40] W. Ebeling, L. Molgedey, J. Kurths, and U. Schwarz. Entropy, complexity, predictability and data analysis of time series and letter sequences, 1999. http: //citeseer.nj.nec.com/395066.html.

[41] T. Erber, T. Rynne, W. Darsow, and M. Frank. The simulation of random processes on digital computers: unavoidable order. Journal of Computational Physics, (49):349-419, 1983.

[42] M. J. Feigenbaum. The universal metric properties of nonlinear transformations. J. Stat. Physics, (21):669-706, 1979.

[43] A. Fog. Chaotic random number generators, 1999. http://www.agner.org/ random/theory/.

[44] J. Fridrich. Discrete-time dynamical systems under observational uncertainty. J. Appl. Math. Comp., (83):181-207, 1993.

[45] J. Fridrich. Symmetric ciphers based on two dimension chaotic map. IJBC, 8(6):1259-1284, 1998.

[46] J. Fridrich and J. Geer. Reconstruction of chaotic orbits under finite resolution. J. Appl. Math. Comp., (80):129-159, 1995.

[47] R. Buckminster Fuller and E. J. Applewhite. Synergetics. Explorations in the Geometry of Thinking. Macmillan Publishing, New York, 1979.

[48] P. Gacs. Lecture notes on descriptional complexity and randomness. Computer Science Department, Boston University, 2001. http://cs-pub.bu.edu/ faculty/gacs/Home.html.

[49] J. B. Gallagher and J. Goldstein. Sensitive dependence cryptography, 1996. http://www. navigo.com/sdc/.

[50] O. Goldreich. Introduction to complexity theory. Lecture Note, Department of Computer Science and Applied Mathematics, Weizmann Institute of Science, Israel, 1999. 
[51] J. A. González and R. Pino. Chaotic and stochastic functions. Physica, 276A:425-440, 2000.

[52] H. Gutowitz. Cryptography with dynamical systems. ESPCI, Laboratoire d'Electronique, Paris, France, 1995. http://www.santafe.edu/ hag/ crypto/crypto.html.

[53] T. Habutsu, Y. Nishio, I. Sasase, and S. Mori. A secret key cryptosystem by iterating chaotic map. In Proc. of EUROCRYPT'91, pages 127-140, 1991. http://link.springer.de/link/service/series/0558/bibs/0547/ $05470127 . \mathrm{htm}$.

[54] H. Haken. Synergetics, an introduction: nonequilibrium phase transitions and self-organization in physics, chemistry, and biology, 3rd rev. Springer-Verlag, New York, 1983.

[55] B.-L. Hao. Elementary symbolic dynamics and chaos in dissipative systems. World Scientific Pub Co, 1989.

[56] J. Hastad. Pseudo-random generators under uniform assumptions. In Proceedings 22nd Annu. ACM Symp. on Theory of Computing, pages 385404, 1990.

[57] M. K. Ho. Chaotic encryption techniques. Master's thesis, Department of Electronic Engineering, City University of Hong Kong, 2001. http: //personal.cityu. edu.hk/ $\sim 50115849 /$ ces/.

[58] S. Hollasch. IEEE standard 754: floating point numbers, 1998. http:// research.microsoft.com/ hollasch/cgindex/coding/ieeefloat.html.

[59] J. Hosack. The use of Chebysev mixing to generate pseudo-random numbers. Journal of Computational Physics, (67):482-486, 1986.

[60] R. Impagliazzo, L. Levin, and M. Luby. Pseudo-random generation from oneway functions. In Proc. 21st Annu. ACM Symp. on Theory of Computing, pages 230-235, 1989. 
[61] E. A. Jackson. Perspectives in nonlinear dynamics. Cambridge University Press, 1991.

[62] S. Katsura and W. Fukuda. Exactly solvable models showing chaotic bahavior. Physica, (130A):597-605, 1985.

[63] M. P. Kennedy, R. Rovatti, and G. Setti. Chaotic electronics in telecommunications. CRC Press, 2001.

[64] D. Knuth. The art of computer programming - seminumerical algorithms, volume 2. 2nd ed. Addison-Wesley: Reading, Massachusetts, 1981.

[65] L. Kocarev. Chaos and cryptography, 2001. http://rfic.ucsd.edu/chaos/ ws2001/kocarev.pdf.

[66] L. Kocarev. Chaos-based cryptography: a brief overview. Circuits and systems, $1(3): 6-21,2001$.

[67] L. J. Kocarev, K. S. Halle, K. Eckert, and L. O. Chua. Experimental demonstration of secure communications via chaotic synchronization. IJBC, 2(3):709-713, 1992.

[68] Z. Kotulski, J. Szczepańs, K. Gyrski, A. Gyrska, and A. Paszkiewicz. On constructive approach to chaotic pseudorandom number generators. In Proc. of the Regional Conference on Military Communication and Information Systems, volume 1, pages 191-203. CIS Solutions for an Enlarged NATO, RCMIS, 2000. http://www.ippt.gov.pl/ zkotulsk/CPRBG.pdf.

[69] Z. Kotulski and J. Szczepański. Discrete chaotic cryptography. new method for secure communication. In Proc. NEEDS'97, 1997. http://www . ippt.gov . $\mathrm{pl} /$ zkotulsk/kreta.pdf.

[70] Z. Kotulski and J. Zczepanski. On the application of discrete chaotic dynamical systems to cryptography. DCC method. Biuletyn Wat Rok, XLVIII(10):111123, 1999. http://www.ippt.gov.pl/ zkotulsk/wat.pdf.

[71] D. Lai, G. Chen, and M. Hasler. Distribution of the Lyapunov exponent of the chaotic skew tent map. IJBC, 9(10):2059-2067, 1999. 
[72] L. A. Levin. One-way function and pseudorandom generators. Combinatorica, $7(5): 357-363,1987$.

[73] L. Lovász. Computation complexity. Lecture Notes. http://ftp.cs.yale. edu/pub/lovasz.pub/.

[74] George Marsaglia. The marsaglia random number cdrom including the diehard battery of tests of randomness, 1995. http://stat.fsu.edu/pub/diehard/.

[75] N. Masuda and K. Aihara. Finite state chaotic encryption system, 2000. http://www . aihara.co.jp/rdteam/fs-ces/.

[76] R. Matthews. On the derivation of a chaotic encryption algorithm. Cryptologia, (13):29-42, 1989.

[77] A. J. Menezes, P. C. van Oorschot, and S. A. Vanstone. Handbook of applied cryptology. CRC Press, 1996. http://www.cacr.math.uwaterloo.ca/hac/.

[78] V. I. Oseledec. A multiplicative ergodic theorem: Lyapunov charcteristic numbers for dynamical systems. Trans. Mosc. Math. Soc., 19(197), 1968.

[79] E. Ott, editor. Chaos in Dynamical Systems. Cambridge University Press, 1993.

[80] N. Paar. Robust encryption of data by using nonlinear systems, 1999. http: //www.physik.tu-muenchen.de/ npaar/encript.html.

[81] H.-O. Peitgen, H. Jürgens, and D. Saupe. Chans and fractals. New frontiers of science. Springer, 1992.

[82] V. A. Protopopescu, R. T. Santoro, and J. S. Tolliver. Fast and secure encryption-decryption method. US Patent No. 5479513, 1995.

[83] Nikolai V. Ptitsyn, Jonathan M. Blackledge, and Valery M. Chernenkiy. Deterministic chaos in digital cryptography. In Proceedings of IMA Conference on Fractal Geometry. Horwood Publishing, 2002.

[84] V. Rijmen and J. Daemen. Rijndael algorithm specification, 1999. http: //www.esat.kuleuven.ac.be/ rijmen/rijndael/. 
[85] T. Ritter. Ciphers by ritter, 1991. http://www.ciphersbyritter.com/.

[86] T. Ritter. The efficient generation of cryptographic confusion sequences. Cryptologia, (15):81-139, 1991. http://www.ciphersbyritter.com/ARTS/ CRNG2ART . HTM.

[87] R. L. Rivest, A. Shamir, and L.M. Adleman. A method for obtaining digital signatures and public-key cryptosystems. Communications of the $A C M$, (2):120-126, 1978.

[88] A. Rukhin, J. Soto, J. Nechvatal, M. Smid, E. Barker, S. Leigh, M. Levenson, M. Vangel, D. Banks, A. Heckert, J. Dray, and S. Vo. A statistical test suite for the validation of random number generators and pseudo random number generators for cryptographic applications. NIST, 2001. http://csrc.nist. gov/rng/rng2.html.

[89] J. Scharinger. Secure and fast encryption using chaotic Kolmogorov flows. Johannes Kepler University, Department of System Theory, 1998. http://www.cast.uni-linz.ac.at/Department/Publications/ Pubs1998/Scharinger98f.htm.

[90] B. Schneier. Applied cryptography, second edition. John Wiley \& Sons, Inc, 1996. ISBN 0-471-12845-7, http://www.ssl.stu.neva.ru/psw/crypto/ appl_rus/appl_cryp.htm.

[91] C. E. Shannon. A mathematical theory of communication. Bell System Technical Journal, 27(4):379-423, 623-526, 1948.

[92] C. E. Shannon. Communication theory of secrecy systems. Bell System Technical Journal, 28(4):656-715, 1949.

[93] M. I. Sobhy and A. E. D. Schehata. Secure e-mail and databases using chaotic encryption. Electronic Letters, 36(10), 2000.

[94] M. Svensson and J. E. Malmquist. A simple secure communications system utilizing chaotic functions to control the encryption and decryption of messages. Project report for the course 'Chaos in science and technology', 
Lund Institute of Technology, Dept. of physics, Subdept. of mathematical physics, 1996. http://www.efd.1th.se/ d92ms/chaoscrypt.html.

[95] J. Szczepański and Z. Kotulski. Pseudorandom number generators based on chaotic dynamical systems. Open Systems $\&$ Information Dynamics, 8(2):137146, 2001. http://www.ippt.gov.pl/ zkotulsk/open.pdf.

[96] A. Tsonis, editor. Chaos: from theory to applications. Plenum Press, New York, 1992.

[97] H. Waelbroeck and F. Zertuche. Discrete chaos, 1998. http://papaya. nuclecu. unam.mx/ nncp/chaos98.ps.

[98] J. Walker. A pseudorandom number sequence test program, 1998. http: //ww. fourmilab.ch/random/.

[99] D. D. Wheeler. Problems with chaotic cryptosystems. Cryptologia, (12):243$250,1989$.

[100] D. D. Wheeler. Supercomputer investigations of a chaotic encryption algorithm. Cryptologia, (15):140-150, 1991.

[101] H. White. Algorithmic complexity of points in dynamical systems. Ergodicity Theory Dynamical Systems, 13, 1993.

[102] S. Wolfram. Random sequence generation by cellular automata. Advances in Applied Mathematics, (7), 1986.

[103] W. K. Wong. Chaotic encryption technique. City University of Hong Kong, Department of Electronic Engineering, Hong Kong, 1999. http://kitson. netfirms.com/chaos/.

[104] C. J. Wu and Y. C. Lee. Observer-based method for secure communication of chaotic systems. Electronic Letters, 36(22), 1999.

[105] A. C. Yao. Theory of applications of trapdoor functions. In Proc. of IEEE Symp. on Foundations of Computer Science, pages 80-91, 1982. 
[106] Philip R. Zimmerman. The international PGP home page. http://www.pgpi. org/pgpi/. 


\section{Index}

\begin{tabular}{|c|c|}
\hline AES, 29 & asymmetric, 27 \\
\hline analytical solution, 75,82 & block, 29 \\
\hline attack, 34 & stream, 29 \\
\hline attractor, 37,43 & symmetric, 27 \\
\hline attractors, 37 & transposition, 28 \\
\hline \multirow{2}{*}{ bifurcation, 40} & Vernam, 31 \\
\hline & ciphertext, 26 \\
\hline cellular automata, 86 & class BPP, 49 \\
\hline chaos, 36 & class NP, 49 \\
\hline binary, 41,84 & class $P, 49$ \\
\hline continuos, 36 & complexity, 46,47 \\
\hline chaos theory, 19 & algorithmic, 50 \\
\hline chaotic map & symbolic, 51 \\
\hline Baker map, 87 & trajectory, 55 \\
\hline cellular automata, 86 & compromise, 33 \\
\hline Chebyshev map, 71 & confusion, 32 \\
\hline discrete tent map, 86 & cryptanalysis, 17,33 \\
\hline logistic map, 72 & cryptography, 15 \\
\hline Matthews map, 76 & cryptology, 17 \\
\hline multi-valued map, 82 & decrvption. 26 \\
\hline RANROT, 85 & $\begin{array}{l}\text { decryption, } 20 \\
\text { DFS } 29\end{array}$ \\
\hline sawteeth map, 62 & \\
\hline tent map, 76 & \\
\hline chaotic system & E-Larm, 91 \\
\hline binary, 41,84 & encryption, 26 \\
\hline real space, 36 & encryption scheme \\
\hline cipher, 26 & see cipher, 26 \\
\hline
\end{tabular}


entropy, 47

approximated, 109

conditional, 52

Kolmogorov-Sinai, 54

KS, 39

Shannon, 52

symbolic trajectory, 54

ergodicity, 40

fractal, 37

function

hash, 58

one-way, 57

Hamming distance, 41

hard-core predicate, 58

incompressibility, 46, 50

independence

asymptotic, 62

information, 24

information security, 15

initial condition, 25, 35

Kerchhoffs principle, 33

key, 26

Kolmogorov flows, 87

language, 49

level of significance, 104

LFSR, 85

Lyapunov exponents, 39

for binary pseudo-chaos, 85

Markov sequence, 53 one-time pad, 31

orbit, 25,35

p-value, 105

partitioning, 54, 92

LSB, 92

threshold, 93

PDF, 52

PGP, 27

plaintext, 24

PRNG, 32, 59

probability distribution function, 52

probability ensemble, 56

pseudo-chaos, 65

pseudo-random generator, 32,59

chaos-based, 61

multi-stream, 79

pseudo-randomness, 46, 57

randomness

algorithmic, 46, 47, 50

asymptotic, 62

tests, 102

true, 45,52

RSA, 27

session, 97

state space, 37

substitution, 28

symbolic dynamics, 54

synergetics, 17

system

chaotic, 36

cryptographic, 25 
dynamic, continuous, 35

ergodic, 40

measure preserving, 40

mixing, 41

trajectory, 25, 35

ideal, 67

symbolic, 54

transposition, 28

Turing machine, 48

deterministic, 49

nondeterministic, 49

probabilistic, 49

unpredictability

absolute, 45

computational, 46

white noise, 45 